\title{
9. GEOCHEMICAL EXPRESSION OF EARLY DIAGENESIS IN MIDDLE EOCENE-LOWER OLIGOCENE PELAGIC SEDIMENTS IN THE SOUTHERN LABRADOR SEA, SITE 647, ODP LEG 105
}

\author{
M. A. Arthur, ${ }^{2}$ W. E. Dean,${ }^{3}$ J. C. Zachos,${ }^{2}$ M. Kaminski, ${ }^{4}$ S. Hagerty Rieg, ${ }^{2}$ and K. Elmstrom ${ }^{2}$
}

\begin{abstract}
Geochemical analyses of the middle Eocene through lower Oligocene lithologic Unit IIIC (260-518 meters below seafloor [mbsf]) indicate a relatively constant geochemical composition of the detrital fraction throughout this depositional interval at Ocean Drilling Program (ODP) Site 647 in the southern Labrador Sea. The main variability occurs in redox-sensitive elements (e.g., iron, manganese, and phosphorus), which may be related to early diagenetic mobility in anaerobic pore waters during bacterial decomposition of organic matter. Initial preservation of organic matter was mediated by high sedimentation rates $(36 \mathrm{~m} / \mathrm{m}$.y.). High iron (Fe) and manganese $(\mathrm{Mn})$ contents are associated with carbonate concretions of siderite, manganosiderite, and rhodochrosite. These concretions probably formed in response to elevated pore-water alkalinity and total dissolved carbon dioxide $\left(\mathrm{CO}_{2}\right)$ concentrations resulting from bacterial sulfate reduction, as indicated by nodule stable-isotope compositions and pore-water geochemistry. These nodules differ from those found in upper Cenozoic hemipelagic sequences in that they are not associated with methanogenesis. Phosphate minerals (carbonate-fluorapatite) precipitated in some intervals, probably as the result of desorption of phosphorus from iron and manganese during reduction.

The bulk chemical composition of the sediments differs little from that of North Atlantic Quaternary abyssal red clays, but may contain a minor hydrothermal component. The silicon/ aluminum ( $\mathrm{Si} / \mathrm{Al})$ ratio, however, is high and variable and probably reflects original variations in biogenic opal, much of which is now altered to smectite and/or opal CT. An increase in the sodium/potassium $(\mathrm{Na} / \mathrm{K})$ ratio in the upper Eocene corresponds to the beginning of coarsergrained feldspar flux to the site, possibly marking the onset of more vigorous deep currents.

Although the Site 647 cores provide a nearly complete high-resolution, high-latitude Eocene-Oligocene record, the high sedimentation rate and somewhat unusual diagenetic conditions have led to variable alteration of benthic foraminifers and fine-fraction carbonate and have overprinted the original stable-isotope records. Planktonic foraminifers are less altered, but on the whole, there is little chance of sorting out the nature and timing of environmental change on the basis of our stable-isotope analyses.
\end{abstract}

\section{INTRODUCTION}

A total of 117 samples of predominantly grayish-green, middle Eocene through lower Oligocene, nannofossil claystone and clayey nannofossil chalk from lithologic Unit IIIC (260-518 mbsf; Cores 105-647A-28R through -54R) in Hole 647A were collected for inorganic geochemical and stable isotopic analyses. The samples were collected initially for the following purposes:

1. To document possible changes in the geochemical composition of sediments and stable isotopic compositions of calcareous biogenic components in response to changes in paleoclimatic and ocean circulation during the late Eocene to early Oligocene at high northern latitudes.

2. To document the composition of unusual authigenic nodules and/or beds in lithologic Unit IIIC.

The generally good core recovery, an apparently complete Eocene/Oligocene transition, and seemingly good (visual) preservation of calcareous microfossils led us to believe that our results would have a bearing on interpretation of climate and circulation

\footnotetext{
${ }^{1}$ Srivastava, S. P., Arthur, M., Clement, B., et al., 1989. Proc. ODP, Sci. Results, 105: College Station, TX (Ocean Drilling Program).

2 Graduate School of Oceanography, University of Rhode Island, Narragansett, RI 02882.

3 U.S. Geological Survey, Box 25046, MS 939, Federal Center, Denver, CO 80225 .

${ }^{4}$ Woods Hole Oceanographic Institution, Woods Hole, MA 02543. Now at Centre for Marine Geology, Dalhousie University, Halifax, Nova Scotia B3H, Canada.
}

events that occurred in latest Eocene to earliest Oligocene time. However, as shown below, the most significant application for our geochemical and isotopic data is in the documentation of a rather unusual early diagenetic regime.

\section{METHODS}

Samples were freeze-dried and ground to pass through a 100-mesh $(149 \mu \mathrm{m})$ sieve. Splits of all samples were analyzed coulometrically (Huffman, 1985) for carbonate and total carbon (precision better than $1 \%$ ) at the University of Rhode Island (URI). In addition, splits of many samples were sieved and selected for planktonic and benthic foraminifers for stable isotope studies. Splits of the ground samples were analyzed at the U.S. Geological Survey (USGS), Denver, for 10 major and minor elements by X-ray fluorescence (XRF) and 30 major, minor, and trace elements by induction-coupled, argon-plasma emission spectrometry (ICP) (Baedecker, 1987). Nine elements (aluminum, iron, magnesium, calcium, sodium, potassium, titanium, phosphorus, and manganese) were analyzed by both XRF and ICP, with essentially identical results between the two methods. Five samples contained concentrations of iron ( $\mathrm{Fe}$ ) and manganese $(\mathrm{Mn})$ that were sufficiently high to cause interferences with XRF; hence, no XRF results are available for these samples. Analytical results are given in Table 1 and plotted vs. depth in Figures 1 through 6 . The geochemical data have not been corrected for contribution of pore-water salts because porosities are typically lower than $40 \%$. Sodium $(\mathrm{Na})$ and magnesium $(\mathrm{Mg})$ concentrations would be the main elements affected.

Carbonate components were analyzed for carbon and oxygen-isotopic compositions at URI. Samples were ground to $<50 \mu \mathrm{m}$, roasted in vacuo at $390^{\circ} \mathrm{C}$ for $1 \mathrm{hr}$ and reacted online in purified phosphoric acid at $50^{\circ} \mathrm{C}$. The resulting $\mathrm{CO}_{2}$ gas was purified and introduced into a VG Micromass 602D mass spectrometer for analysis. Results are expressed in the standard delta notation relative to the PDB standard, where

$$
\delta=(\text { Rsamp-Rstd) } \times 1000 \text { Rstd, }
$$

and analytical precision was $0.1 \%$ for $\delta^{18} \mathrm{O}$ and $0.05 \%$ for $\delta^{13} \mathrm{C}$. 


\begin{tabular}{|c|c|c|c|c|c|c|c|c|c|c|c|c|c|c|c|c|c|}
\hline Sample & Core & Section & Interval & $\begin{array}{l}\text { Depth } \\
\text { (m) }\end{array}$ & $\begin{array}{c}\mathrm{SiO}_{2} \text {-xrf } \\
(\%)\end{array}$ & $\begin{array}{c}\mathrm{Al}_{2} \mathrm{O}_{3}-\mathrm{xrf} \\
(\%)\end{array}$ & $\begin{array}{c}\mathrm{Al}_{2} \mathrm{O}_{3} \text {-icp } \\
(\%)\end{array}$ & $\begin{array}{c}\mathrm{Fe}_{2} \mathrm{O}_{3}-\mathrm{xrf} \\
(\%)\end{array}$ & $\begin{array}{c}\mathrm{Fe}_{2} \mathrm{O}_{3} \text {-icp } \\
(\%)\end{array}$ & $\underset{(\%)}{\mathrm{MgO}-x \mathrm{rf}}$ & $\underset{(\%)}{\mathrm{MgO}-\mathrm{icp}}$ & $\begin{array}{c}\mathrm{CaO}-\mathrm{xrf} \\
(\%)\end{array}$ & $\begin{array}{c}\text { CaO-icp } \\
(\%)\end{array}$ & $\begin{array}{c}\mathrm{Na}_{2} \mathrm{O}-\mathrm{xrf} \\
(\%)\end{array}$ & $\begin{array}{c}\mathrm{Na}_{2} \mathrm{O} \text {-icp } \\
(\%)\end{array}$ & $\begin{array}{c}\mathrm{K}_{2} \mathrm{O}-\mathrm{xrf} \\
(\%)\end{array}$ & $\begin{array}{c}\mathrm{K}_{2} \mathrm{O} \text {-icp } \\
(\%)\end{array}$ \\
\hline 281089 & 28.00 & 1.00 & 89.00 & 260.99 & 40.80 & 10.40 & 10.96 & 4.88 & 4.86 & 1.93 & 1.99 & 18.60 & 16.80 & 1.51 & 1.62 & 1.55 & 1.80 \\
\hline 282052 & 28.00 & 2.00 & 52.00 & 262.12 & 36.70 & 8.57 & 9.26 & 5.69 & 5.72 & 1.78 & 1.83 & 21.20 & 19.60 & 1.35 & 1.62 & 1.43 & 1.92 \\
\hline 283054 & 28.00 & 3.00 & 54.00 & 263.65 & 28.80 & 7.79 & 8.50 & 3.32 & 3.29 & 1.50 & 1.56 & 28.90 & 26.60 & 1.24 & 1.48 & 0.49 & 1.20 \\
\hline 284068 & 28.00 & 4.00 & 68.00 & 265.28 & 49.60 & 11.80 & 12.47 & 4.84 & 4.72 & 2.14 & 2.16 & 11.90 & 11.34 & 1.84 & 1.89 & 1.62 & 1.68 \\
\hline 301051 & 30.00 & 1.00 & 51.00 & 279.93 & 26.60 & 7.51 & 8.12 & 6.93 & 6.86 & 1.85 & 1.83 & 23.40 & 22.40 & 1.16 & 1.31 & 0.62 & 1.19 \\
\hline 302092 & 30.00 & 2.00 & 92.00 & 281.82 & 33.20 & 9.10 & 9.82 & 3.71 & 3.72 & 1.59 & 1.61 & 24.60 & 23.80 & 1.42 & 1.62 & 0.99 & 1.44 \\
\hline 303045 & 30.00 & 3.00 & 45.00 & 282.85 & 29.00 & 7.94 & 8.69 & 3.03 & 3.00 & 1.43 & 1.46 & 29.20 & 28.00 & 1.24 & 1.35 & 0.61 & 1.32 \\
\hline 304098 & 30.00 & 4.00 & 98.00 & 284.88 & 36.30 & 9.84 & 10.39 & 3.81 & 3.58 & 1.66 & 1.66 & 23.10 & 22.40 & 1.40 & 1.48 & 1.19 & 1.56 \\
\hline 305112 & 30.00 & 5.00 & 112.00 & 286.52 & 38.50 & 10.50 & 11.15 & 4.09 & 3.86 & 1.82 & 1.83 & 20.80 & 19.60 & 1.48 & 1.48 & 1.35 & 1.56 \\
\hline 306097 & 30.00 & 6.00 & 97.00 & 287.87 & 36.10 & 9.92 & 10.58 & 3.83 & 3.58 & 1.67 & 1.66 & 22.50 & 21.00 & 1.32 & 1.48 & 1.13 & 1.44 \\
\hline 307029 & 30.00 & 7.00 & 29.00 & 288.69 & 42.90 & 11.90 & 12.66 & 4.39 & 4.29 & 2.00 & 1.99 & 16.50 & 15.40 & 1.55 & 1.62 & 1.67 & 1.92 \\
\hline 311029 & 31.00 & 1.00 & 29.00 & 289.29 & 39.20 & 10.50 & 11.33 & 4.30 & 4.29 & 1.79 & 1.83 & 20.30 & 19.60 & 1.40 & 1.62 & 1.25 & 1.56 \\
\hline 312102 & 31.00 & 2.00 & 102.00 & 291.51 & 37.70 & 9.28 & 10.01 & 5.20 & 5.15 & 1.77 & 1.83 & 20.00 & 18.20 & 1.29 & 1.48 & 1.20 & 1.32 \\
\hline 321049 & 32.00 & 1.00 & 49.00 & 299.09 & 36.00 & 7.97 & 8.69 & 3.43 & 3.43 & 1.45 & 1.49 & 25.20 & 23.80 & 1.31 & 1.48 & 0.96 & 1.20 \\
\hline 322032 & 32.00 & 2.00 & 32.00 & 300.42 & 43.50 & 7.75 & 8.31 & 3.35 & 3.43 & 1.37 & 1.39 & 20.90 & 19.60 & 1.26 & 1.35 & 1.05 & 1.14 \\
\hline 332018 & 33.00 & 2.00 & 18.00 & 309.98 & 36.00 & 8.88 & 9.45 & 4.11 & 4.15 & 1.59 & 1.64 & 23.40 & 22.40 & 1.31 & 1.48 & 0.97 & 1.32 \\
\hline 351028 & 35.00 & 1.00 & 28.00 & 327.88 & 44.40 & 11.40 & 12.09 & 4.57 & 4.29 & 1.87 & 1.83 & 16.20 & 15.40 & 1.48 & 1.48 & 1.65 & 1.80 \\
\hline 352029 & 35.00 & 2.00 & 29.00 & 329.39 & 43.90 & 11.30 & 11.90 & 4.58 & 4.29 & 1.83 & 1.83 & 17.10 & 16.80 & 1.39 & 1.48 & 1.55 & 1.68 \\
\hline 353024 & 35.00 & 3.00 & 24.00 & 332.63 & 43.90 & 11.30 & 11.90 & 4.33 & 4.15 & 1.82 & 1.83 & 17.00 & 16.80 & 1.38 & 1.48 & 1.65 & 1.80 \\
\hline 361068 & 36.00 & 1.00 & 68.00 & 337.88 & 43.00 & 10.70 & 11.15 & 4.67 & 4.43 & 1.80 & 1.83 & 17.10 & 16.80 & 1.40 & 1.62 & 1.53 & 1.68 \\
\hline 362068 & 36.00 & 2.00 & 68.00 & 339.38 & 45.10 & 11.20 & 12.09 & 5.00 & 5.01 & 1.84 & 1.83 & 15.40 & 14.00 & 1.39 & 1.48 & 1.59 & 1.80 \\
\hline 363068 & 36.00 & 3.00 & 68.00 & 340.88 & 42.90 & 10.40 & 11.15 & 6.74 & 6.72 & 1.71 & 1.66 & 15.00 & 14.00 & 1.24 & 1.35 & 1.59 & 1.68 \\
\hline 364041 & 36.00 & 4.00 & 42.00 & 342.13 & - & - & 3.02 & - & 31.46 & - & 3.98 & - & 9.24 & - & 0.57 & - & 0.42 \\
\hline 364068 & 36.00 & 4.00 & 68.00 & 342.38 & 44.20 & 11.10 & 11.52 & 4.70 & 4.58 & 1.82 & 1.83 & 16.10 & 15.40 & 1.37 & 1.48 & 1.60 & 1.68 \\
\hline 372014 & 37.00 & 2.00 & 15.00 & 348.35 & 42.90 & 10.50 & 11.15 & 4.32 & 4.29 & 1.74 & 1.83 & 17.90 & 16.80 & 1.35 & 1.48 & 1.46 & 1.56 \\
\hline 373015 & 37.00 & 3.00 & 15.00 & 349.85 & 40.70 & 10.30 & 10.96 & 4.18 & 4.15 & 1.66 & 1.66 & 19.70 & 18.20 & 1.21 & 1.29 & 1.41 & 1.56 \\
\hline 374015 & 37.00 & 4.00 & 15.00 & 351.35 & 40.10 & 9.36 & 10.01 & 4.18 & 4.00 & 1.54 & 1.58 & 20.90 & 19.60 & 1.23 & 1.35 & 1.29 & 1.56 \\
\hline 381036 & 38.00 & 1.00 & 36.00 & 356.76 & 38.00 & 8.72 & 9.26 & 4.02 & 3.86 & 1.43 & 1.44 & 23.10 & 22.40 & 1.24 & 1.29 & 1.13 & 1.44 \\
\hline 382040 & 38.00 & 2.00 & 39.00 & 358.30 & 43.60 & 10.20 & 10.77 & 4.35 & 4.15 & 1.64 & 1.63 & 18.10 & 16.80 & 1.31 & 1.35 & 1.48 & 1.56 \\
\hline 383041 & 38.00 & 3.00 & 41.00 & 359.81 & 44.30 & 10.00 & 10.58 & 4.31 & 4.29 & 1.57 & 1.63 & 17.90 & 16.80 & 1.34 & 1.35 & 1.49 & 1.68 \\
\hline 384041 & 38.00 & 4.00 & 41.00 & 361.31 & 39.40 & 8.90 & 9.45 & 4.01 & 4.00 & 1.47 & 1.49 & 21.70 & 21.00 & 1.08 & 1.31 & 1.18 & 1.44 \\
\hline 385041 & 38.00 & 5.00 & 41.00 & 362.81 & 47.40 & 10.80 & 11.52 & 4.77 & 4.72 & 1.74 & 1.83 & 14.90 & 14.00 & 1.33 & 1.48 & 1.58 & 1.68 \\
\hline 386066 & 38.00 & 6.00 & 67.00 & 364.56 & - & - & 2.27 & - & 31.46 & - & 3.98 & - & 9.66 & - & 0.57 & - & 0.32 \\
\hline 391017 & 39.00 & 1.00 & 17.00 & 366.27 & 53.90 & 12.10 & 12.47 & 5.08 & 5.01 & 1.92 & 1.99 & 9.57 & 9.24 & 1.54 & 1.62 & 1.83 & 1.92 \\
\hline 392017 & 39.00 & 2.00 & 17.00 & 367.77 & 46.60 & 10.50 & 11.15 & 4.96 & 4.86 & 1.67 & 1.66 & 15.70 & 15.40 & 1.29 & 1.35 & 1.60 & 1.68 \\
\hline 393019 & 39.00 & 3.00 & 19.00 & 369.29 & 45.80 & 10.30 & 10.96 & 4.37 & 4.29 & 1.65 & 1.66 & 16.10 & 15.40 & 1.33 & 1.35 & 1.52 & 1.68 \\
\hline 411030 & 41.00 & 1.00 & 30.00 & 385.70 & 34.70 & 8.66 & 9.07 & 10.50 & 9.87 & 2.07 & 2.16 & 16.40 & 15.40 & 1.14 & 1.17 & 1.20 & 1.44 \\
\hline 412030 & 41.00 & 2.00 & 30.00 & 387.20 & 37.10 & 9.44 & 10.01 & 3.80 & 3.58 & 1.45 & 1.46 & 23.60 & 22.40 & 1.14 & 1.19 & 1.22 & 1.44 \\
\hline 413030 & 41.00 & 3.00 & 30.00 & 388.70 & 36.20 & 9.34 & 9.82 & 3.77 & 3.58 & 1.42 & 1.43 & 24.00 & 22.40 & 1.20 & 1.24 & 1.25 & 1.44 \\
\hline 414030 & 41.00 & 4.00 & 30.00 & 390.20 & 40.60 & 10.60 & 11.33 & 4.21 & 4.15 & 1.60 & 1.64 & 20.00 & 19.60 & 1.25 & 1.35 & 1.66 & 1.92 \\
\hline 415030 & 41.00 & 5.00 & 30.00 & 391.70 & 27.50 & 7.12 & 7.74 & 13.40 & 13.44 & 2.03 & 2.16 & 17.30 & 16.80 & 0.97 & 1.06 & 1.11 & 1.44 \\
\hline 416070 & 41.00 & 6.00 & 70.00 & 393.60 & 21.60 & 6.10 & 6.61 & 16.60 & 17.16 & 2.27 & 2.32 & 17.20 & 16.80 & 0.82 & 0.96 & 0.59 & 1.09 \\
\hline 421042 & 42.00 & 1.00 & 42.00 & 395.52 & 37.50 & 10.50 & 11.33 & 3.77 & 3.72 & 1.52 & 1.53 & 22.00 & 21.00 & 1.13 & 1.24 & 1.63 & 1.80 \\
\hline 422048 & 42.00 & 2.00 & 48.00 & 397.07 & 34.70 & 9.81 & 10.39 & 4.33 & 4.29 & 1.41 & 1.41 & 23.40 & 22.40 & 1.08 & 1.16 & 1.55 & 1.92 \\
\hline 424014 & 42.00 & 4.00 & 14.00 & 399.74 & 33.60 & 9.38 & 10.20 & 4.87 & 4.86 & 1.37 & 1.39 & 24.20 & 22.40 & 1.02 & 1.15 & 1.54 & 1.92 \\
\hline 425028 & 42.00 & 5.00 & 28.00 & 401.38 & 27.60 & 7.61 & 8.31 & 4.64 & 4.72 & 1.20 & 1.21 & 29.30 & 28.00 & 0.96 & 1.05 & 1.06 & 1.68 \\
\hline 426047 & 42.00 & 6.00 & 47.00 & 403.07 & 30.90 & 8.80 & 9.45 & 3.76 & 3.86 & 1.27 & 1.29 & 27.30 & 25.20 & 0.97 & 1.06 & 1.15 & 1.68 \\
\hline 427049 & 42.00 & 7.00 & 49.00 & 404.59 & 31.90 & 8.78 & 9.45 & 4.14 & 4.00 & 1.28 & 1.33 & 26.40 & 25.20 & 1.05 & 1.13 & 1.25 & 1.80 \\
\hline 431086 & 43.00 & 1.00 & 86.00 & 405.66 & 36.00 & 9.87 & 10.39 & 4.79 & 4.58 & 1.50 & 1.51 & 22.70 & 21.00 & 1.32 & 1.29 & 1.50 & 1.92 \\
\hline 432029 & 43.00 & 2.00 & 29.00 & 406.59 & 38.20 & 10.70 & 11.33 & 4.29 & 4.00 & 1.56 & 1.54 & 21.00 & 19.60 & 1.25 & 1.24 & 1.59 & 1.80 \\
\hline 433083 & 43.00 & 3.00 & 83.00 & 408.63 & 43.80 & 11.20 & 11.90 & 7.01 & 6.86 & 1.79 & 1.83 & 14.50 & 13.58 & 1.30 & 1.33 & 2.13 & 2.28 \\
\hline 434025 & 43.00 & 4.00 & 25.00 & 409.55 & 21.20 & 5.59 & 6.23 & 4.31 & 4.43 & 1.16 & 1.21 & 31.30 & 29.40 & 1.17 & 1.35 & 0.81 & 1.06 \\
\hline 435018 & 43.00 & 5.00 & 18.00 & 410.98 & 34.30 & 8.95 & 9.63 & 5.58 & 5.58 & 1.60 & 1.61 & 22.10 & 21.00 & 1.05 & 1.19 & 1.29 & 1.44 \\
\hline 436027 & 43.00 & 6.00 & 27.00 & 412.57 & 47.40 & 12.40 & 12.85 & 6.52 & 6.43 & 1.94 & 1.99 & 12.10 & 11.62 & 1.34 & 1.35 & 2.22 & 2.40 \\
\hline 437026 & 43.00 & 7.00 & 26.00 & 414.06 & 46.60 & 12.60 & 13.22 & 4.76 & 4.72 & 1.84 & 1.83 & 13.30 & 12.74 & 1.34 & 1.35 & 1.96 & 2.04 \\
\hline 441080 & 44.00 & 1.00 & 80.00 & 415.30 & 41.10 & 10.90 & 11.71 & 4.73 & 4.72 & 1.66 & 1.66 & 18.70 & 18.20 & 1.28 & 1.35 & 1.55 & 1.80 \\
\hline 442082 & 44.00 & 2.00 & 82.00 & 416.82 & 43.00 & 11.50 & 12.47 & 4.45 & 4.29 & 1.67 & 1.83 & 17.50 & 16.80 & 1.32 & 1.35 & 1.74 & 1.92 \\
\hline
\end{tabular}




\begin{tabular}{|c|c|c|c|c|c|c|c|c|c|c|c|c|c|c|c|c|c|}
\hline 443087 & 44.00 & 3.00 & 87.00 & 418.36 & 38.20 & 10.60 & 11.15 & 4.02 & 3.72 & 1.56 & 1.56 & 21.80 & 19.60 & 1.24 & 1.20 & 1.67 & 1.92 \\
\hline 444116 & 44.00 & 4.00 & 116.00 & 420.16 & 33.50 & 9.33 & 10.01 & 3.43 & 3.43 & 1.31 & 1.36 & 25.60 & 23.80 & 1.03 & 1.11 & 1.33 & 1.80 \\
\hline 445074 & 44.00 & 5.00 & 74.00 & 421.24 & 29.10 & 8.06 & 8.69 & 3.00 & 2.86 & 1.17 & 1.16 & 29.90 & 28.00 & 0.97 & 1.04 & 0.72 & 1.44 \\
\hline 446064 & 44.00 & 6.00 & 64.00 & 422.64 & 33.90 & 9.23 & 10.01 & 3.52 & 3.58 & 1.34 & 1.36 & 25.40 & 23.80 & 1.06 & 1.13 & 1.06 & 1.44 \\
\hline 451102 & 45.00 & 1.00 & 102.00 & 425.11 & 39.80 & 10.20 & 10.96 & 4.39 & 4.29 & 1.55 & 1.56 & 20.50 & 19.60 & 1.15 & 1.24 & 1.56 & 1.68 \\
\hline 453055 & 45.00 & 3.00 & 55.00 & 427.65 & 38.50 & 9.63 & 10.39 & 4.04 & 4.00 & 1.45 & 1.46 & 22.30 & 21.00 & 0.96 & 1.12 & 1.28 & 1.68 \\
\hline 461047 & 46.00 & 1.00 & 47.00 & 434.27 & 47.70 & 11.10 & 11.71 & 5.35 & 5.29 & 1.76 & 1.83 & 14.00 & 13.30 & 1.27 & 1.35 & 1.59 & 1.68 \\
\hline 462041 & 46.00 & 2.00 & 41.00 & 435.71 & 42.70 & 10.20 & 10.96 & 4.26 & 4.29 & 1.57 & 1.64 & 19.10 & 18.20 & 1.28 & 1.35 & 1.44 & 1.56 \\
\hline 463041 & 46.00 & 3.00 & 41.00 & 437.21 & 46.40 & 11.50 & 11.90 & 5.61 & 5.29 & 1.78 & 1.83 & 13.80 & 13.16 & 1.36 & 1.32 & 1.68 & 1.80 \\
\hline 464041 & 46.00 & 4.00 & 41.00 & 438.71 & 52.40 & 13.00 & 13.60 & 5.40 & 5.01 & 1.95 & 1.99 & 9.94 & 9.52 & 1.42 & 1.48 & 1.85 & 1.92 \\
\hline 465057 & 46.00 & 5.00 & 57.00 & 440.37 & 47.00 & 11.80 & 12.28 & 6.48 & 6.01 & 1.89 & 1.83 & 13.30 & 12.74 & 1.31 & 1.48 & 1.74 & 1.92 \\
\hline 465111 & 46.00 & 5.00 & 111.00 & 440.91 & 29.40 & 7.52 & 8.31 & 3.46 & 3.29 & 1.27 & 1.26 & 28.10 & 26.60 & 1.40 & 1.62 & 0.91 & 1.12 \\
\hline 466057 & 46.00 & 6.00 & 57.00 & 441.87 & 46.50 & 11.70 & 12.28 & 5.29 & 5.15 & 1.76 & 1.83 & 14.20 & 13.30 & 1.37 & 1.35 & 1.65 & 1.80 \\
\hline 471033 & 47.00 & 1.00 & 33.00 & 443.82 & 46.70 & 11.20 & 11.90 & 7.27 & 7.29 & 1.88 & 1.99 & 13.10 & 12.46 & 1.30 & 1.35 & 1.98 & 2.16 \\
\hline 473016 & 47.00 & 3.00 & 16.00 & 446.66 & 49.00 & 12.50 & 13.03 & 5.30 & 5.15 & 1.88 & 1.83 & 12.20 & 11.48 & 1.32 & 1.35 & 1.75 & 1.80 \\
\hline 474021 & 47.00 & 4.00 & 21.00 & 448.21 & 45.00 & 11.20 & 11.90 & 7.16 & 7.01 & 1.85 & 1.83 & 13.70 & 13.02 & 1.29 & 1.33 & 1.57 & 1.68 \\
\hline 475021 & 47.00 & 5.00 & 21.00 & 449.71 & 39.10 & 9.56 & 10.20 & 4.61 & 4.58 & 1.52 & 1.54 & 21.20 & 19.60 & 1.18 & 1.25 & 1.41 & 1.56 \\
\hline 476025 & 47.00 & 6.00 & 25.00 & 451.25 & 59.00 & 14.30 & 13.60 & 9.30 & 8.87 & 2.30 & 2.16 & 0.97 & 0.94 & 1.33 & 1.27 & 3.39 & 3.24 \\
\hline 481007 & 48.00 & 1.00 & 7.00 & 453.27 & - & 3.21 & 3.21 & - & 37.18 & - & 3.65 & - & 6.16 & - & 0.39 & - & 0.58 \\
\hline 481030 & 48.00 & 1.00 & 30.00 & 453.50 & 50.00 & 11.90 & 12.47 & 9.20 & 8.72 & 2.11 & 2.16 & 9.24 & 8.96 & 1.51 & 1.48 & 2.38 & 2.52 \\
\hline 482034 & 48.00 & 2.00 & 34.00 & 455.04 & 40.80 & 9.97 & 10.58 & 4.36 & 4.15 & 1.62 & 1.61 & 20.40 & 19.60 & 1.29 & 1.28 & 1.32 & 1.44 \\
\hline 483029 & 48.00 & 3.00 & 29.00 & 456.49 & 52.80 & 11.30 & 11.71 & 6.09 & 5.72 & 1.88 & 1.83 & 10.60 & 10.22 & 1.34 & 1.35 & 1.71 & 1.80 \\
\hline 484032 & 48.00 & 4.00 & 32.00 & 458.02 & 48.90 & 11.30 & 12.09 & 4.68 & 4.58 & 1.79 & 1.83 & 13.70 & 12.88 & 1.15 & 1.28 & 1.53 & 1.68 \\
\hline 485030 & 48.00 & 5.00 & 30.00 & 459.50 & 58.30 & 12.50 & 12.85 & 5.70 & 5.43 & 2.03 & 1.99 & 6.34 & 6.02 & 1.43 & 1.35 & 1.76 & 1.80 \\
\hline 491074 & 49.00 & 1.00 & 74.00 & 463.54 & 47.40 & 11.40 & 12.09 & 4.66 & 4.58 & 1.94 & 1.99 & 14.20 & 13.44 & 1.31 & 1.48 & 1.55 & 1.68 \\
\hline 492066 & 49.00 & 2.00 & 66.00 & 464.96 & 48.20 & 11.30 & 11.90 & 4.71 & 4.72 & 1.87 & 1.83 & 13.80 & 13.30 & 1.34 & 1.35 & 1.52 & 1.68 \\
\hline 493080 & 49.00 & 3.00 & 80.00 & 466.60 & 33.40 & 7.80 & 8.31 & 8.23 & 8.01 & 2.09 & 2.16 & 17.30 & 16.80 & 1.07 & 1.23 & 0.95 & 1.20 \\
\hline 494039 & 49.00 & 4.00 & 39.00 & 467.69 & 37.60 & 8.81 & 9.45 & 5.81 & 5.72 & 1.75 & 1.83 & 18.80 & 18.20 & 1.16 & 1.28 & 1.27 & 1.44 \\
\hline 496043 & 49.00 & 6.00 & 43.00 & 470.73 & 40.60 & 7.97 & 8.69 & 3.48 & 3.43 & 1.36 & 1.43 & 22.60 & 22.40 & 1.16 & 1.16 & 1.05 & 1.20 \\
\hline 501056 & 50.00 & 1.00 & 56.00 & 473.06 & 43.70 & 10.10 & 10.58 & 4.75 & 4.43 & 1.80 & 1.83 & 17.10 & 16.80 & 1.19 & 1.29 & 1.44 & 1.56 \\
\hline 502056 & 50.00 & 2.00 & 56.00 & 474.56 & 45.20 & 10.50 & 11.15 & 5.29 & 4.86 & 1.84 & 1.83 & 15.60 & 15.40 & 1.09 & 1.31 & 1.60 & 1.68 \\
\hline 503036 & 50.00 & 3.00 & 36.00 & 475.86 & 46.30 & 11.00 & 11.52 & 4.59 & 4.29 & 1.82 & 1.83 & 15.60 & 15.40 & 1.24 & 1.33 & 1.53 & 1.56 \\
\hline 504032 & 50.00 & 4.00 & 32.00 & 477.32 & 29.40 & 6.46 & 6.99 & 9.50 & 9.58 & 2.02 & 2.16 & 17.60 & 16.80 & 0.89 & 1.13 & 0.77 & 1.09 \\
\hline 505033 & 50.00 & 5.00 & 33.00 & 478.83 & 62.60 & 13.00 & 13.22 & 6.80 & 6.72 & 2.07 & 1.99 & 2.55 & 2.52 & 1.37 & 1.35 & 2.48 & 2.52 \\
\hline 506012 & 50.00 & 6.00 & 12.00 & 480.12 & 30.70 & 6.88 & 7.37 & 10.60 & 10.58 & 2.14 & 2.16 & 14.60 & 13.86 & 0.87 & 1.02 & 1.08 & 1.20 \\
\hline 511031 & 51.00 & 1.00 & 31.00 & 482.40 & 46.30 & 10.70 & 11.33 & 4.95 & 4.86 & 1.80 & 1.83 & 15.80 & 15.40 & 1.26 & 1.28 & 1.64 & 1.68 \\
\hline 512036 & 51.00 & 2.00 & 36.00 & 483.96 & 38.00 & 8.85 & 9.45 & 4.17 & 4.15 & 1.58 & 1.58 & 21.30 & 19.60 & 1.06 & 1.13 & 1.15 & 1.32 \\
\hline 513035 & 51.00 & 3.00 & 35.00 & 485.45 & 39.30 & 8.96 & 9.63 & 4.16 & 4.00 & 1.55 & 1.59 & 21.50 & 21.00 & 1.11 & 1.16 & 1.23 & 1.44 \\
\hline 514068 & 51.00 & 4.00 & 68.00 & 487.28 & 4.84 & 1.10 & 1.25 & 16.40 & 17.16 & 2.47 & 2.66 & 18.90 & 18.20 & 0.33 & 0.65 & 0.07 & 0.17 \\
\hline 515024 & 51.00 & 5.00 & 24.00 & 488.34 & 43.50 & 10.20 & 10.77 & 6.60 & 6.15 & 2.00 & 1.99 & 14.20 & 13.44 & 1.28 & 1.28 & 1.61 & 1.68 \\
\hline 516083 & 51.00 & 6.00 & 83.00 & 490.43 & - & - & 3.40 & - & 18.59 & - & 3.15 & - & 12.60 & - & 0.65 & - & 0.48 \\
\hline 522010 & 52.00 & 2.00 & 10.00 & 493.40 & 31.30 & 7.09 & 7.56 & 8.38 & 7.86 & 1.99 & 1.99 & 19.70 & 18.20 & 0.92 & 1.06 & 0.92 & 1.10 \\
\hline 523098 & 52.00 & 3.00 & 98.00 & 495.78 & 38.70 & 6.89 & 7.56 & 5.74 & 5.86 & 1.67 & 1.66 & 19.80 & 19.60 & 1.06 & 1.15 & 0.97 & 1.16 \\
\hline 524056 & 52.00 & 4.00 & 56.00 & 496.86 & 38.20 & 8.34 & 9.07 & 3.85 & 3.86 & 1.40 & 1.48 & 23.50 & 22.40 & 1.03 & 1.12 & 1.18 & 1.44 \\
\hline 525069 & 52.00 & 5.00 & 69.00 & 498.49 & 36.80 & 9.24 & 10.01 & 5.22 & 5.15 & 1.68 & 1.66 & 21.20 & 19.60 & 0.94 & 1.11 & 1.31 & 1.56 \\
\hline 526076 & 52.00 & 6.00 & 76.00 & 500.06 & 59.20 & 14.00 & 14.36 & 9.81 & 9.87 & 2.45 & 2.32 & 1.34 & 1.36 & 1.24 & 1.32 & 3.23 & 3.24 \\
\hline 531047 & 53.00 & 1.00 & 47.00 & 501.87 & 44.90 & 10.90 & 11.52 & 5.10 & 5.01 & 1.80 & 1.83 & 16.20 & 15.40 & 1.22 & 1.27 & 1.66 & 1.80 \\
\hline 532008 & 53.00 & 2.00 & 8.00 & 502.98 & 60.00 & 14.10 & 14.36 & 8.79 & 9.01 & 2.27 & 2.32 & 0.77 & 0.80 & 1.33 & 1.29 & 2.72 & 2.76 \\
\hline 533055 & 53.00 & 3.00 & 55.00 & 504.95 & 47.20 & 10.20 & 10.77 & 5.52 & 5.29 & 1.73 & 1.83 & 14.90 & 14.00 & 1.22 & 1.23 & 1.67 & 1.80 \\
\hline 534079 & 53.00 & 4.00 & 79.00 & 506.45 & - & - & 2.27 & - & 17.16 & - & 2.66 & - & 15.40 & - & 0.81 & - & 0.32 \\
\hline 535018 & 53.00 & 5.00 & 18.00 & 507.58 & 42.30 & 9.90 & 10.39 & 5.41 & 5.15 & 1.67 & 1.66 & 18.50 & 18.20 & 1.10 & 1.15 & 1.55 & 1.68 \\
\hline 541025 & 54.00 & 1.00 & 25.00 & 511.35 & 38.10 & 9.45 & 10.01 & 8.89 & 8.44 & 2.06 & 2.16 & 15.40 & 14.00 & 1.00 & 1.17 & 1.39 & 1.44 \\
\hline 542013 & 54.00 & 2.00 & 13.00 & 512.73 & 40.30 & 9.04 & 9.82 & 5.43 & 5.43 & 1.69 & 1.83 & 19.00 & 18.20 & 1.09 & 1.19 & 1.29 & 1.44 \\
\hline 543016 & 54.00 & 3.00 & 16.00 & 514.26 & 49.00 & 12.40 & 13.03 & 6.30 & 6.15 & 1.98 & 1.99 & 11.50 & 10.92 & 1.25 & 1.32 & 2.01 & 2.16 \\
\hline 544123 & 54.00 & 4.00 & 123.00 & 516.83 & 60.40 & 13.00 & 13.41 & 9.63 & 9.72 & 2.18 & 2.16 & 2.60 & 2.66 & 1.14 & 1.19 & 2.72 & 2.88 \\
\hline 545011 & 54.00 & 5.00 & 11.00 & 517.20 & 50.30 & 11.10 & 11.71 & 4.90 & 4.86 & 1.81 & 1.83 & 12.60 & 12.04 & 1.21 & 1.24 & 1.61 & 1.68 \\
\hline 546014 & 54.00 & 6.00 & 14.00 & 518.74 & 40.40 & 8.19 & 8.69 & 3.61 & 3.72 & 1.33 & 1.39 & 22.40 & 21.00 & 1.03 & 1.06 & 1.08 & 1.20 \\
\hline
\end{tabular}




\begin{tabular}{|c|c|c|c|c|c|c|c|c|c|c|c|c|c|c|c|c|c|c|}
\hline Sample & Core & Section & Interval & $\begin{array}{c}\text { Depth } \\
\text { (m) }\end{array}$ & $\begin{array}{c}\mathrm{TiO}_{2} \text {-xrf } \\
(\%)\end{array}$ & $\begin{array}{c}\mathrm{TiO}_{2} \text {-icp } \\
(\%)\end{array}$ & $\begin{array}{c}\mathrm{P}_{2} \mathrm{O}_{5} \text {-xrf } \\
(\%)\end{array}$ & $\begin{array}{c}\mathrm{P}_{2} \mathrm{O}_{5} \text {-icp } \\
(\%)\end{array}$ & $\underset{(\%)}{\mathrm{MnO}-\mathrm{xrf}}$ & $\begin{array}{c}\text { MnO-icp } \\
(\%)\end{array}$ & $\begin{array}{c}\text { LOI } 900^{\circ} \mathrm{C} \\
(\%)\end{array}$ & $\underset{(\mathrm{ppm})}{\mathrm{Ba}}$ & $\begin{array}{c}\mathrm{Ce} \\
\text { (ppm) }\end{array}$ & $\begin{array}{c}\text { Co } \\
(\mathrm{ppm})\end{array}$ & $\begin{array}{c}\mathrm{Cr} \\
\text { (ppm) }\end{array}$ & $\underset{(\mathrm{ppm})}{\mathrm{Cu}}$ & $\underset{(\mathrm{ppm})}{\mathrm{Ga}}$ & $\underset{(\mathrm{ppm})}{\mathrm{La}}$ \\
\hline 281089 & 28.00 & 1.00 & 89.00 & 260.99 & 0.47 & 0.40 & 0.10 & 0.09 & 0.08 & 0.08 & 19.50 & 830.00 & 58.00 & 20.00 & 72.00 & 56.00 & 14.00 & 26.00 \\
\hline 282052 & 28.00 & 2.00 & 52.00 & 262.12 & 0.34 & 0.30 & 0.08 & 0.09 & 0.22 & 0.22 & 21.80 & 930.00 & 38.00 & 44.00 & 58.00 & 42.00 & 13.00 & 19.00 \\
\hline 283054 & 28.00 & 3.00 & 54.00 & 263.65 & 0.31 & 0.28 & 0.09 & 0.09 & 0.13 & 0.14 & 26.90 & 640.00 & 44.00 & 16.00 & 51.00 & 47.00 & 11.00 & 19.00 \\
\hline 284068 & 28.00 & 4.00 & 68.00 & 265.28 & 0.53 & 0.45 & 0.08 & 0.09 & 0.41 & 0.37 & 14.50 & 620.00 & 67.00 & 23.00 & 75.00 & 73.00 & 18.00 & 29.00 \\
\hline 301051 & 30.00 & 1.00 & 51.00 & 279.93 & 0.31 & 0.27 & 0.11 & 0.11 & 4.60 & 4.39 & 27.00 & 450.00 & 46.00 & 15.00 & 46.00 & 45.00 & 30.00 & 21.00 \\
\hline 302092 & 30.00 & 2.00 & 92.00 & 281.82 & 0.39 & 0.33 & 0.08 & 0.09 & 0.12 & 0.12 & 23.80 & 600.00 & 48.00 & 74.00 & 62.00 & 64.00 & 12.00 & 22.00 \\
\hline 303045 & 30.00 & 3.00 & 45.00 & 282.85 & 0.33 & 0.32 & 0.11 & 0.11 & 0.17 & 0.18 & 27.00 & 520.00 & 44.00 & 23.00 & 53.00 & 54.00 & 11.00 & 21.00 \\
\hline 304098 & 30.00 & 4.00 & 98.00 & 284.88 & 0.42 & 0.35 & 0.19 & 0.18 & 0.15 & 0.13 & 22.40 & 550.00 & 63.00 & 13.00 & 52.00 & 58.00 & 13.00 & 28.00 \\
\hline 305112 & 30.00 & 5.00 & 112.00 & 286.52 & 0.47 & 0.40 & 0.16 & 0.16 & 0.14 & 0.13 & 20.60 & 530.00 & 55.00 & 81.00 & 60.00 & 60.00 & 13.00 & 25.00 \\
\hline 306097 & 30.00 & 6.00 & 97.00 & 287.87 & 0.45 & 0.38 & 0.12 & 0.11 & 0.20 & 0.19 & 22.30 & 550.00 & 50.00 & 34.00 & 60.00 & 70.00 & 13.00 & 23.00 \\
\hline 307029 & 30.00 & 7.00 & 29.00 & 288.69 & 0.54 & 0.43 & 0.09 & 0.09 & 0.07 & 0.08 & 18.10 & 740.00 & 64.00 & 74.00 & 79.00 & 74.00 & 16.00 & 26.00 \\
\hline 311029 & 31.00 & 1.00 & 29.00 & 289.29 & 0.46 & 0.42 & 0.13 & 0.11 & 0.12 & 0.12 & 20.30 & 750.00 & 58.00 & 22.00 & 65.00 & 50.00 & 15.00 & 24.00 \\
\hline 312102 & 31.00 & 2.00 & 102.00 & 291.51 & 0.42 & 0.38 & 0.13 & 0.11 & 1.55 & 1.29 & 21.30 & 640.00 & 55.00 & 25.00 & 50.00 & 70.00 & 21.00 & 23.00 \\
\hline 321049 & 32.00 & 1.00 & 49.00 & 299.09 & 0.35 & 0.32 & 0.12 & 0.11 & 0.21 & 0.21 & 23.00 & 560.00 & 43.00 & 13.00 & 53.00 & 72.00 & 12.00 & 20.00 \\
\hline 322032 & 32.00 & 2.00 & 32.00 & 300.42 & 0.34 & 0.30 & 0.13 & 0.14 & 0.14 & 0.14 & 19.10 & 610.00 & 47.00 & 20.00 & 51.00 & 60.00 & 10.00 & 20.00 \\
\hline 332018 & 33.00 & 2.00 & 18.00 & 309.98 & 0.38 & 0.33 & 0.09 & 0.09 & 0.13 & 0.14 & 22.20 & 690.00 & 44.00 & 150.00 & 59.00 & 63.00 & 12.00 & 19.00 \\
\hline 351028 & 35.00 & 1.00 & 28.00 & 327.88 & 0.49 & 0.38 & 0.08 & 0.07 & 0.08 & 0.08 & 17.70 & 650.00 & 55.00 & 33.00 & 65.00 & 54.00 & 14.00 & 25.00 \\
\hline 352029 & 35.00 & 2.00 & 29.00 & 329.39 & 0.49 & 0.43 & 0.10 & 0.09 & 0.10 & 0.10 & 17.60 & 550.00 & 56.00 & 17.00 & 60.00 & 85.00 & 14.00 & 24.00 \\
\hline 353024 & 35.00 & 3.00 & 24.00 & 332.63 & 0.47 & 0.40 & 0.09 & 0.09 & 0.07 & 0.08 & 17.80 & 650.00 & 53.00 & 19.00 & 59.00 & 60.00 & 14.00 & 24.00 \\
\hline 361068 & 36.00 & 1.00 & 68.00 & 337.88 & 0.48 & 0.38 & 0.16 & 0.14 & 0.28 & 0.26 & 18.50 & 610.00 & 66.00 & 38.00 & 67.00 & 59.00 & 14.00 & 27.00 \\
\hline 362068 & 36.00 & 2.00 & 68.00 & 339.38 & 0.46 & 0.42 & 0.08 & 0.09 & 0.17 & 0.07 & 16.90 & 820.00 & 62.00 & 30.00 & 74.00 & 70.00 & 15.00 & 27.00 \\
\hline 363068 & 36.00 & 3.00 & 68.00 & 340.88 & 0.45 & 0.38 & 0.12 & 0.11 & 0.07 & 0.08 & 15.20 & 250.00 & 55.00 & 49.00 & 70.00 & 65.00 & 15.00 & 24.00 \\
\hline 364041 & 36.00 & 4.00 & 42.00 & 342.13 & - & 0.12 & - & 0.21 & - & 11.48 & - & 190.00 & 54.00 & 19.00 & 20.00 & 12.00 & - & 24.00 \\
\hline 364068 & 36.00 & 4.00 & 68.00 & 342.38 & 0.48 & 0.42 & 0.16 & 0.16 & 0.19 & 0.18 & 17.40 & 830.00 & 62.00 & 24.00 & 73.00 & 75.00 & 15.00 & 27.00 \\
\hline 372014 & 37.00 & 2.00 & 15.00 & 348.35 & 0.45 & 0.38 & 0.09 & 0.09 & 0.07 & 0.08 & 18.60 & 610.00 & 50.00 & 31.00 & 67.00 & 80.00 & 14.00 & 22.00 \\
\hline 373015 & 37.00 & 3.00 & 15.00 & 349.85 & 0.44 & 0.38 & 0.11 & 0.09 & 0.09 & 0.10 & 19.40 & 710.00 & 48.00 & 52.00 & 67.00 & 62.00 & 14.00 & 22.00 \\
\hline 374015 & 37.00 & 4.00 & 15.00 & 351.35 & 0.38 & 0.33 & 0.06 & 0.07 & 0.10 & 0.10 & 20.50 & 510.00 & 44.00 & 16.00 & 59.00 & 56.00 & 12.00 & 19.00 \\
\hline 381036 & 38.00 & 1.00 & 36.00 & 356.76 & 0.38 & 0.32 & 0.09 & 0.09 & 0.13 & 0.13 & 21.80 & 560.00 & 44.00 & 25.00 & 58.00 & 58.00 & 11.00 & 19.00 \\
\hline 382040 & 38.00 & 2.00 & 39.00 & 358.30 & 0.41 & 0.35 & 0.08 & 0.07 & 0.07 & 0.08 & 18.40 & 590.00 & 51.00 & 19.00 & 66.00 & 70.00 & 12.00 & 22.00 \\
\hline 383041 & 38.00 & 3.00 & 41.00 & 359.81 & 0.41 & 0.37 & 0.09 & 0.07 & 0.10 & 0.10 & 18.40 & 610.00 & 45.00 & 47.00 & 67.00 & 55.00 & 14.00 & 20.00 \\
\hline 384041 & 38.00 & 4.00 & 41.00 & 361.31 & 0.36 & 0.32 & 0.09 & 0.09 & 0.15 & 0.15 & 21.50 & 620.00 & 41.00 & 43.00 & 58.00 & 53.00 & 12.00 & 18.00 \\
\hline 385041 & 38.00 & 5.00 & 41.00 & 362.81 & 0.47 & 0.42 & 0.10 & 0.09 & 0.07 & 0.08 & 16.20 & 710.00 & 58.00 & 19.00 & 73.00 & 69.00 & 15.00 & 24.00 \\
\hline 386066 & 38.00 & 6.00 & 67.00 & 364.56 & - & 0.08 & - & 1.56 & - & 12.90 & - & 160.00 & 51.00 & 35.00 & 16.00 & 11.00 & - & 31.00 \\
\hline 391017 & 39.00 & 1.00 & 17.00 & 366.27 & 0.50 & 0.43 & 0.17 & 0.16 & 0.05 & 0.05 & 12.30 & 870.00 & 79.00 & 35.00 & 78.00 & 120.00 & 15.00 & 33.00 \\
\hline 392017 & 39.00 & 2.00 & 17.00 & 367.77 & 0.44 & 0.38 & 0.13 & 0.11 & 0.11 & 0.11 & 15.90 & 770.00 & 59.00 & 22.00 & 68.00 & 73.00 & 14.00 & 25.00 \\
\hline 393019 & 39.00 & 3.00 & 19.00 & 369.29 & 0.41 & 0.35 & 0.09 & 0.07 & 0.06 & 0.07 & 17.00 & 690.00 & 59.00 & 39.00 & 66.00 & 98.00 & 14.00 & 25.00 \\
\hline 411030 & 41.00 & 1.00 & 30.00 & 385.70 & 0.35 & 0.30 & 0.12 & 0.11 & 3.34 & 3.35 & 21.50 & 630.00 & 55.00 & 14.00 & 51.00 & 49.00 & 19.00 & 24.00 \\
\hline 412030 & 41.00 & 2.00 & 30.00 & 387.20 & 0.38 & 0.32 & 0.07 & 0.07 & 0.11 & 0.11 & 22.10 & 600.00 & 43.00 & 19.00 & 60.00 & 51.00 & 12.00 & 20.00 \\
\hline 413030 & 41.00 & 3.00 & 30.00 & 388.70 & 0.39 & 0.32 & 0.11 & 0.11 & 0.13 & 0.13 & 22.20 & 560.00 & 53.00 & 15.00 & 58.00 & 79.00 & 12.00 & 23.00 \\
\hline 414030 & 41.00 & 4.00 & 30.00 & 390.20 & 0.41 & 0.35 & 0.08 & 0.07 & 0.07 & 0.08 & 20.10 & 710.00 & 56.00 & 61.00 & 70.00 & 68.00 & 14.00 & 25.00 \\
\hline 415030 & 41.00 & 5.00 & 30.00 & 391.70 & 0.28 & 0.25 & 0.17 & 0.16 & 5.60 & 5.55 & 24.50 & 540.00 & 58.00 & 71.00 & 39.00 & 57.00 & - & 24.00 \\
\hline 416070 & 41.00 & 6.00 & 70.00 & 393.60 & 0.24 & 0.22 & 0.21 & 0.21 & 6.95 & 6.58 & 26.80 & 330.00 & 65.00 & 20.00 & 37.00 & 32.00 & - & 28.00 \\
\hline 421042 & 42.00 & 1.00 & 42.00 & 395.52 & 0.42 & 0.37 & 0.07 & 0.07 & 0.09 & 0.10 & 21.80 & 650.00 & 50.00 & 19.00 & 66.00 & 69.00 & 15.00 & 23.00 \\
\hline 422048 & 42.00 & 2.00 & 48.00 & 397.07 & 0.37 & 0.33 & 0.11 & 0.11 & 0.34 & 0.31 & 22.80 & 780.00 & 55.00 & 23.00 & 61.00 & 31.00 & 15.00 & 24.00 \\
\hline 424014 & 42.00 & 4.00 & 14.00 & 399.74 & 0.36 & 0.32 & 0.14 & 0.14 & 0.40 & 0.37 & 23.20 & 730.00 & 59.00 & 12.00 & 45.00 & 33.00 & 15.00 & 26.00 \\
\hline 425028 & 42.00 & 5.00 & 28.00 & 401.38 & 0.29 & 0.27 & 0.11 & 0.11 & 0.38 & 0.35 & 27.20 & 650.00 & 44.00 & 19.00 & 39.00 & 29.00 & 14.00 & 19.00 \\
\hline 426047 & 42.00 & 6.00 & 47.00 & 403.07 & 0.36 & 0.32 & 0.09 & 0.09 & 0.39 & 0.37 & 25.40 & 570.00 & 41.00 & 19.00 & 61.00 & 240.00 & 14.00 & 20.00 \\
\hline 427049 & 42.00 & 7.00 & 49.00 & 404.59 & 0.35 & 0.32 & 0.15 & 0.16 & 0.18 & 0.18 & 24.60 & 660.00 & 64.00 & 24.00 & 58.00 & 38.00 & 12.00 & 26.00 \\
\hline 431086 & 43.00 & 1.00 & 86.00 & 405.66 & 0.37 & 0.32 & 0.13 & 0.11 & 0.25 & 0.25 & 22.10 & 710.00 & 53.00 & 32.00 & 50.00 & 180.00 & 13.00 & 24.00 \\
\hline 432029 & 43.00 & 2.00 & 29.00 & 406.59 & 0.42 & 0.35 & 0.09 & 0.09 & 0.10 & 0.10 & 20.60 & 640.00 & 56.00 & 40.00 & 66.00 & 72.00 & 13.00 & 24.00 \\
\hline 433083 & 43.00 & 3.00 & 83.00 & 408.63 & 0.46 & 0.38 & 0.43 & 0.41 & 0.69 & 0.61 & 16.90 & 820.00 & 110.00 & 130.00 & 70.00 & 41.00 & 19.00 & 42.00 \\
\hline 434025 & 43.00 & 4.00 & 25.00 & 409.55 & 0.20 & 0.15 & 14.80 & 16.03 & 2.42 & 2.06 & 16.10 & 620.00 & 740.00 & 25.00 & 34.00 & 27.00 & 21.00 & 350.00 \\
\hline 435018 & 43.00 & 5.00 & 18.00 & 410.98 & 0.36 & 0.32 & 0.19 & 0.18 & 2.01 & 1.93 & 22.60 & 660.00 & 73.00 & 90.00 & 56.00 & 58.00 & - & 29.00 \\
\hline 436027 & 43.00 & 6.00 & 27.00 & 412.57 & 0.51 & 0.43 & 0.14 & 0.14 & 0.11 & 0.11 & 15.00 & 950.00 & 67.00 & 26.00 & 79.00 & 44.00 & 17.00 & 29.00 \\
\hline 437026 & 43.00 & 7.00 & 26.00 & 414.06 & 0.51 & 0.43 & 0.13 & 0.11 & 0.08 & 0.09 & 16.50 & 760.00 & 70.00 & 35.00 & 82.00 & 66.00 & 17.00 & 29.00 \\
\hline 441080 & 44.00 & 1.00 & 80.00 & 415.30 & 0.43 & 0.37 & 0.10 & 0.09 & 0.10 & 0.08 & 18.60 & 740.00 & 63.00 & 14.00 & 71.00 & 69.00 & 15.00 & 26.00 \\
\hline 442082 & 44.00 & 2.00 & 82.00 & 416.82 & $\begin{array}{l}0.43 \\
0.46\end{array}$ & 0.38 & 0.10 & 0.09 & 0.19 & 0.19 & 18.00 & 730.00 & 66.00 & 33.00 & 58,00 & 98.00 & 16.00 & 28.00 \\
\hline
\end{tabular}




\begin{tabular}{|c|c|c|c|c|c|c|c|c|c|c|c|c|c|c|c|c|c|c|}
\hline 443087 & 44.00 & 3.00 & 87.00 & 418.36 & 0.42 & 0.35 & 0.09 & 0.09 & 0.35 & 0.31 & 20.90 & 730.00 & 61.00 & 14.00 & 55.00 & 52.00 & 15.00 & 28.00 \\
\hline 444116 & 44.00 & 4.00 & 116.00 & 420.16 & 0.36 & 0.33 & 0.09 & 0.09 & 0.33 & 0.31 & 23.80 & 680.00 & 49.00 & 14.00 & 59.00 & 37.00 & 14.00 & 23.00 \\
\hline 445074 & 44.00 & 5.00 & 74.00 & 421.24 & 0.31 & 0.27 & 0.10 & 0.09 & 0.31 & 0.30 & 26.90 & 500.00 & 44.00 & 14.00 & 43.00 & 31.00 & 12.00 & 21.00 \\
\hline 446064 & 44.00 & 6.00 & 64.00 & 422.64 & 0.38 & 0.33 & 0.06 & 0.07 & 0.23 & 0.22 & 24.00 & 550.00 & 52.00 & 23.00 & 54.00 & 41.00 & 14.00 & 24.00 \\
\hline 451102 & 45.00 & 1.00 & 102.00 & 425.11 & 0.38 & 0.33 & 0.07 & 0.07 & 0.07 & 0.08 & 20.50 & 850.00 & 58.00 & 23.00 & 62.00 & 57.00 & 15.00 & 24.00 \\
\hline 453055 & 45.00 & 3.00 & 55.00 & 427.65 & 0.35 & 0.30 & 0.07 & 0.07 & 0.08 & 0.09 & 21.10 & 790.00 & 52.00 & 100.00 & 63.00 & 77.00 & 14.00 & 23.00 \\
\hline 461047 & 46.00 & 1.00 & 47.00 & 434.27 & 0.46 & 0.40 & 0.10 & 0.09 & 0.07 & 0.08 & 15.70 & 990.00 & 66.00 & 23.00 & 72.00 & 79.00 & 14.00 & 28.00 \\
\hline 462041 & 46.00 & 2.00 & 41.00 & 435.71 & 0.39 & 0.35 & 0.08 & 0.07 & 0.08 & 0.09 & 18.80 & 1100.00 & 70.00 & 9.00 & 67.00 & 74.00 & 13.00 & 28.00 \\
\hline 463041 & 46.00 & 3.00 & 41.00 & 437.21 & 0.45 & 0.38 & 0.10 & 0.09 & 0.07 & 0.07 & 16.30 & 1100.00 & 68.00 & 310.00 & 74.00 & 81.00 & 13.00 & 28.00 \\
\hline 464041 & 46.00 & 4.00 & 41.00 & 438.71 & 0.57 & 0.50 & 0.08 & 0.07 & 0.05 & 0.06 & 13.30 & 720.00 & 70.00 & 18.00 & 83.00 & 85.00 & 15.00 & 29.00 \\
\hline 465057 & 46.00 & 5.00 & 57.00 & 440.37 & 0.52 & 0.43 & 0.10 & 0.09 & 0.09 & 0.09 & 14.90 & 700.00 & 70.00 & 27.00 & 77.00 & 88.00 & 15.00 & 29.00 \\
\hline 465111 & 46.00 & 5.00 & 111.00 & 440.91 & 0.31 & 0.22 & 11.30 & 12.37 & 0.23 & 0.22 & 14.50 & 730.00 & 860.00 & 15.00 & 48.00 & 69.00 & 11.00 & 410.00 \\
\hline 466057 & 46.00 & 6.00 & 57.00 & 441.87 & 0.50 & 0.43 & 0.09 & 0.09 & 0.08 & 0.08 & 16.20 & 650.00 & 72.00 & 23.00 & 74.00 & 86.00 & 15.00 & 29.00 \\
\hline 471033 & 47.00 & 1.00 & 33.00 & 443.82 & 0.47 & 0.42 & 0.08 & 0.07 & 0.09 & 0.10 & 15.80 & 810.00 & 45.00 & 14.00 & 80.00 & 36.00 & 14.00 & 20.00 \\
\hline 473016 & 47.00 & 3.00 & 16.00 & 446.66 & 0.50 & 0.42 & 0.10 & 0.09 & 0.07 & 0.07 & 14.80 & 840.00 & 73.00 & 45.00 & 76.00 & 74.00 & 15.00 & 30.00 \\
\hline 474021 & 47.00 & 4.00 & 21.00 & 448.21 & 0.46 & 0.40 & 0.07 & 0.07 & 0.30 & 0.28 & 16.70 & 770.00 & 66.00 & 33.00 & 70.00 & 78.00 & 16.00 & 28.00 \\
\hline 475021 & 47.00 & 5.00 & 21.00 & 449.71 & 0.35 & 0.30 & 0.07 & 0.07 & 0.07 & 0.08 & 20.80 & 780.00 & 46.00 & 14.00 & 59.00 & 52.00 & 12.00 & 21.00 \\
\hline 476025 & 47.00 & 6.00 & 25.00 & 451.25 & 0.51 & 0.40 & 0.08 & 0.07 & 0.03 & 0.04 & 8.38 & 820.00 & 130.00 & 34.00 & 96.00 & 130.00 & 15.00 & 55.00 \\
\hline 481007 & 48.00 & 1.00 & 7.00 & 453.27 & - & 0.10 & - & 0.09 & - & 11.48 & - & 260.00 & 36.00 & 32.00 & 21.00 & 18.00 & - & 17.00 \\
\hline 481030 & 48.00 & 1.00 & 30.00 & 453.50 & 0.44 & 0.38 & 0.07 & 0.07 & 0.24 & 0.23 & 13.20 & 1000.00 & 66.00 & 18.00 & 68.00 & 57.00 & 15.00 & 30.00 \\
\hline 482034 & 48.00 & 2.00 & 34.00 & 455.04 & 0.38 & 0.33 & 0.08 & 0.09 & 0.07 & 0.08 & 20.20 & 590.00 & 58.00 & 22.00 & 59.00 & 75.00 & 12.00 & 25.00 \\
\hline 483029 & 48.00 & 3.00 & 29.00 & 456.49 & 0.44 & 0.37 & 0.07 & 0.07 & 0.04 & 0.05 & 13.20 & 890.00 & 68.00 & 59.00 & 71.00 & 39.00 & 14.00 & 29.00 \\
\hline 484032 & 48.00 & 4.00 & 32.00 & 458.02 & 0.46 & 0.40 & 0.13 & 0.11 & 0.12 & 0.12 & 15.40 & 830.00 & 78.00 & 51.00 & 70.00 & 73.00 & 16.00 & 30.00 \\
\hline 485030 & 48.00 & 5.00 & 30.00 & 459.50 & 0.51 & 0.43 & 0.07 & 0.07 & 0.04 & 0.05 & 10.70 & 580.00 & 93.00 & 140.00 & 76.00 & 63.00 & 16.00 & 37.00 \\
\hline 491074 & 49.00 & 1.00 & 74.00 & 463.54 & 0.44 & 0.38 & 0.12 & 0.11 & 0.58 & 0.52 & 16.20 & 870.00 & 57.00 & 32.00 & 70.00 & 64.00 & 18.00 & 24.00 \\
\hline 492066 & 49.00 & 2.00 & 66.00 & 464.96 & 0.44 & 0.38 & 0.08 & 0.09 & 0.09 & 0.09 & 16.10 & 630.00 & 62.00 & 50.00 & 69.00 & 78.00 & 15.00 & 26.00 \\
\hline 493080 & 49.00 & 3.00 & 80.00 & 466.60 & 0.31 & 0.27 & 0.12 & 0.11 & 5.81 & 5.55 & 22.90 & 570.00 & 76.00 & 26.00 & 44.00 & 53.00 & 31.00 & 31.00 \\
\hline 494039 & 49.00 & 4.00 & 39.00 & 467.69 & 0.32 & 0.28 & 0.11 & 0.09 & 2.61 & 2.58 & 21.70 & 780.00 & 69.00 & 100.00 & 47.00 & 55.00 & 27.00 & 28.00 \\
\hline 496043 & 49.00 & 6.00 & 43.00 & 470.73 & 0.27 & 0.25 & 0.07 & 0.07 & 0.17 & 0.17 & 21.10 & 630.00 & 36.00 & 25.00 & 48.00 & 58.00 & 10.00 & 16.00 \\
\hline 501056 & 50.00 & 1.00 & 56.00 & 473.06 & 0.37 & 0.32 & 0.15 & 0.14 & 0.82 & 0.72 & 18.20 & 810.00 & 67.00 & 15.00 & 51.00 & 61.00 & 15.00 & 27.00 \\
\hline 502056 & 50.00 & 2.00 & 56.00 & 474.56 & 0.40 & 0.33 & 0.06 & 0.07 & 0.10 & 0.10 & 18.00 & 610.00 & 52.00 & 45.00 & 62.00 & 84.00 & 12.00 & 23.00 \\
\hline 503036 & 50.00 & 3.00 & 36.00 & 475.86 & 0.41 & 0.35 & 0.07 & 0.07 & 0.09 & 0.09 & 17.00 & 830.00 & 58.00 & 23.00 & 66.00 & 110.00 & 13.00 & 25.00 \\
\hline 504032 & 50.00 & 4.00 & 32.00 & 477.32 & 0.23 & 0.20 & 2.01 & 2.04 & 7.86 & 7.74 & 23.30 & 2100.00 & 160.00 & 31.00 & 41.00 & 50.00 & - & 62.00 \\
\hline 505033 & 50.00 & 5.00 & 33.00 & 478.83 & 0.47 & 0.38 & 0.08 & 0.07 & 0.06 & 0.06 & 8.41 & 640.00 & 87.00 & 36.00 & 90.00 & 68.00 & 16.00 & 37.00 \\
\hline 506012 & 50.00 & 6.00 & 12.00 & 480.12 & 0.24 & 0.22 & 0.15 & 0.14 & 9.58 & 9.42 & 23.30 & 720.00 & 97.00 & 16.00 & 39.00 & 57.00 & - & 38.00 \\
\hline 511031 & 51.00 & 1.00 & 31.00 & 482.40 & 0.40 & 0.35 & 0.07 & 0.07 & 0.23 & 0.22 & 17.00 & 1000.00 & 58.00 & 19.00 & 66.00 & 110.00 & 14.00 & 26.00 \\
\hline 512036 & 51.00 & 2.00 & 36.00 & 483.96 & 0.30 & 0.27 & 0.10 & 0.09 & 0.80 & 0.70 & 22.30 & 840.00 & 53.00 & 23.00 & 52.00 & 65.00 & 16.00 & 23.00 \\
\hline 513035 & 51.00 & 3.00 & 35.00 & 485.45 & 0.32 & 0.28 & 0.06 & 0.07 & 0.21 & 0.21 & 19.60 & 670.00 & 57.00 & 47.00 & 55.00 & 62.00 & 11.00 & 25.00 \\
\hline 514068 & 51.00 & 4.00 & 68.00 & 487.28 & 0.04 & 0.05 & 7.31 & 8.24 & 20.20 & 19.35 & 27.70 & 3200.00 & 620.00 & 16.00 & 17.00 & 8.00 & - & 220.00 \\
\hline 515024 & 51.00 & 5.00 & 24.00 & 488.34 & 0.37 & 0.33 & 0.12 & 0.11 & 2.37 & 2.45 & 18.20 & 940.00 & 53.00 & 21.00 & 60.00 & 50.00 & 18.00 & 23.00 \\
\hline 516083 & 51.00 & 6.00 & 83.00 & 490.43 & - & 0.08 & - & 0.55 & - & 15.48 & - & 490.00 & 120.00 & 39.00 & 20.00 & 14.00 & - & 45.00 \\
\hline 522010 & 52.00 & 2.00 & 10.00 & 493.40 & 0.24 & 0.22 & 0.78 & 0.80 & 5.48 & 5.29 & 24.00 & 610.00 & 110.00 & 14.00 & 44.00 & 160.00 & 14.00 & 44.00 \\
\hline 523098 & 52.00 & 3.00 & 98.00 & 495.78 & 0.22 & 0.20 & 0.11 & 0.11 & 3.28 & 3.22 & 22.30 & 780.00 & 45.00 & 11.00 & 44.00 & 59.00 & - & 19.00 \\
\hline 524056 & 52.00 & 4.00 & 56.00 & 496.86 & 0.30 & 0.27 & 0.08 & 0.09 & 0.13 & 0.13 & 22.40 & 700.00 & 59.00 & 70.00 & 55.00 & 71.00 & 12.00 & 24.00 \\
\hline 525069 & 52.00 & 5.00 & 69.00 & 498.49 & 0.34 & 0.30 & 0.10 & 0.09 & 1.67 & 1.68 & 21.70 & 730.00 & 55.00 & 23.00 & 41.00 & 69.00 & - & 24.00 \\
\hline 526076 & 52.00 & 6.00 & 76.00 & 500.06 & 0.48 & 0.42 & 0.06 & 0.07 & 0.03 & 0.04 & 8.10 & 720.00 & 110.00 & 18.00 & 97.00 & 71.00 & 17.00 & 44.00 \\
\hline 531047 & 53.00 & 1.00 & 47.00 & 501.87 & 0.42 & 0.37 & 0.11 & 0.09 & 0.15 & 0.15 & 17.30 & 620.00 & 78.00 & 17.00 & 54.00 & 97.00 & 15.00 & 32.00 \\
\hline 532008 & 53.00 & 2.00 & 8.00 & 502.98 & 0.50 & 0.43 & 0.06 & 0.07 & $0.02 \mathrm{~L}$ & 0.03 & 8.81 & 650.00 & 86.00 & 86.00 & 94.00 & 50.00 & 17.00 & 38.00 \\
\hline 533055 & 53.00 & 3.00 & 55.00 & 504.95 & 0.38 & 0.32 & 0.07 & 0.07 & 0.13 & 0.13 & 16.60 & 580.00 & 48.00 & 190.00 & 60.00 & 70.00 & 13.00 & 22.00 \\
\hline 534079 & 53.00 & 4.00 & 79.00 & 506.45 & - & 0.05 & - & 7.56 & - & 16.77 & - & 1700.00 & 600.00 & 61.00 & 18.00 & 21.00 & - & 180.00 \\
\hline 535018 & 53.00 & 5.00 & 18.00 & 507.58 & 0.39 & 0.33 & 0.09 & 0.09 & 0.18 & 0.17 & 18.60 & 590.00 & 59.00 & 62.00 & 52.00 & 75.00 & 12.00 & 26.00 \\
\hline 541025 & 54.00 & 1.00 & 25.00 & 511.35 & 0.39 & 0.33 & 0.19 & 0.18 & 3.24 & 3.22 & 19.70 & 690.00 & 70.00 & 19.00 & 57.00 & 51.00 & 19.00 & 28.00 \\
\hline 542013 & 54.00 & 2.00 & 13.00 & 512.73 & 0.35 & 0.32 & 0.09 & 0.09 & 1.36 & 1.12 & 20.10 & 680.00 & 44.00 & 27.00 & 59.00 & 80.00 & 19.00 & 19.00 \\
\hline 543016 & 54.00 & 3.00 & 16.00 & 514.26 & 0.54 & 0.47 & 0.09 & 0.09 & 0.08 & 0.08 & 14.00 & 670.00 & 77.00 & 35.00 & 80.00 & 78.00 & 17.00 & 32.00 \\
\hline 544123 & 54.00 & 4.00 & 123.00 & 516.83 & 0.45 & 0.40 & 0.06 & 0.05 & 0.03 & 0.04 & 7.80 & 760.00 & 110.00 & 21.00 & 83.00 & 61.00 & 18.00 & 43.00 \\
\hline 545011 & 54.00 & 5.00 & 11.00 & 517.20 & 0.48 & 0.42 & 0.11 & 0.09 & 0.54 & 0.49 & 14.60 & 640.00 & 70.00 & 21.00 & 73.00 & 64.00 & 17.00 & 29.00 \\
\hline 546014 & 54.00 & 6.00 & 14.00 & 518.74 & 0.30 & 0.28 & 0.11 & 0.09 & 0.20 & 0.19 & 21.10 & 610.00 & 50.00 & 19.00 & 48.00 & 46.00 & 11.00 & 21.00 \\
\hline
\end{tabular}




\begin{tabular}{|c|c|c|c|c|c|c|c|c|c|c|c|c|c|c|c|c|c|c|}
\hline Sample & Core & Section & Interval & $\begin{array}{l}\text { Depth } \\
\text { (m) }\end{array}$ & $\underset{(\mathrm{ppm})}{\mathrm{Li}}$ & $\underset{(\mathrm{ppm})}{\mathrm{Nb}}$ & $\begin{array}{c}\mathrm{Nd} \\
(\mathrm{ppm})\end{array}$ & $\begin{array}{c}\mathrm{Ni} \\
(\mathrm{ppm})\end{array}$ & $\begin{array}{c}\mathrm{Pb} \\
(\mathrm{ppm})\end{array}$ & $\underset{(\mathrm{ppm})}{\mathrm{Sc}}$ & $\underset{(\mathrm{ppm})}{\mathrm{Sr}}$ & $\begin{array}{c}\text { Th } \\
(\mathrm{ppm})\end{array}$ & $\underset{(\mathrm{ppm})}{\mathrm{V}}$ & $\underset{(\mathrm{ppm})}{\mathrm{Y}}$ & $\begin{array}{c}\mathrm{Yb} \\
(\mathrm{ppm})\end{array}$ & $\begin{array}{c}\mathrm{Zn} \\
(\mathrm{ppm})\end{array}$ & $\begin{array}{c}\mathrm{CaCO}_{3} \text {-ca } \\
(\%)\end{array}$ & $\begin{array}{c}\mathrm{CaCO}_{3}-\mathrm{cc} \\
(\%)\end{array}$ \\
\hline 281089 & 28.00 & 1.00 & 89.00 & 260.99 & 30.00 & 6.00 & 23.00 & 51.00 & 14.00 & 10.00 & 780.00 & 9.00 & 150.00 & 14.00 & 1.00 & 86.00 & 33.21 & 30.83 \\
\hline 282052 & 28.00 & 2.00 & 52.00 & 262.12 & 25.00 & 4.00 & 16.00 & 110.00 & 19.00 & 9.00 & 840.00 & 7.00 & 110.00 & 11.00 & 1.00 & 58.00 & 37.86 & 35.92 \\
\hline 283054 & 28.00 & 3.00 & 54.00 & 263.65 & 24.00 & 5.00 & 16.00 & 43.00 & 11.00 & 8.00 & 1100.00 & 5.00 & 110.00 & 12.00 & 1.00 & 74.00 & 51.61 & 48.25 \\
\hline 284068 & 28.00 & 4.00 & 68.00 & 265.28 & 34.00 & 5.00 & 23.00 & 84.00 & 16.00 & 12.00 & 520.00 & 9.00 & 130.00 & 14.00 & 2.00 & 140.00 & 21.25 & 20.00 \\
\hline 301051 & 30.00 & 1.00 & 51.00 & 279.93 & 23.00 & 4.00 & 17.00 & 42.00 & 10.00 & 9.00 & 800.00 & 15.00 & 86.00 & 15.00 & 2.00 & 89.00 & 41.79 & 50.92 \\
\hline 302092 & 30.00 & 2.00 & 92.00 & 281.82 & 28.00 & 4.00 & 19.00 & 58.00 & 14.00 & 9.00 & 940.00 & 7.00 & 110.00 & 12.00 & 1.00 & 140.00 & 43.93 & 40.92 \\
\hline 303045 & 30.00 & 3.00 & 45.00 & 282.85 & 25.00 & $<4.00$ & 16.00 & 39.00 & 13.00 & 8.00 & 1000.00 & 6.00 & 99.00 & 15.00 & 1.00 & 67.00 & 52.14 & 49.67 \\
\hline 304098 & 30.00 & 4.00 & 98.00 & 284.88 & 28.00 & $<4.00$ & 22.00 & 41.00 & 15.00 & 10.00 & 840.00 & 7.00 & 120.00 & 20.00 & 2.00 & 82.00 & 41.25 & 38.67 \\
\hline 305112 & 30.00 & 5.00 & 112.00 & 286.52 & 30.00 & 5.00 & 20.00 & 53.00 & 17.00 & 10.00 & 780.00 & 7.00 & 110.00 & 17.00 & 2.00 & 92.00 & 37.14 & 34.42 \\
\hline 307029 & 30.00 & 7.00 & 29.00 & 288.69 & 34.00 & 6.00 & 22.00 & 77.00 & 16.00 & 12.00 & 620.00 & 9.00 & 140.00 & 12.00 & 1.00 & 92.00 & 29.46 & 27.17 \\
\hline 311029 & 31.00 & 1.00 & 29.00 & 289.29 & 27.00 & 5.00 & 20.00 & 64.00 & 14.00 & 11.00 & 780.00 & 8.00 & 120.00 & 15.00 & 2.00 & 85.00 & 36.25 & 33.58 \\
\hline 312102 & 31.00 & 2.00 & 102.00 & 291.51 & 26.00 & 6.00 & 19.00 & 55.00 & 12.00 & 10.00 & 800.00 & 7.00 & 110.00 & 14.00 & 2.00 & 93.00 & 35.71 & 37.17 \\
\hline 321049 & 32.00 & 1.00 & 49.00 & 299.09 & 21.00 & 5.00 & 15.00 & 36.00 & 11.00 & 8.00 & 1000.00 & 6.00 & 110.00 & 12.00 & 1.00 & 63.00 & 45.00 & 42.42 \\
\hline 322032 & 32.00 & 2.00 & 32.00 & 300.42 & 20.00 & $<4.00$ & 16.00 & 61.00 & 14.00 & 8.00 & 860.00 & 5.00 & 100.00 & 12.00 & 1.00 & 61.00 & 37.32 & 34.08 \\
\hline 332018 & 33.00 & 2.00 & 18.00 & 309.98 & 23.00 & 5.00 & 17.00 & 39.00 & 12.00 & 9.00 & 970.00 & 44.00 & 120.00 & 10.00 & 1.00 & 67.00 & 41.79 & 36.67 \\
\hline 351028 & 35.00 & 1.00 & 28.00 & 327.88 & 32.00 & $<4.00$ & 22.00 & 53.00 & 12.00 & 11.00 & 550.00 & 34.00 & 130.00 & 13.00 & 1.00 & 87.00 & 28.93 & 26.58 \\
\hline 352029 & 35.00 & 2.00 & 29.00 & 329.39 & 31.00 & 7.00 & 22.00 & 52.00 & 14.00 & 11.00 & 590.00 & 7.00 & 140.00 & 15.00 & 2.00 & 100.00 & 30.54 & 28.33 \\
\hline 353024 & 35.00 & 3.00 & 24.00 & 332.63 & 32.00 & $<4.00$ & 22.00 & 55.00 & 16.00 & 11.00 & 610.00 & 9.00 & 140.00 & 13.00 & 2.00 & 110.00 & 30.36 & 28.17 \\
\hline 361068 & 36.00 & 1.00 & 68.00 & 337.88 & 31.00 & $<4.00$ & 26.00 & 78.00 & 16.00 & 11.00 & 590.00 & 6.00 & 120.00 & 18.00 & 2.00 & 91.00 & 30.54 & 26.33 \\
\hline 362068 & 36.00 & 2.00 & 68.00 & 339.38 & 32.00 & 6.00 & 23.00 & 84.00 & 13.00 & 12.00 & 590.00 & 8.00 & 130.00 & 13.00 & 2.00 & 97.00 & 27.50 & 25.58 \\
\hline 364068 & 36.00 & 4.00 & 68.00 & 342.38 & 29.00 & 5.00 & 25.00 & 72.00 & 19.00 & 11.00 & 690.00 & 9.00 & 130.00 & 16.00 & 2.00 & 100.00 & 28.75 & 26.92 \\
\hline 372014 & 37.00 & 2.00 & 15.00 & 348.35 & 31.00 & 6.00 & 20.00 & 89.00 & 19.00 & 10.00 & 740.00 & 8.00 & 140.00 & 12.00 & 1.00 & 110.00 & 31.96 & 23.17 \\
\hline 373015 & 37.00 & 3.00 & 15.00 & 349.85 & 32.00 & 6.00 & 20.00 & 45.00 & 17.00 & 10.00 & 810.00 & 8.00 & 130.00 & 12.00 & 1.00 & 83.00 & 35.18 & 32.00 \\
\hline 374015 & 37.00 & 4.00 & 15.00 & 351.35 & 29.00 & $<4.00$ & 16.00 & 37.00 & 11.00 & 9.00 & 810.00 & 6.00 & 120.00 & 10.00 & 1.00 & 87.00 & 37.32 & 35.17 \\
\hline 381036 & 38.00 & 1.00 & 36.00 & 356.76 & 26.00 & $<4.00$ & 17.00 & 46.00 & 12.00 & 9.00 & 860.00 & 6.00 & 100.00 & 11.00 & 1.00 & 69.00 & 41.25 & 38.33 \\
\hline 382040 & 38.00 & 2.00 & 39.00 & 358.30 & 29.00 & 4.00 & 20.00 & 39.00 & 13.00 & 10.00 & 710.00 & 7.00 & 120.00 & 11.00 & 1.00 & 83.00 & 32.32 & 30.25 \\
\hline 383041 & 38.00 & 3.00 & 41.00 & 359.81 & 29.00 & 5.00 & 16.00 & 49.00 & 14.00 & 10.00 & 710.00 & 9.00 & 130.00 & 11.00 & 1.00 & 72.00 & 31.96 & 29.58 \\
\hline 384041 & 38.00 & 4.00 & 41.00 & 361.31 & 27.00 & 5.00 & 13.00 & 52.00 & 12.00 & 9.00 & 850.00 & 7.00 & 110.00 & 10.00 & 1.00 & 68.00 & 38.75 & 37.33 \\
\hline 385041 & 38.00 & 5.00 & 41.00 & 362.81 & 32.00 & 6.00 & 22.00 & 50.00 & 15.00 & 11.00 & 610.00 & 9.00 & 140.00 & 12.00 & 2.00 & 91.00 & 26.61 & 24.42 \\
\hline 386066 & 38.00 & 6.00 & 67.00 & 364.56 & 11.00 & $<4.00$ & 13.00 & 27.00 & 4.00 & 5.00 & 200.00 & $<4.00$ & 25.00 & 27.00 & 2.00 & 29.00 & 17.00 & 70.75 \\
\hline 391017 & 39.00 & 1.00 & 17.00 & 366.27 & 38.00 & 8.00 & 34.00 & 120.00 & 27.00 & 12.00 & 450.00 & 8.00 & 160.00 & 21.00 & 2.00 & 100.00 & 17.09 & 15.50 \\
\hline 392017 & 39.00 & 2.00 & 17.00 & 367.77 & 32.00 & 6.00 & 23.00 & 74.00 & 18.00 & 11.00 & 670.00 & 7.00 & 130.00 & 14.00 & 2.00 & 77.00 & 28.04 & 26.08 \\
\hline 393019 & 39.00 & 3.00 & 19.00 & 369.29 & 32.00 & 6.00 & 22.00 & 56.00 & 16.00 & 11.00 & 690.00 & 8.00 & 130.00 & 13.00 & 2.00 & 82.00 & 28.75 & 26.75 \\
\hline 411030 & 41.00 & 1.00 & 30.00 & 385.70 & 26.00 & 4.00 & 24.00 & 45.00 & 17.00 & 10.00 & 600.00 & 5.00 & 110.00 & 17.00 & 2.00 & 64.00 & 29.29 & 40.17 \\
\hline 412030 & 41.00 & 2.00 & 30.00 & 387.20 & 25.00 & $<4.00$ & 16.00 & 41.00 & 13.00 & 10.00 & 880.00 & 14.00 & 110.00 & 10.00 & 1.00 & 75.00 & 42.14 & 38.83 \\
\hline 413030 & 41.00 & 3.00 & 30.00 & 388.70 & 25.00 & $<4.00$ & 20.00 & 53.00 & 18.00 & 9.00 & 910.00 & 6.00 & 110.00 & 15.00 & 2.00 & 83.00 & 42.86 & 40.17 \\
\hline 414030 & 41.00 & 4.00 & 30.00 & 390.20 & 28.00 & 5.00 & 20.00 & 54.00 & 14.00 & 11.00 & 860.00 & 9.00 & 140.00 & 11.00 & 1.00 & 76.00 & 35.71 & 33.08 \\
\hline 415030 & 41.00 & 5.00 & 30.00 & 391.70 & 21.00 & $<4.00$ & 20.00 & 110.00 & 14.00 & 9.00 & 640.00 & 4.00 & 88.00 & 18.00 & 2.00 & 77.00 & 30.89 & 47.92 \\
\hline 416070 & 41.00 & 6.00 & 70.00 & 393.60 & 21.00 & $<4.00$ & 22.00 & 44.00 & 9.00 & 9.00 & 600.00 & 5.00 & 87.00 & 21.00 & 2.00 & 82.00 & 30.71 & 54.58 \\
\hline 421042 & 42.00 & 1.00 & 42.00 & 395.52 & 29.00 & 5.00 & 17.00 & 46.00 & 18.00 & 11.00 & 850.00 & 8.00 & 140.00 & 11.00 & 1.00 & 85.00 & 39.29 & 36.83 \\
\hline 422048 & 42.00 & 2.00 & 48.00 & 397.07 & 26.00 & 5.00 & 22.00 & 44.00 & 15.00 & 10.00 & 950.00 & 8.00 & 120.00 & 15.00 & 2.00 & 83.00 & 41.79 & 40.25 \\
\hline 424014 & 42.00 & 4.00 & 14.00 & 399.74 & 26.00 & 5.00 & 22.00 & 42.00 & 16.00 & 10.00 & 980.00 & 8.00 & 110.00 & 16.00 & 2.00 & 72.00 & 43.21 & 41.92 \\
\hline 425028 & 42.00 & 5.00 & 28.00 & 401.38 & 23.00 & 4.00 & 17.00 & 37.00 & 13.00 & 8.00 & 1200.00 & 8.00 & 100.00 & 12.00 & 1.00 & 59.00 & 52.32 & 50.50 \\
\hline 433083 & 43.00 & 3.00 & 83.00 & 408.63 & 30.00 & 5.00 & 41.00 & 88.00 & 18.00 & 12.00 & 580.00 & 9.00 & 130.00 & 36.00 & 3.00 & 83.00 & 25.89 & 24.00 \\
\hline 434025 & 43.00 & 4.00 & 25.00 & 409.55 & 16.00 & 5.00 & 260.00 & 36.00 & 9.00 & 14.00 & 1100.00 & $<4.00$ & 71.00 & 480.00 & 28.00 & 48.00 & 55.89 & 24.17 \\
\hline 435018 & 43.00 & 5.00 & 18.00 & 410.98 & 26.00 & 5.00 & 27.00 & 60.00 & 12.00 & 11.00 & 820.00 & 6.00 & 98.00 & 22.00 & 2.00 & 95.00 & 39.46 & 41.50 \\
\hline 436027 & 43.00 & 6.00 & 27.00 & 412.57 & 31.00 & 7.00 & 28.00 & 84.00 & 20.00 & 13.00 & 540.00 & 9.00 & 140.00 & 18.00 & 2.00 & 70.00 & 21.61 & 20.00 \\
\hline 437026 & 43.00 & 7.00 & 26.00 & 414.06 & 33.00 & 7.00 & 29.00 & 65.00 & 22.00 & 13.00 & 600.00 & 9.00 & 190.00 & 18.00 & 2.00 & 110.00 & 23.75 & 21.42 \\
\hline 441080 & 44.00 & 1.00 & 80.00 & 415.30 & 30.00 & 6.00 & 23.00 & 55.00 & 15.00 & 12.00 & 760.00 & 8.00 & 140.00 & 15.00 & 2.00 & 90.00 & 33.39 & 31.25 \\
\hline 442082 & 44.00 & 2.00 & 82.00 & 416.82 & 31.00 & 9.00 & 26.00 & 80.00 & 20.00 & 12.00 & 680.00 & 8.00 & 130.00 & 16.00 & 2.00 & 130.00 & 31.25 & 29.67 \\
\hline
\end{tabular}




\begin{tabular}{|c|c|c|c|c|c|c|c|c|c|c|c|c|c|c|c|c|c|c|}
\hline 443087 & 44.00 & 3.00 & 87.00 & 418.36 & 29.00 & 5.00 & 22.00 & 43.00 & 18.00 & 11.00 & 740.00 & 8.00 & 99.00 & 14.00 & 2.00 & 73.00 & 38.93 & 36.50 \\
\hline 444116 & 44.00 & 4.00 & 116.00 & 420.16 & 28.00 & 7.00 & 18.00 & 43.00 & 11.00 & 10.00 & 890.00 & 8.00 & 89.00 & 13.00 & 1.00 & 52.00 & 45.71 & 43.92 \\
\hline 445074 & 44.00 & 5.00 & 74.00 & 421.24 & 24.00 & $<4.00$ & 17.00 & 31.00 & 10.00 & 8.00 & 950.00 & 7.00 & 83.00 & 13.00 & 1.00 & 52.00 & 53.39 & 50.17 \\
\hline 446064 & 44.00 & 6.00 & 64.00 & 422.64 & 25.00 & 5.00 & 17.00 & 43.00 & 12.00 & 9.00 & 890.00 & 7.00 & 85.00 & 10.00 & 1.00 & 54.00 & 45.36 & 43.00 \\
\hline 451102 & 45.00 & 1.00 & 102.00 & 425.11 & 28.00 & 5.00 & 21.00 & 54.00 & 13.00 & 11.00 & 760.00 & 8.00 & 110.00 & 14.00 & 2.00 & 91.00 & 36.61 & 34.75 \\
\hline 453055 & 45.00 & 3.00 & 55.00 & 427.65 & 27.00 & 4.00 & 19.00 & 54.00 & 14.00 & 11.00 & 800.00 & 7.00 & 110.00 & 13.00 & 1.00 & 88.00 & 39.82 & 34.08 \\
\hline 461047 & 46.00 & 1.00 & 47.00 & 434.27 & 31.00 & 6.00 & 27.00 & 68.00 & 17.00 & 12.00 & 530.00 & 9.00 & 150.00 & 19.00 & 2.00 & 75.00 & 25.00 & 22.75 \\
\hline 462041 & 46.00 & 2.00 & 41.00 & 435.71 & 29.00 & 5.00 & 25.00 & 43.00 & 18.00 & 11.00 & 760.00 & 8.00 & 170.00 & 16.00 & 2.00 & 44.00 & 34.11 & 32.08 \\
\hline 463041 & 46.00 & 3.00 & 41.00 & 437.21 & 30.00 & $<4.00$ & 29.00 & 77.00 & 19.00 & 12.00 & 510.00 & 11.00 & 140.00 & 18.00 & 2.00 & 65.00 & 24.64 & 21.67 \\
\hline 464041 & 46.00 & 4.00 & 41.00 & 438.71 & 35.00 & 7.00 & 29.00 & 49.00 & 19.00 & 14.00 & 390.00 & 9.00 & 220.00 & 16.00 & 2.00 & 100.00 & 17.75 & 16.17 \\
\hline 465057 & 46.00 & 5.00 & 57.00 & 440.37 & 31.00 & 7.00 & 27.00 & 78.00 & 22.00 & 12.00 & 490.00 & 9.00 & 160.00 & 14.00 & 2.00 & 80.00 & 23.75 & 21.92 \\
\hline 465111 & 46.00 & 5.00 & 111.00 & 440.91 & 20.00 & 5.00 & 280.00 & 47.00 & 14.00 & 14.00 & 910.00 & $<4.00$ & 110.00 & 430.00 & 24.00 & 62.00 & 50.18 & 21.92 \\
\hline 466057 & 46.00 & 6.00 & 57.00 & 441.87 & 31.00 & 9.00 & 27.00 & 71.00 & 16.00 & 12.00 & 520.00 & 10.00 & 160.00 & 16.00 & 2.00 & 100.00 & 25.36 & 23.33 \\
\hline 471033 & 47.00 & 1.00 & 33.00 & 443.82 & 32.00 & 7.00 & 18.00 & 55.00 & 18.00 & 12.00 & 490.00 & 9.00 & 160.00 & 11.00 & 1.00 & 50.00 & 23.39 & 22.00 \\
\hline 473016 & 47.00 & 3.00 & 16.00 & 446.66 & 33.00 & 6.00 & 28.00 & 46.00 & 16.00 & 13.00 & 450.00 & 10.00 & 160.00 & 18.00 & 2.00 & 88.00 & 21.79 & 19.83 \\
\hline 474021 & 47.00 & 4.00 & 21.00 & 448.21 & 32.00 & 7.00 & 26.00 & 53.00 & 12.00 & 14.00 & 550.00 & 11.00 & 160.00 & 17.00 & 2.00 & 110.00 & 24.46 & 25.00 \\
\hline 475021 & 47.00 & 5.00 & 21.00 & 449.71 & 27.00 & 6.00 & 18.00 & 40.00 & 14.00 & 10.00 & 780.00 & 9.00 & 120.00 & 15.00 & 2.00 & 56.00 & 37.86 & 35.75 \\
\hline 476025 & 47.00 & 6.00 & 25.00 & 451.25 & 37.00 & 8.00 & 54.00 & 86.00 & 27.00 & 12.00 & 150.00 & 17.00 & 290.00 & 27.00 & 3.00 & 100.00 & 1.73 & 0.75 \\
\hline 481007 & 48.00 & 1.00 & 7.00 & 453.27 & 14.00 & $<4.00$ & 13.00 & 26.00 & 6.00 & 4.00 & 120.00 & $<4.00$ & 41.00 & 17.00 & 2.00 & 69.00 & 11.00 & 69.75 \\
\hline 481030 & 48.00 & 1.00 & 30.00 & 453.50 & 41.00 & 6.00 & 26.00 & 59.00 & 18.00 & 13.00 & 410.00 & 11.00 & 170.00 & 13.00 & 2.00 & 73.00 & 16.50 & 15.67 \\
\hline 482034 & 48.00 & 2.00 & 34.00 & 455.04 & 31.00 & $<4.00$ & 24.00 & 48.00 & 11.00 & 11.00 & 730.00 & 6.00 & 140.00 & 17.00 & 2.00 & 110.00 & 36.43 & 34.00 \\
\hline 483029 & 48.00 & 3.00 & 29.00 & 456.49 & 36.00 & 6.00 & 26.00 & 90.00 & 19.00 & 13.00 & 440.00 & 8.00 & 130.00 & 15.00 & 2.00 & 69.00 & 18.93 & 16.75 \\
\hline 484032 & 48.00 & 4.00 & 32.00 & 458.02 & 35.00 & 6.00 & 28.00 & 78.00 & 20.00 & 13.00 & 530.00 & 9.00 & 130.00 & 19.00 & 2.00 & 99.00 & 24.46 & 22.08 \\
\hline 485030 & 48.00 & 5.00 & 30.00 & 459.50 & 37.00 & 6.00 & 34.00 & 81.00 & 20.00 & 14.00 & 310.00 & 11.00 & 140.00 & 15.00 & 2.00 & 110.00 & 11.32 & 9.83 \\
\hline 491074 & 49.00 & 1.00 & 74.00 & 463.54 & 32.00 & 6.00 & 24.00 & 54.00 & 17.00 & 13.00 & 560.00 & 11.00 & 140.00 & 17.00 & 2.00 & 92.00 & 25.36 & 23.75 \\
\hline 492066 & 49.00 & 2.00 & 66.00 & 464.96 & 31.00 & 6.00 & 24.00 & 67.00 & 17.00 & 12.00 & 590.00 & 9.00 & 160.00 & 15.00 & 2.00 & 100.00 & 24.64 & 22.57 \\
\hline 493080 & 49.00 & 3.00 & 80.00 & 466.60 & 24.00 & 5.00 & 27.00 & 38.00 & 10.00 & 10.00 & 600.00 & 18.00 & 100.00 & 21.00 & 3.00 & 62.00 & 30.89 & 41.83 \\
\hline 494039 & 49.00 & 4.00 & 39.00 & 467.69 & 25.00 & 5.00 & 26.00 & 79.00 & 21.00 & 10.00 & 670.00 & 13.00 & 120.00 & 18.00 & 2.00 & 61.00 & 33.57 & 35.00 \\
\hline 496043 & 49.00 & 6.00 & 43.00 & 470.73 & 28.00 & $<4.00$ & 12.00 & 49.00 & 11.00 & 9.00 & 790.00 & 6.00 & 110.00 & 10.00 & 1.00 & 83.00 & 43.36 & 38.33 \\
\hline 501056 & 50.00 & 1.00 & 56.00 & 473.06 & 28.00 & 5.00 & 30.00 & 43.00 & 14.00 & 11.00 & 620.00 & 7.00 & 120.00 & 20.00 & 2.00 & 81.00 & 30.54 & 29.67 \\
\hline 502056 & 50.00 & 2.00 & 56.00 & 474.56 & 29.00 & 5.00 & 21.00 & 71.00 & 20.00 & 12.00 & 580.00 & 9.00 & 140.00 & 12.00 & 2.00 & 92.00 & 27.86 & 25.25 \\
\hline 503036 & 50.00 & 3.00 & 36.00 & 475.86 & 30.00 & 4.00 & 23.00 & 56.00 & 18.00 & 12.00 & 580.00 & 9.00 & 170.00 & 12.00 & 1.00 & 120.00 & 27.86 & 24.75 \\
\hline 504032 & 50.00 & 4.00 & 32.00 & 477.32 & 21.00 & 4.00 & 67.00 & 47.00 & 10.00 & 9.00 & 640.00 & $<4.00$ & 95.00 & 82.00 & 5.00 & 73.00 & 31.43 & 43.83 \\
\hline 505033 & 50.00 & 5.00 & 33.00 & 478.83 & 38.00 & 6.00 & 32.00 & 59.00 & 20.00 & 12.00 & 190.00 & 14.00 & 190.00 & 11.00 & 1.00 & 80.00 & 4.55 & 3.42 \\
\hline 506012 & 50.00 & 6.00 & 12.00 & 480.12 & 22.00 & $<4.00$ & 34.00 & 48.00 & 12.00 & 9.00 & 500.00 & 4.00 & 100.00 & 30.00 & 4.00 & 85.00 & 26.07 & 43.92 \\
\hline 511031 & 51.00 & 1.00 & 31.00 & 482.40 & 29.00 & 5.00 & 23.00 & 58.00 & 16.00 & 11.00 & 630.00 & 10.00 & 160.00 & 13.00 & 2.00 & 120.00 & 28.21 & 25.33 \\
\hline 512036 & 51.00 & 2.00 & 36.00 & 483.96 & 24.00 & 4.00 & 24.00 & 52.00 & 14.00 & 10.00 & 820.00 & 8.00 & 120.00 & 16.00 & 2.00 & 87.00 & 38.04 & 36.08 \\
\hline 513035 & 51.00 & 3.00 & 35.00 & 485.45 & 25.00 & 6.00 & 23.00 & 61.00 & 13.00 & 10.00 & 780.00 & 8.00 & 110.00 & 13.00 & 2.00 & 76.00 & 38.39 & 34.58 \\
\hline 514068 & 51.00 & 4.00 & 68.00 & 487.28 & 9.00 & $<4.00$ & 300.00 & 15.00 & $<4.00$ & 7.00 & 760.00 & 43.00 & 28.00 & 300.00 & 16.00 & 85.00 & 33.75 & 63.92 \\
\hline 515024 & 51.00 & 5.00 & 24.00 & 488.34 & 27.00 & 5.00 & 24.00 & 59.00 & 16.00 & 11.00 & 520.00 & 8.00 & 120.00 & 20.00 & 2.00 & 54.00 & 25.36 & 28.00 \\
\hline 516083 & 51.00 & 6.00 & 83.00 & 490.43 & 15.00 & $<4.00$ & 57.00 & 39.00 & 8.00 & 7.00 & 350.00 & $<4.00$ & 59.00 & 54.00 & 5.00 & 38.00 & 22.50 & 65.67 \\
\hline 522010 & 52.00 & 2.00 & 10.00 & 493.40 & 23.00 & $<4.00$ & 48.00 & 29.00 & 19.00 & 9.00 & 730.00 & $<4.00$ & 150.00 & 49.00 & 3.00 & 300.00 & 35.18 & 45.83 \\
\hline 523098 & 52.00 & 3.00 & 98.00 & 495.78 & 29.00 & $<4.00$ & 17.00 & 23.00 & 8.00 & 7.00 & 730.00 & 5.00 & 93.00 & 18.00 & 2.00 & 33.00 & 35.36 & 41.17 \\
\hline 524056 & 52.00 & 4.00 & 56.00 & 496.86 & 26.00 & $<4.00$ & 21.00 & 35.00 & 10.00 & 9.00 & 910.00 & 7.00 & 110.00 & 16.00 & 2.00 & 51.00 & 41.96 & 39.08 \\
\hline 525069 & 52.00 & 5.00 & 69.00 & 498.49 & 24.00 & 4.00 & 22.00 & 51.00 & 14.00 & 10.00 & 790.00 & 8.00 & 130.00 & 20.00 & 2.00 & 75.00 & 37.86 & 39.25 \\
\hline 526076 & 52.00 & 6.00 & 76.00 & 500.06 & 34.00 & 8.00 & 41.00 & 58.00 & 18.00 & 13.00 & 170.00 & 17.00 & 260.00 & 14.00 & 2.00 & 83.00 & 2.39 & 1.50 \\
\hline 531047 & 53.00 & 1.00 & 47.00 & 501.87 & 28.00 & 5.00 & 30.00 & 60.00 & 28.00 & 12.00 & 670.00 & 10.00 & 160.00 & 18.00 & 2.00 & 110.00 & 28.93 & 26.08 \\
\hline 532008 & 53.00 & 2.00 & 8.00 & 502.98 & 36.00 & 10.00 & 33.00 & 51.00 & 10.00 & 13.00 & 150.00 & 17.00 & 230.00 & 12.00 & 2.00 & 65.00 & 1.38 & 0.42 \\
\hline 533055 & 53.00 & 3.00 & 55.00 & 504.95 & 30.00 & 5.00 & 19.00 & 260.00 & 24.00 & 11.00 & 580.00 & 8.00 & 150.00 & 12.00 & 1.00 & 68.00 & 26.61 & 24.25 \\
\hline 534079 & 53.00 & 4.00 & 79.00 & 506.45 & 10.00 & $<4.00$ & 340.00 & 18.00 & $<4.00$ & 8.00 & 590.00 & $<4.00$ & 60.00 & 330.00 & 20.00 & 380.00 & 27.50 & 58.92 \\
\hline 535018 & 53.00 & 5.00 & 18.00 & 507.58 & 27.00 & $<4.00$ & 26.00 & 62.00 & 14.00 & 11.00 & 680.00 & 7.00 & 130.00 & 16.00 & 2.00 & 82.00 & 33.04 & 30.42 \\
\hline 541025 & 54.00 & 1.00 & 25.00 & 511.35 & 24.00 & 4.00 & 29.00 & 54.00 & 16.00 & 11.00 & 570.00 & 7.00 & 130.00 & 20.00 & 2.00 & 67.00 & 27.50 & 35.58 \\
\hline 542013 & 54.00 & 2.00 & 13.00 & 512.73 & 27.00 & 5.00 & 15.00 & 70.00 & 14.00 & 10.00 & 730.00 & 7.00 & 120.00 & 13.00 & 2.00 & 68.00 & 33.93 & 30.75 \\
\hline 543016 & 54.00 & 3.00 & 16.00 & 514.26 & 30.00 & 7.00 & 28.00 & 73.00 & 15.00 & 14.00 & 490.00 & 12.00 & 170.00 & 16.00 & 2.00 & 89.00 & 20.54 & 18.33 \\
\hline 544123 & 54.00 & 4.00 & 123.00 & 516.83 & 38.00 & 7.00 & 38.00 & 75.00 & 20.00 & 13.00 & 190.00 & 19.00 & 190.00 & 12.00 & 2.00 & 70.00 & 4.64 & 3.67 \\
\hline 545011 & 54.00 & 5.00 & 11.00 & 517.20 & 31.00 & 6.00 & 27.00 & 64.00 & 17.00 & 12.00 & 550.00 & 9.00 & 150.00 & 17.00 & 2.00 & 90.00 & 22.50 & 20.75 \\
\hline 546014 & 54.00 & 6.00 & 14.00 & 518.74 & 25.00 & 4.00 & 19.00 & 41.00 & 14.00 & 9.00 & 840.00 & 7.00 & 110.00 & 14.00 & 1.00 & 68.00 & 40.00 & 37.92 \\
\hline
\end{tabular}



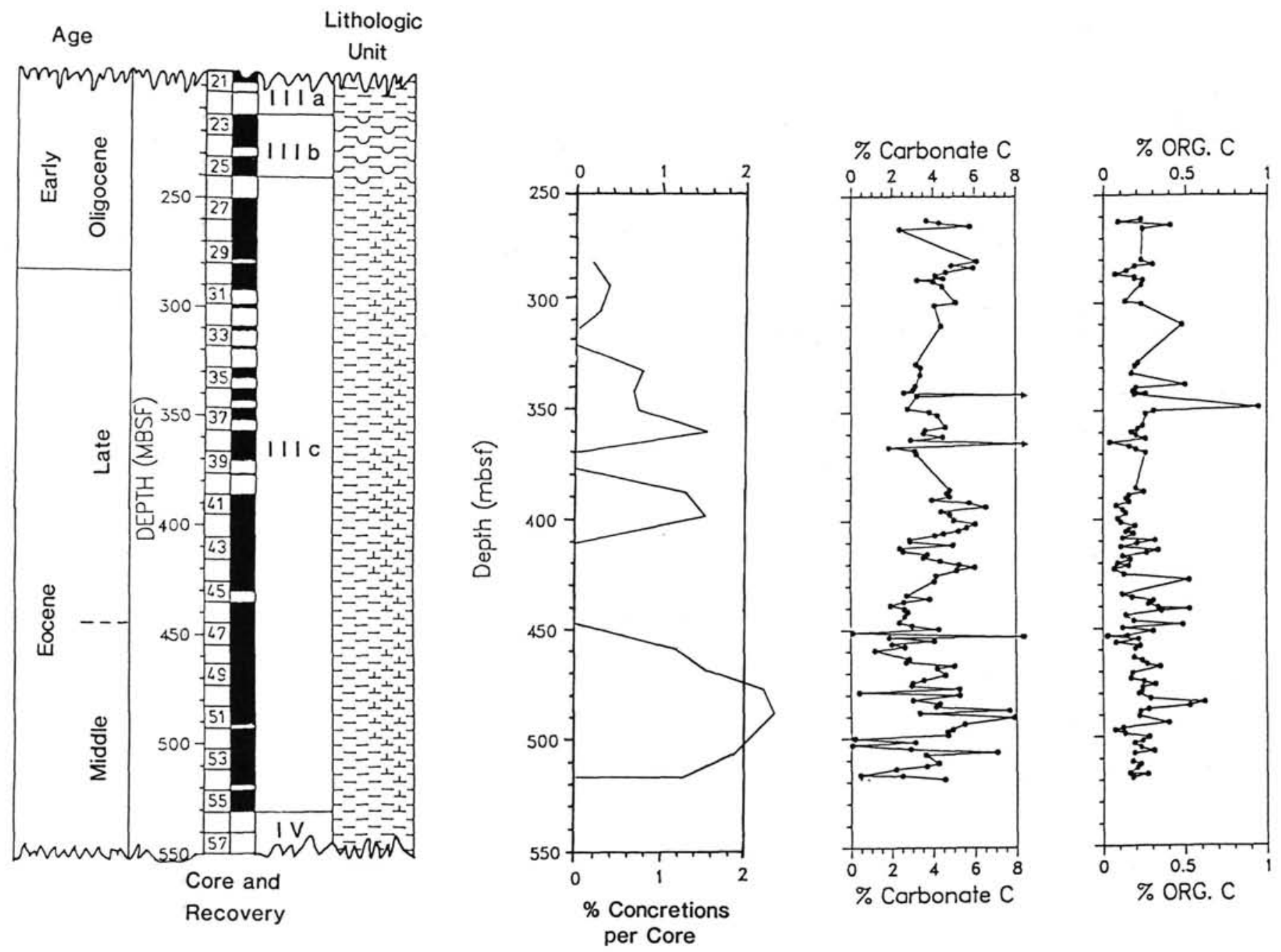

Figure 1. Age and lithology with depth for part of ODP Site 647 analyzed here. Percentage of concretionary layers per core (see Table 4), percent of carbonate carbon, and percent of organic carbon data from this study also are shown.

\section{RESULTS}

\section{Carbon and Carbonate Data}

Concentrations of organic carbon $(\mathrm{OC})$ and carbonate carbon (CC) are shown plotted vs. depth, core recovery, age, and lithologic units in Figure 1. The scale is the same as that of the geochemical plots that follow (Figs. 2 through 7). The samples cover the middle Eocene through lower Oligocene interval, encompassing lithologic Unit IIIC but not including the biogenic silica-rich interval that constitutes lithologic Unit IIIB.

The OC content of most samples is low, below $0.5 \mathrm{wt} \%$, and the average for lithologic Unit IIIB is about $0.25 \mathrm{wt} \%$. The CC is highly variable (multiply CC by 8.33 to obtain approximate $\mathrm{CaCO}_{3}$ content; see first column, Fig. 2, and last column, Table 1 ), and highest values correspond to intervals in which carbonate nodules or concretionary layers occur. The relative abundance of such intervals also is plotted in Figure 1.

\section{Inorganic Geochemistry}

The concentrations of major-element oxides plotted in Figure 2 are from XRF analyses, except for the five samples having high concentrations of $\mathrm{Fe}$ and $\mathrm{Mn}$, for which no XRF analyses exist (Table 1). There are no $\mathrm{SiO}_{2}$ results for these five samples, but the $\mathrm{SiO}_{2}$ concentrations are estimated at $10 \%$ to $12 \%$, based on the $\mathrm{Al}_{2} \mathrm{O}_{3}$ concentrations and the $\mathrm{SiO}_{2} / \mathrm{Al}_{2} \mathrm{O}_{3}$ ratios of adja- cent samples. Trace elements are grouped in Figures 3 through 6 , according to similarities of patterns of the downhole plots. The concentration plots for chromium $(\mathrm{Cr})$, lithium ( $\mathrm{Li}$ ), vanadium (V), scandium ( $\mathrm{Sc}$ ), and lead (Pb) (Fig. 3) all have similar patterns that are also similar to those of the major elements representative of a detrital fraction (e.g., $\mathrm{Al}_{2} \mathrm{O}_{3}$ and $\mathrm{TiO}_{2}$, Fig. 2), indicating that these trace elements are primarily associated with the clastic (clay) fraction. The concentration plots of the rare-earth elements (REE) cerium (Ce), lanthanum (La), neodymium (Nd), yttrium (Y), and ytterbium (Yb) (Fig. 4) are similar to one another and resemble the downhole plot of $\mathrm{P}_{2} \mathrm{O}_{5}$ (Fig. 2 ), suggesting that the REEs mainly are associated with one or more phosphate minerals, at least where the peaks of higher concentration are concerned.

The downhole plot of strontium (Sr) concentration (Fig. 5) parallels that of $\mathrm{CaO}$ (Fig. 2), indicating that $\mathrm{Sr}$ probably was substituted for $\mathrm{Ca}$ in one or more Ca-bearing mineral phases. However, the $\mathrm{Sr}$ and $\mathrm{CaO}$ concentrations do not always correlate with those in the downhole plot of $\mathrm{CaCO}_{3}$ (Fig. 1). The concentration of $\mathrm{CaCO}_{3}$ was calculated by assuming that all of the $\mathrm{CC}$ was derived from $\mathrm{CaCO}_{3}\left(\mathrm{CaCO}_{3}=\mathrm{CC} \times 8.33\right)$. However, as we discuss later, this assumption is not always true for the present set of samples. In most other data sets of carbonaterich, deep-sea sediments and rocks that we have analyzed (e.g., Dean and Parduhn, 1984; Arthur et al., 1987), the concentra- 

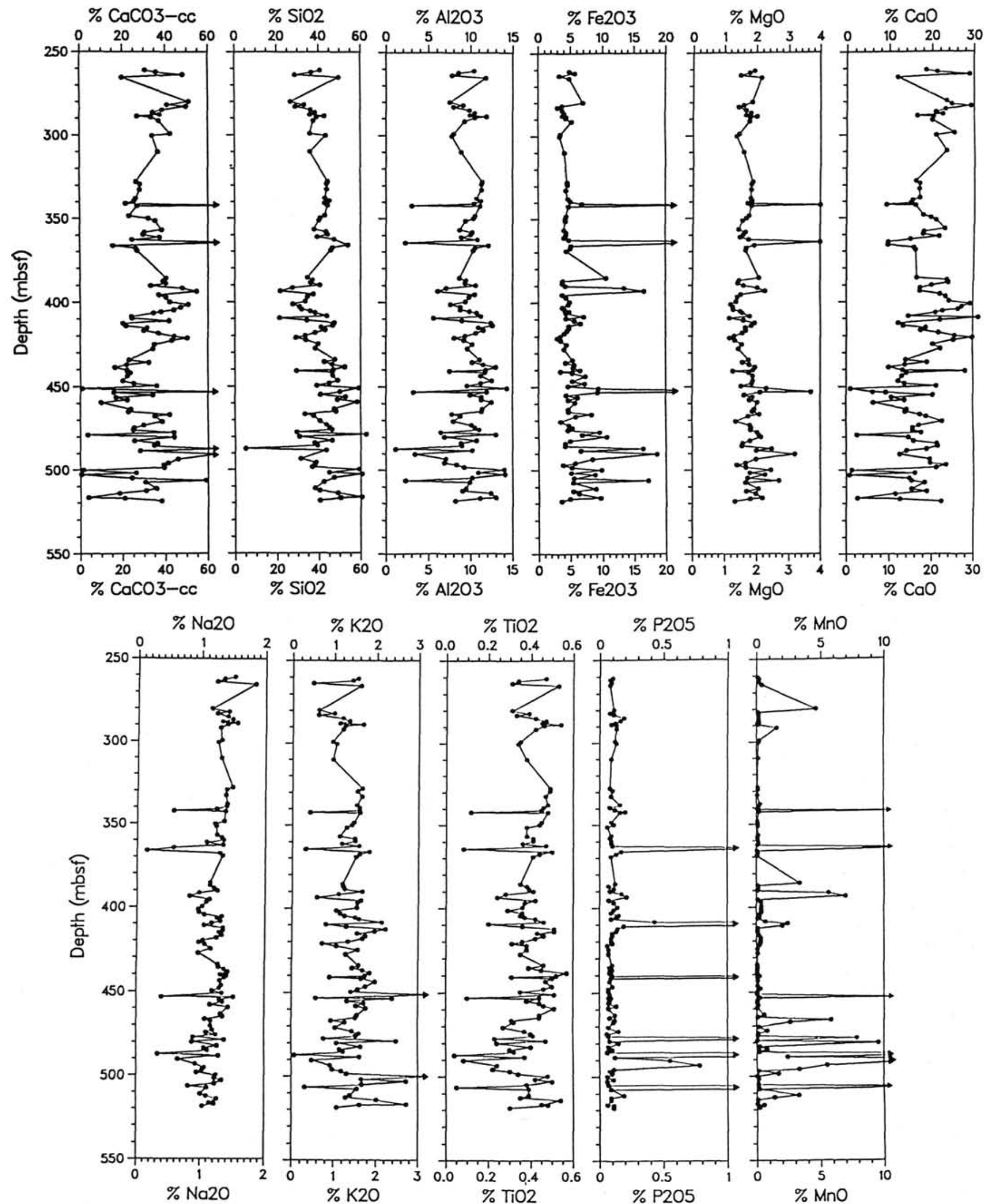

Figure 2. Concentrations of $\mathrm{CaCO}_{3}$ computed from carbonate carbon (CC) and major-element oxides in samples from lithologic Unit IIIC, Hole 647A. 

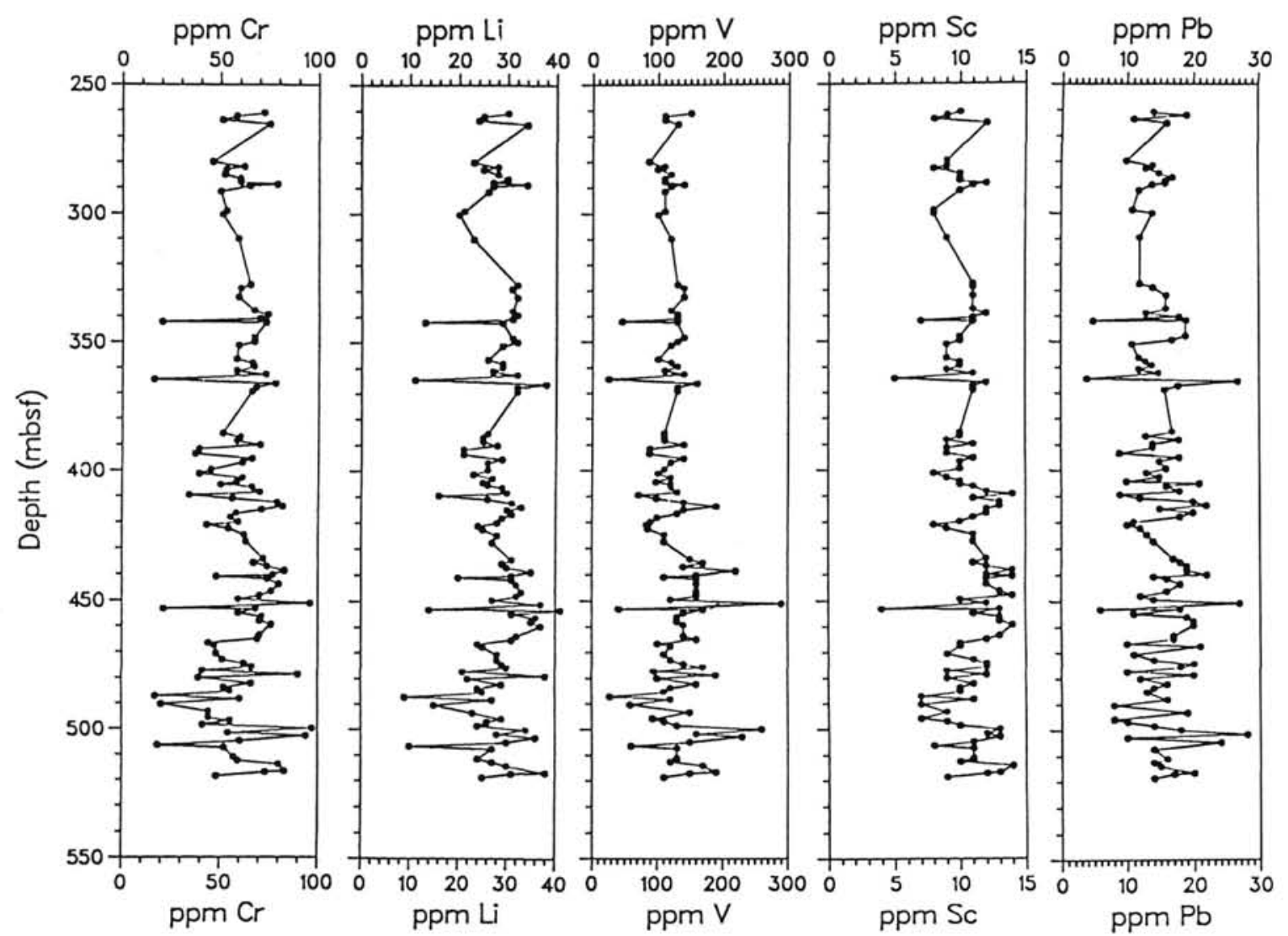

Figure 3. Concentrations of $\mathrm{Cr}, \mathrm{Li}, \mathrm{Sc}$, and $\mathrm{Pb}$ in samples from lithologic Unit IIIC, Hole 647A.

tion of $\mathrm{CaCO}_{3}$ also can be calculated from the total Ca concentration $\left(\mathrm{CaCO}_{3}=\mathrm{Ca} \times 2.5\right)$ with excellent agreement using $\mathrm{CaCO}_{3}$ concentration calculated from the $\mathrm{CC}$ concentration. Again, this assumption does not always work for the Site 647 samples.

Trace elements having downhole concentration patterns that are not obviously related to those of any other trace, minor, or major element include barium $(\mathrm{Ba})$, thorium $(\mathrm{Th})$, gallium $(\mathrm{Ga})$, niobium $(\mathrm{Nb})$, cobalt $(\mathrm{Co})$, copper $(\mathrm{Cu})$, nickel $(\mathrm{Ni})$, and zinc ( $\mathrm{Zn}$ ) (Figs. 5 and 6).

To examine more objectively the relationships among elements and to determine groupings of samples based on their chemistry, we performed a Q-mode factor analysis using the extended CABFAC computer program of Klovan and Miesch (1976). Before conducting the factor analysis, concentrations of all oxides and elements were transformed to proportions of the total range for each oxide and element. As a result of the transformation, all data were expressed on a scale of 0.0 to 1.0. After trying several different sets of reference axes in multidimensional space and different numbers of reference axes, we finally chose four orthogonal reference axes that maximize the variance of the transformed data in each dimension (four-factor varimax solution). The four-factor model accounted for more than $60 \%$ of the variance in the scaled data for 22 elements, with an average of $92 \%$. The four-factor model explained less than $30 \%$ of the variance in the scaled data for $\mathrm{Ba}, \mathrm{Co}, \mathrm{Cu}, \mathrm{Ga}, \mathrm{Nb}, \mathrm{Ni}, \mathrm{Th}$, and $\mathrm{Zn}$, which are the same elements that have unique downhole concentration patterns (Figs. 5 and 6). These eight elements were not included in the final Q-mode analysis. Basically, the four-factor Q-mode model reduced 22 measured variables (element concentrations plus LOI) to four "composite" geochemical variables. The intensities of the composite geochemical vari- ables are the factor loadings. The loadings for each of the four factors are plotted vs. depth below seafloor in Figure 7.

The factor loadings describe the relative importance of each composite chemical variable (factor) for each sample, but give no indication of which elements had the most influence on determining each of the four factors. For example, Samples 43-425 (409.55 mbsf), 46-5-111 (440.91 mbsf), 51-4-68 (487.28 mbsf), and 53-4-79 (506.45 mbsf) have the highest loadings for factor II, and these samples are clearly different from all other samples, but the loadings do not indicate what actual geochemical characteristics (element concentrations) had the most influence on distinguishing these samples from the others. To determine which elements had the most influence on which factor, the factor loadings for each sample were treated as composite chemical variables, and correlation coefficients were computed between the loadings and the 23 observed compositional variables. Results of the correlation analysis are given in Table 2 .

Table 2 shows that the elements that had the most influence on grouping samples in factor $\mathrm{I}$ are $\mathrm{Al}, \mathrm{Si}, \mathrm{Cr}, \mathrm{Ti}, \mathrm{K}, \mathrm{V}, \mathrm{Li}, \mathrm{Pb}$, $\mathrm{Sc}$, and $\mathrm{Na}$, in that order of importance (i.e., order of decreasing correlation coefficients). These are the elements that typically have a lithophile association (e.g., Goldschmidt, 1954); therefore, samples with high loadings for factor I have the greatest relative abundance of detrital clastic material.

Factor II is distinctly dominated by four samples, with moderate loadings for a few additional samples (Fig. 7), all in the lower one-half of lithologic Unit IIIC. Factor II samples are characterized by high concentrations of phosphorus and the REEs of $\mathrm{Nd}, \mathrm{Y}, \mathrm{Ce}, \mathrm{Yb}$, and La (Table 2).

Figure 7 shows that most samples have high loadings for factor III, which is the carbonate factor determined mainly by concentrations of $\mathrm{Ca}$ and $\mathrm{Sr}$ (Table 2). Factor IV, like factor II, is 


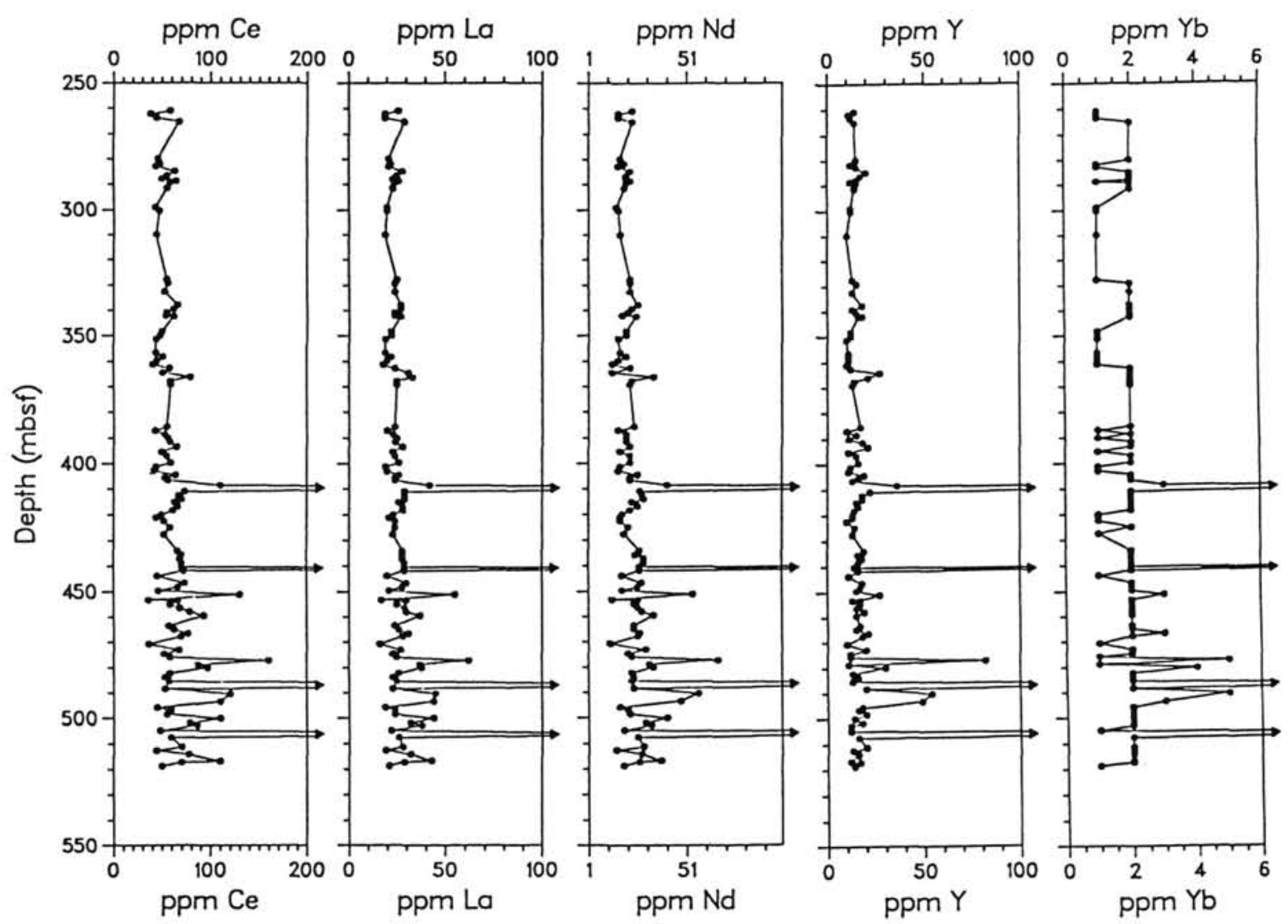

Figure 4. Concentrations of $\mathrm{Ce}, \mathrm{La}, \mathrm{Nd}, \mathrm{Y}$, and $\mathrm{Yb}$ in samples from lithologic Unit IIIC, Hole 647A.

dominated by four samples (although not the same four samples), with moderate loadings for a few other samples. The most important variables used to group factor IV samples are Mn, $\mathrm{Fe}, \mathrm{Mg}$, and $\mathrm{LOI}$.

The raw data plots (Figs. 1 through 6), particularly the plots of factor loadings (Fig. 7), show that most samples from lithologic Unit IIIC consist mainly of detrital clastic material plus varying amounts of $\mathrm{CaCO}_{3}$. That the clastic fraction is fairly typical of average crustal material is shown by Table 3, which compares the carbonate-free concentrations of elements in a typical sample from Unit IIIC (Sample 36-4-68) with those in average crust and average deep-sea clay. The most striking features of the downhole plots, however, are the horizons having distinctly different chemical characteristics because of high concentrations of one or more REE-bearing phosphate minerals and/or high concentrations of one or more $\mathrm{Mn}-$, Fe-, and $\mathrm{Mg}$ bearing minerals. Results of the $\mathrm{Ca}$ and $\mathrm{CC}$ analyses show that the phosphate mineral is indeed calcium phosphate (carbonatefluorapatite as determined by X-ray diffraction [XRD], Tables 4 and 5) and the Mn-, Fe-, and Mg-bearing minerals are one or more phases of carbonate, which XRD analysis confirmed was rhodochrosite, siderite, and manganosiderite. We mentioned earlier that for most deep-sea carbonate sediments or rocks we obtain essentially identical results for the percentage of $\mathrm{CaCO}_{3}$ calculated from total $\mathrm{Ca}$ and from CC. For the Site 647 samples, however, neither method gives correct results for all samples because of excess $\mathrm{Ca}$ from the apatite and excess $\mathrm{CC}$ from $\mathrm{Mn}-\mathrm{Fe}-\mathrm{Mg}$ carbonate. The difference between the two methods is shown in Figures 8 and 9. Figure 9 is a downhole plot of $\mathrm{CaCO}_{3}$ computed from $\mathrm{CC}-\mathrm{CaCO}_{3}$ computed from $\mathrm{Ca}$. The differences plotted in Figure 9 and the analytical results can be used to calculate the amount of apatite present, and the amount and molar proportions of cations in the $\mathrm{Mn}-\mathrm{Fe}-\mathrm{Mg}$ carbonate. For example, Sample 43-4-25 (409.55 mbsf) contains $31.3 \%$ $\mathrm{CaO}(=22.4 \% \mathrm{Ca}), 14.8 \% \mathrm{P}_{2} \mathrm{O}_{5}(=6.45 \% \mathrm{P})$, and $2.9 \% \mathrm{CC}$. Assuming that all of the $\mathrm{P}$ is in simple calcium fluorapatite $\left(\mathrm{Ca}_{5} \mathrm{~F}\left[\mathrm{PO}_{4}\right]_{3}\right)$ with a molar $\mathrm{Ca}: \mathrm{P}$ ratio of 1.67 , then $14.0 \% \mathrm{Ca}$ is in apatite, and the amount of apatite in the sample is $35 \%$. If all of the residual $\mathrm{Ca}(8.4 \%)$ is assumed to be in calcite, then the computed percentage of $\mathrm{CaCO}_{3}$ is $21 \%$, which compares with $24 \%$ computed from CC.

As an example of a $\mathrm{Mn}-\mathrm{Fe}-\mathrm{Mg}$ carbonate, Sample 38-6-67 (364.6 mbsf) contains $31.5 \% \quad \mathrm{Fe}_{2} \mathrm{O}_{3}(22 \% \mathrm{Fe}), 12.9 \% \mathrm{MnO}$ $(10 \% \mathrm{Mn}), 6.9 \% \mathrm{CaO}(6.9 \% \mathrm{Ca}), 1.6 \% \mathrm{P}_{2} \mathrm{O}_{5}(0.7 \% \mathrm{P})$, and $8.55 \%$ CC. Assuming that all of the $\mathrm{P}$ is in apatite with a $\mathrm{Ca}: \mathrm{P}$ ratio of 1.67 , and all of the residual $\mathrm{Ca}$ and all of the $\mathrm{Mg}, \mathrm{Mn}$, and $\mathrm{Fe}$ are in one carbonate mineral, then the formula for this carbonate mineral is:

$$
\left(\mathrm{Fe}_{0.52}, \mathrm{Mn}_{0.24}, \mathrm{Ca}_{0.17}, \mathrm{Mg}_{0.07}\right) \mathrm{CO}_{3} \text {. }
$$

Most of the other Fe- and Mn-rich samples have similar molar proportions of the four divalent cations.

The occurrence of these apparently authigenic carbonate and phosphate minerals seems anomalous for a primarily pelagic sedimentary sequence. However, the relatively high sedimentation rate (about $36 \mathrm{~m} / \mathrm{m}$.y.) over part of the sequence is more indicative of a hemipelagic regime, one in which suboxic to anoxic early diagenesis could induce substantial redistribution of organic-associated and redox-sensitive elements. In addition, there 

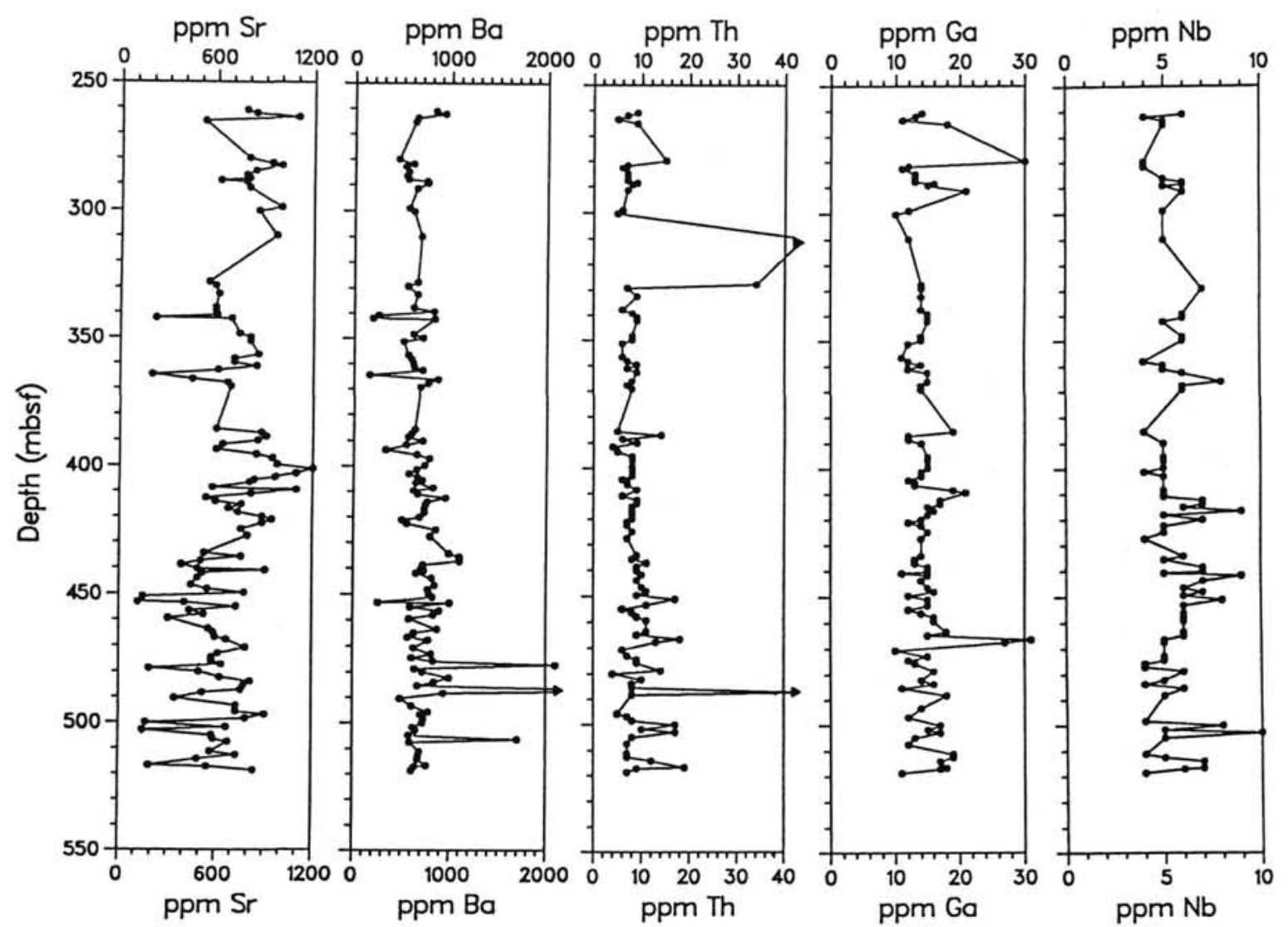

Figure 5. Concentrations of $\mathrm{Sr}, \mathrm{Ba}, \mathrm{Th}, \mathrm{Ga}$, and $\mathrm{Nb}$ in samples from lithologic Unit IIIC, Hole 647A.

is a potential for hydrothermal geochemical influx, at least in the lower part of the sequence. These possibilities are examined further in a later section of this chapter. Unfortunately, it is not possible to sort out the effects of diagenesis on primary geochemical signals related to paleoenvironment.

\section{X-Ray Diffraction Results}

Selected bulk samples, which had anomalous geochemical characteristics and which corresponded to levels of nodular or concretionary mineral layers, were analyzed using conventional XRD techniques (Table 5), as were a few of the "normal background" samples. The results generally confirm our inferences based on the geochemical data-specifically the elements $\mathrm{Ca}$, $\mathrm{Fe}, \mathrm{Mn}, \mathrm{Mg}, \mathrm{P}$, and inorganic $\mathrm{C}$. The most abundant authigenic carbonate phase is siderite or manganosiderite, typically characterized by broad peaks (Fig. 10), suggesting a phase intermediate in composition between siderite and rhodochrosite. No attempt was made to examine samples for compositional zoning because bulk sediment samples were ground for analysis. The broadened peaks possibly represent a number of discrete phases, with earliest precipitates having higher Mn contents and later precipitates having nearly pure siderite, as observed for some concretions from lower in the Eocene by Bohrmann and Thiede (this volume). Some samples consist of nearly pure siderite, but there appears to be no significant downcore trend in mineral dominance (Tables 4 and 5). In Cores 112-647A-43 and -46, nearly pure carbonate-fluorapatite concretions were detected on the basis of XRD (Fig. 10) and the anomalously high phosphate concentrations.

\section{Stable Isotope Results}

\section{Oxygen Isotopes}

Carbon and oxygen isotopic analyses were conducted on several species of benthic and planktonic foraminifers from a sequence at Site 647 spanning the late Eocene to early Oligocene. Results of these analyses are shown in Tables 6 and 7. Table 6 is a tabulation of visual preservation and abundance data for the samples selected for foraminifers.

Oxygen isotope values of planktonic foraminifers Globigerina eoceana and Catapsydrax unicavus exhibit a slight enrichment of about $0.5 \%$ in values of $\delta^{18} \mathrm{O}$ from the middle Eocene to early Oligocene. $G$. eoceana has $\delta^{18} \mathrm{O}$ values ranging from -1.80 to $-0.84 \%$, and $C$. unicavus is characterized by values of -1.84 to $-0.54 \%$. These $\delta^{18} \mathrm{O}$ values (Fig. 11) are in agreement with the oxygen isotope values of mixed planktonic foraminifers of the same age from nearby Site 112 (W. Curry and K. Miller, pers. comm., 1989). Similar but slightly greater enrichments have been reported at numerous other Eocene/Oligocene sequences worldwide and have been interpreted to represent either a global cooling of several degrees or an expansion of continental ice volume (e.g., Shackleton and Kennett, 1975; Keigwin, 1980; Keigwin and Corliss, 1986; Miller et al., 1987; Boersma et al., 1987).

No consistent difference exists in $\delta^{18} \mathrm{O}$ between $G$. eoceana and $C$. unicavus at Site 647 , in contrast to what was observed in low- to mid-latitude sequences (Keigwin and Corliss, 1986). This suggests either that the species maintained similar depth habitats, or as is the case for high latitudes in the modern ocean, the vertical temperature gradient in Labrador Sea surface waters 


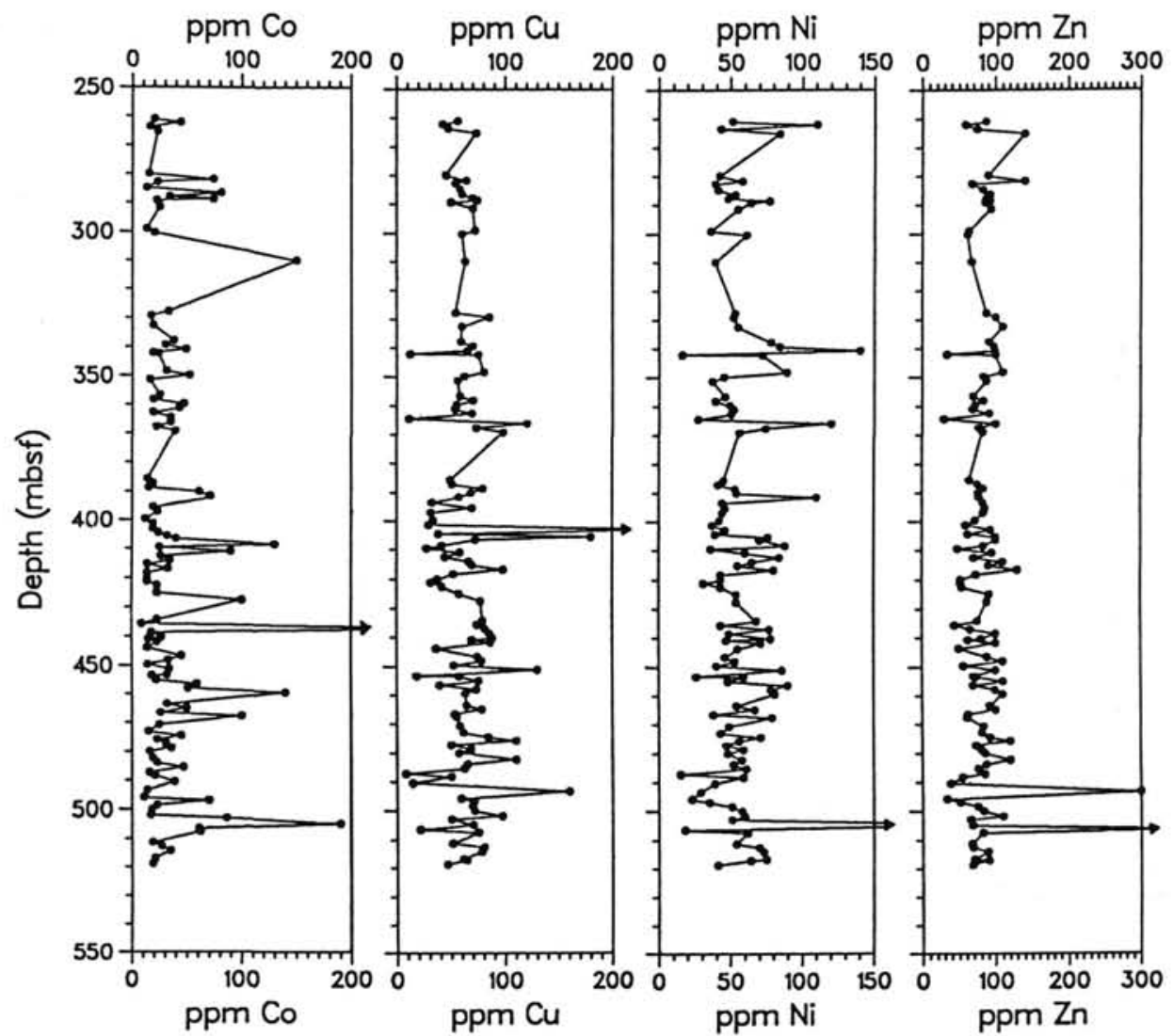

Figure 6. Concentrations of $\mathrm{Co}, \mathrm{Cu}, \mathrm{Ni}$, and $\mathrm{Zn}$ in samples from lithologic Unit IIIC, Hole 647A.

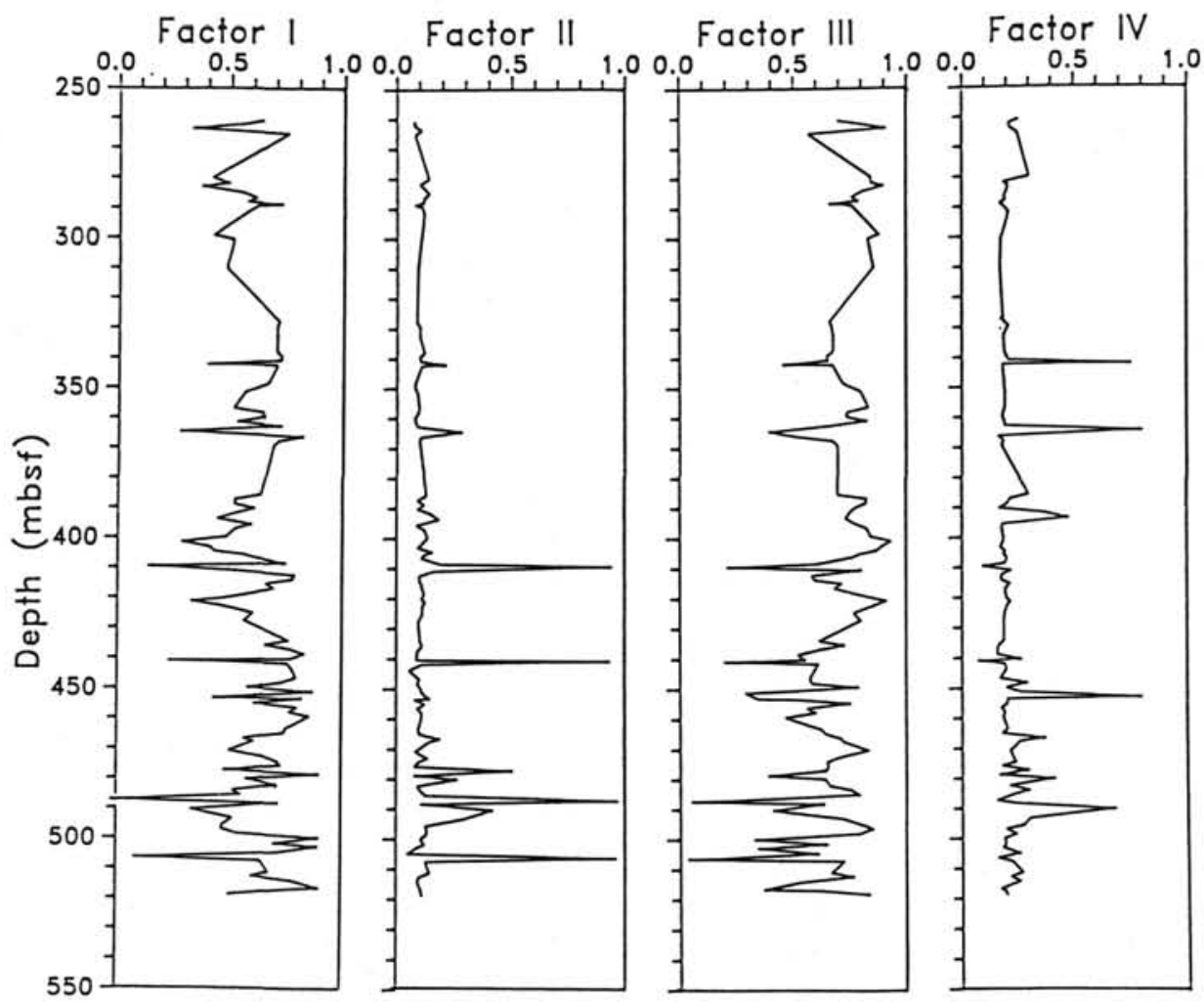

Figure 7. Plots of factor loadings from a four-factor Q-mode factor analysis of element concentrations in samples from lithologic Unit IIIC, Hole 647A. 
Table 2. Correlations between the factor loadings and the variables.

\begin{tabular}{|c|c|c|c|c|}
\hline & I & II & III & IV \\
\hline $\mathrm{SiO}_{2} \%$ & 0.8882 & -0.6438 & -0.1195 & -0.1665 \\
\hline $\mathrm{Al}_{2} \mathrm{O}_{3} \%$ & 0.8967 & -0.6939 & -0.0489 & -0.1917 \\
\hline $\mathrm{Fe}_{2} \mathrm{O}_{3} \%$ & -0.2113 & 0.1085 & -0.2944 & 0.9155 \\
\hline $\mathrm{MgO} \%$ & -0.0893 & 0.0166 & -0.2915 & 0.9087 \\
\hline $\mathrm{CaO} \%$ & -0.5696 & -0.1478 & 0.7765 & 0.2042 \\
\hline $\mathrm{Na}_{2} \mathrm{O} \%$ & 0.6597 & -0.6406 & 0.0656 & 0.0581 \\
\hline $\mathrm{K}_{2} \mathrm{O} \%$ & 0.8468 & -0.4933 & -0.2978 & -0.1563 \\
\hline $\mathrm{TiO}_{2} \%$ & 0.8524 & -0.6825 & 0.0196 & -0.2559 \\
\hline $\mathrm{P}_{2} \mathrm{O}_{5} \%$ & -0.5525 & 0.8537 & -0.5492 & 0.0547 \\
\hline $\mathrm{MnO} \%$ & -0.3972 & 0.3067 & -0.2944 & 0.9506 \\
\hline LOI $900^{\circ} \mathrm{C}$ & -0.4364 & -0.1170 & 0.3408 & 0.8316 \\
\hline Ce ppm-s & -0.4014 & 0.8924 & -0.7525 & 0.0458 \\
\hline Cr ppm-s & 0.8570 & -0.6365 & -0.1236 & -0.1810 \\
\hline La ppm-s & -0.3893 & 0.8281 & -0.6882 & 0.0778 \\
\hline $\mathrm{Li}$ ppm-s & 0.7828 & -0.6665 & -0.0816 & 0.1197 \\
\hline Nd ppm-s & -0.4244 & 0.9300 & -0.7817 & 0.0106 \\
\hline $\mathrm{Pb}$ ppm-s & 0.7718 & -0.5601 & -0.0733 & -0.0771 \\
\hline Sc ppm-s & 0.7054 & -0.4589 & -0.2454 & 0.2433 \\
\hline Sr ppm-s & -0.4405 & -0.2691 & 0.8864 & -0.0989 \\
\hline V ppm-s & 0.8234 & -0.5115 & -0.2417 & -0.1494 \\
\hline Y ppm-s & -0.5560 & 0.9280 & -0.6184 & 0.0519 \\
\hline Yb ppm-s & -0.4786 & 0.8808 & -0.6685 & 0.2118 \\
\hline
\end{tabular}

Table 3. Concentrations of major-element oxides and trace elements in average upper continental crust (Wedepohl, 1971), average deep-sea clay (Chester and Aston, 1976), red clay from the Bermuda Rise (V26157), carbonate-free Sample $105647 \mathrm{~A}-36 \mathrm{R}-4,68 \mathrm{~cm}$, and average background sediments $(n=99)$ from Site 647 .

\begin{tabular}{|c|c|c|c|c|c|}
\hline & Avg. Crust & $\begin{array}{c}2 \\
\text { Avg. } \\
\text { Deep-Sea } \\
\text { Clay }\end{array}$ & V26-157 & $\begin{array}{c}4 \\
36-4-68 \\
\text { (carb.-free) }\end{array}$ & $\begin{array}{c}5 \\
\text { Avg. Bkgrd. } \\
(\mathrm{n}=99) \\
\text { (carb.-free) }\end{array}$ \\
\hline \multicolumn{6}{|l|}{ Oxide $(\%)$} \\
\hline $\mathrm{SiO}_{2}$ & 65.3 & 60.4 & 53.5 & 62.4 & 62.1 \\
\hline $\mathrm{Al}_{2} \mathrm{O}_{3}$ & 14.8 & 19.1 & 18.9 & 15.6 & 15.2 \\
\hline $\mathrm{Fe}_{2} \mathrm{O}_{3}$ & 5.06 & 8.93 & 8.15 & 6.6 & 7.3 \\
\hline $\mathrm{MgO}^{3}$ & 2.30 & 3.73 & 2.88 & 2.6 & 2.5 \\
\hline $\mathrm{Na}_{2} \mathrm{O}$ & 3.29 & 1.67 & 2.02 & 1.9 & 1.9 \\
\hline $\mathrm{K}_{2} \mathrm{O}$ & 3.38 & 3.56 & 3.96 & 2.3 & 2.2 \\
\hline $\mathrm{TiO}_{2}$ & 0.71 & 0.92 & 0.84 & 0.68 & 0.61 \\
\hline $\mathrm{P}_{2} \mathrm{O}_{5}$ & 0.19 & 0.16 & 0.16 & 0.23 & 0.17 \\
\hline $\mathrm{MnO}$ & 0.09 & 0.52 & 0.14 & 0.27 & 0.60 \\
\hline \multicolumn{6}{|c|}{ Element (ppm) } \\
\hline $\mathrm{Ba}$ & 590 & 2300 & 503 & 1170 & 1050 \\
\hline Co & 12 & 74 & 26 & 34 & 58 \\
\hline $\mathrm{Cr}$ & 70 & 90 & 89 & 100 & 94 \\
\hline $\mathrm{Cu}$ & 30 & 250 & 42 & 110 & 100 \\
\hline $\mathrm{Ga}$ & 17 & 20 & 31 & 21 & - \\
\hline $\mathrm{La}$ & 44 & 115 & 50 & 38 & 38 \\
\hline $\mathrm{Li}$ & 30 & 57 & 94 & 41 & 43 \\
\hline $\mathrm{Nb}$ & & 14 & - & 7 & 8 \\
\hline $\mathrm{Nd}$ & & 140 & - & 35 & 34 \\
\hline $\mathrm{Ni}$ & 44 & 225 & 58 & 100 & 89 \\
\hline $\mathrm{Pb}$ & 15 & 80 & 20 & 27 & 23 \\
\hline $\mathrm{Sc}$ & 14 & 19 & 19 & 15 & 16 \\
\hline Th & 11 & 5 & - & 13 & 13 \\
\hline V & 60 & 120 & 127 & 180 & 200 \\
\hline $\mathrm{Y}$ & 34 & 9 & 27 & 23 & 22 \\
\hline $\mathrm{Yb}$ & & 15 & - & 3 & 2.5 \\
\hline $\mathrm{Zn}$ & 60 & 165 & 120 & 140 & 130 \\
\hline
\end{tabular}

was minimal at this time. Converting the oxygen isotope values to paleotemperature would indicate a range of $16^{\circ}$ to $20^{\circ} \mathrm{C}$ for the Labrador Sea, depending on whether ice-free conditions are assumed. Although broken shells are common, visual and scanning electron microscope (SEM) examination showed that the preservation of the planktonic foraminifer shells was good, with little or no sign of infilling and/or external overgrowth.
Table 4. Abundance of concretionary intervals in Cores 105-647C-28 through -54, estimated from core photographs.

\begin{tabular}{|c|c|c|c|c|}
\hline $\begin{array}{c}\text { Core } \\
\text { number }\end{array}$ & $\begin{array}{l}\text { No. of } \\
\text { concretion } \\
\text { zones }\end{array}$ & $\begin{array}{l}\text { Core } \\
\text { recovery } \\
\text { (m) }\end{array}$ & $\begin{array}{l}\text { Avg. and total } \\
\text { thickness } \\
\text { concretionary } \\
\text { zone }\end{array}$ & $\begin{array}{c}\text { Sediment } \\
\text { that is } \\
\text { concretion } \\
(\%)\end{array}$ \\
\hline 29 & 0 & 6.0 & - & 0 \\
\hline 30 & 2 & 9.5 & $(1 \mathrm{~cm}) 2$ & 0.22 \\
\hline 31 & 1 & 2.7 & $(1 \mathrm{~cm}) 1$ & 0.37 \\
\hline 32 & 1 & 2.0 & $(0.5 \mathrm{~cm}) 0.5$ & 0.25 \\
\hline 33 & 0 & 2.4 & - & 0 \\
\hline 34 & 0 & 1.5 & - & 0 \\
\hline 35 & 1 & 5.2 & $(4.0 \mathrm{~cm}) 4$ & 0.77 \\
\hline 36 & 2 & 6.0 & $(2 \mathrm{~cm}) 4$ & 0.67 \\
\hline 37 & 2 & 5.65 & $(2 \mathrm{~cm}) 4$ & 0.70 \\
\hline 38 & 5 & 9.5 & $(3 \mathrm{~cm}) 15$ & 1.60 \\
\hline 39 & 0 & 4.0 & - & 0 \\
\hline 40 & 0 & 0.25 & - & 0 \\
\hline 41 & 6 & 8.7 & $(1.8 \mathrm{~cm}) 11$ & 1.30 \\
\hline 42 & 4 & 9.7 & $(3.8 \mathrm{~cm}) 15$ & 1.55 \\
\hline 43 & 0 & 9.5 & - & 0 \\
\hline 44 & 0 & 8.7 & - & 0 \\
\hline 45 & 0 & 4.3 & - & 0 \\
\hline 46 & 0 & 9.0 & - & 0 \\
\hline 47 & 0 & 8.0 & - & 0 \\
\hline 48 & 1 & 8.5 & $(10 \mathrm{~cm}) 10$ & 1.18 \\
\hline 49 & 7 & 9.5 & $(2.1 \mathrm{~cm}) 14.5$ & 1.53 \\
\hline 50 & 12 & 9.0 & $(1.6 \mathrm{~cm}) 19$ & 2.11 \\
\hline 51 & 13 & 9.0 & $(1.7 \mathrm{~cm}) 21.5$ & 2.39 \\
\hline 52 & 9 & 6.5 & $(1.5 \mathrm{~cm}) 13.5$ & 2.10 \\
\hline 53 & 9 & 7.2 & $(1.6 \mathrm{~cm}) 14$ & 1.94 \\
\hline 54 & 8 & 8.0 & $(1.25 \mathrm{~cm}) 10$ & 1.25 \\
\hline
\end{tabular}

In contrast to the planktonic foraminifers, benthic foraminifers from the lower Eocene to lower Oligocene interval yield a much wider range of $\delta^{18} \mathrm{O}$ values. Samples of Oridorsalis species and Globocassidulina subglobosa from about 285 mbsf near the Eocene/Oligocene boundary yield $\delta^{18} \mathrm{O}$ values of 0.28 to $0.72 \%$. These values are also similar to those recorded for Gyroidinoides species from early Oligocene cores of Site 112 (Curry and Miller, unpub. data) as well to values for benthic foraminifers of similar age from other Atlantic locations (Miller et al., 1987; Keigwin and Corliss, 1986). However, farther below, in the upper Eocene part of the sequence, a progressive downcore depletion occurs in the oxygen isotopic values of the benthic foraminifers. From 285 to $400 \mathrm{mbsf}$, the $\delta^{18} \mathrm{O}$ of both Oridorsalis species and Cibicidoides decrease by more than $4 \%$. Other species of benthic foraminifers, Nutallides truempeyi, G. subglobosa, and Stilostomella exhibit similar depletions, although not so large.

A puzzling aspect of these more negative oxygen-isotope values is that the $\delta^{18} \mathrm{O}$ values of planktonic foraminifers remain more-or-less constant throughout the interval in which the values of benthic foraminifers decline. If interpreted in terms of temperature, the negative $\delta^{18} \mathrm{O}$ gradient from surface to bottom would imply an inverted density gradient, with much warmer bottom waters. Because these environmental conditions seem unreasonable, differential diagenesis must be responsible for the observed isotopic trends. The depleted values of the benthic foraminifers indicate that recrystallization, or carbonate cementation, may have occurred within the benthic foraminiferal tests. Visual examination of the benthic foraminifers revealed some signs of carbonate overgrowths, but most samples are reasonably well-preserved (moderate to good). The planktonic foraminifers display few signs of cement overgrowth. Why such selective diagenesis occurred is unclear at this time. The tests of benthic foraminifers tend to be more robust and coarsely crystalline; whereas, tests of calcareous nannofossils and planktonic foraminifers are generally more porous, fine-grained, and solution susceptible. If substantial dissolution/recrystallization did oc- 
Table 5. Whole-sediment (concretions or layers) XRD results.

\begin{tabular}{|c|c|c|c|c|}
\hline $\begin{array}{c}\text { Core/section/ } \\
\text { interval }\end{array}$ & $\begin{array}{l}\text { Depth } \\
\text { (mbsf) }\end{array}$ & $\begin{array}{c}\text { Main minerals } \\
\text { present }\end{array}$ & Comments & $\mathrm{Fe}_{2} \mathrm{O}_{3}, \mathrm{MnO}, \mathrm{P}_{2} \mathrm{O}_{5}(\%)$ \\
\hline $105-647 \mathrm{~A}-30-1,51$ & 279.93 & Siderite-Rhodochrosite-Calcite & & (not anomalous) \\
\hline $36-4,42$ & 342.13 & Siderite-Manganosiderite & (broad peaks) & $(31.5,11.5,0.21)$ \\
\hline $38-6,67$ & 364.56 & Siderite-Manganosiderite & (broad peaks) & $(31.5,12.9,1.56)$ \\
\hline $43-4,25$ & 409.55 & Aparite-Calcite & & $(4.31,2.42,14.8)$ \\
\hline $46-5,111$ & 440.91 & Aparite & & $(3.46,0.23,11.3)$ \\
\hline $48-1,7$ & 453.27 & Siderite-Manganosiderite & (sharp peaks) & $(37.2,11.5,0.55)$ \\
\hline $50-4,32$ & 477.32 & Siderite-Rhodochrosite-Calcite & & $(9.5,7.86,2.01)$ \\
\hline $51-4,68$ & 487.28 & Rhodochrosite-Siderite & (broad peaks) & $(16.4,20.2,7.31)$ \\
\hline $51-6,83$ & 490.43 & Siderite-Rhodochrosite-Calcite & $\begin{array}{l}\text { (Calcite peak shift; } \\
\text { Mg substitution) }\end{array}$ & $(18.6,15.5,0.55)$ \\
\hline $52-2,10$ & 493.40 & Calcite-(minor) Siderite & & (not anomalous) \\
\hline $53-4,79$ & 506.45 & Manganosiderite & & $(17.2,16.7,7.6)$ \\
\hline
\end{tabular}

Note: Diffractometer parameters: $1.2^{\circ} 2 \theta / \mathrm{min}$ from 10 to $60^{\circ} 2 \theta$ with $\mathrm{CuK} \alpha$ radiation.

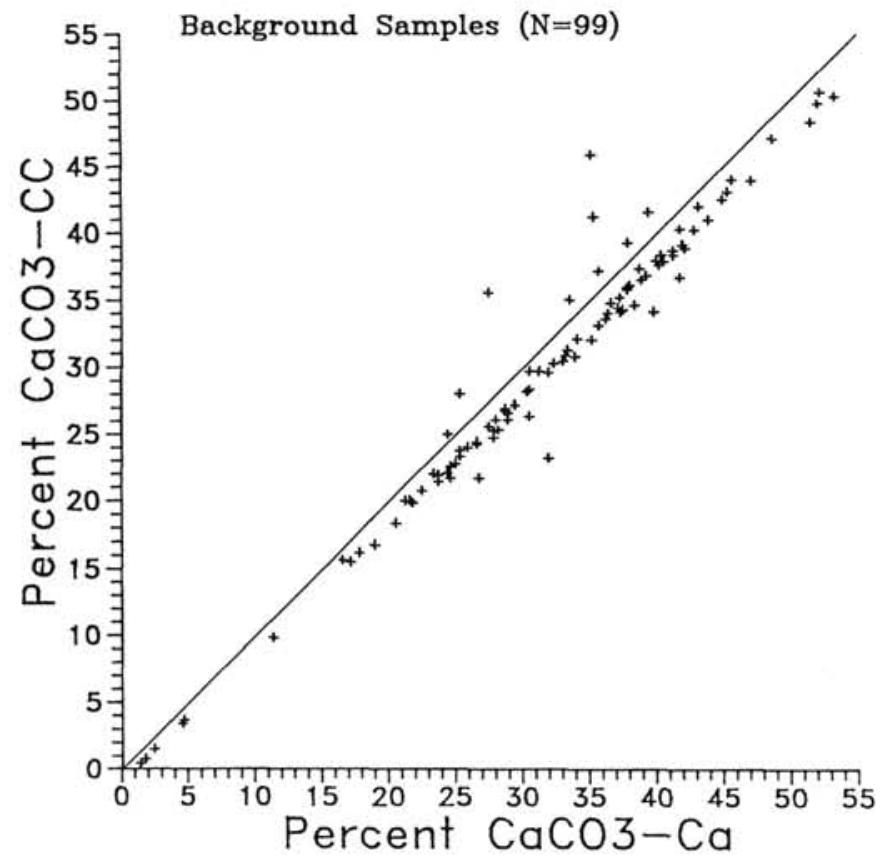

Figure 8. Plot of $\mathrm{CaCO}_{3}$ of "background" (nonconcretionary) samples from Hole 647A calculated from carbonate carbon (coulometry, see text) vs. that calculated from $\mathrm{Ca}$ geochemical data. Note close agreement between two techniques, with the exception of a few points, which probably contain authigenic carbonate phases.

cur, the solution-susceptible planktonic tests may be the primary source of carbonate, while the interior and exterior walls of benthic tests are the selective sites for cement precipitation. Of the benthic foraminifers, Oridorsalis species and Cibicidoides consistently display the lightest values, suggesting that of the benthics these species provide the most favorable sites for cement precipitation.

\section{Carbon Isotopes}

As with the oxygen isotopes, the carbon isotopic composition of the planktonic foraminifers is little affected by diagenesis. Samples of both $C$. unicavus and $G$. eoceana have $\delta^{13} \mathrm{C}$ values of 0.50 to $0.75 \%$ from the middle Eocene, again with no discernable difference between the species. A slight decrease in $\delta^{13} \mathrm{C}$ can be measured from the middle to upper Eocene parts of the seafloor, where the average values of planktonic foraminifers are less than $0.25 \%$ (Fig. 11).

The carbon isotopic composition of the benthic foraminifers is progressively more negative from lower to upper Eocene at

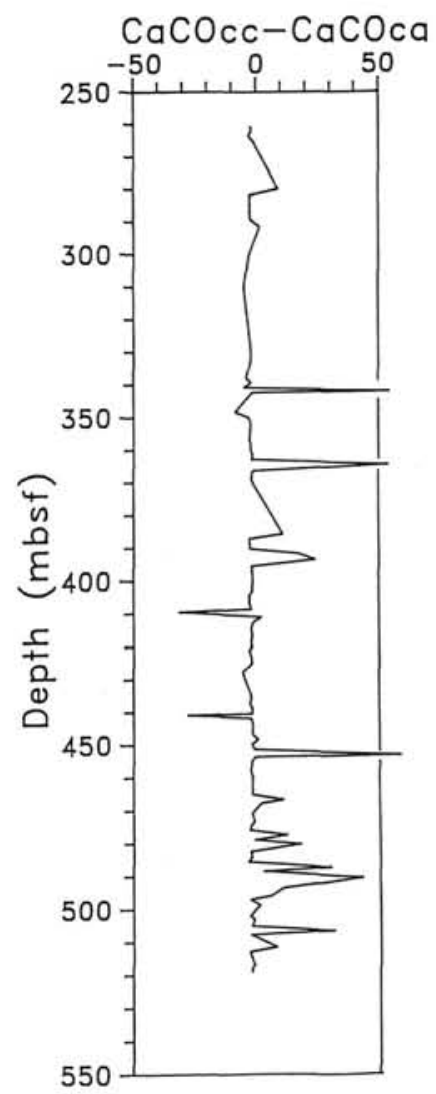

Figure 9. Plot of difference in $\mathrm{CaCO}_{3}$ computed from carbonate carbon (CC) and from calcium for samples from lithologic Unit IIIC, Hole 647A.

Site 647 (Fig. 11). The measured $\delta^{13} \mathrm{C}$ values for all species range from -0.7 to $+0.5 \%$ in the lower Eocene and from -2.0 to $-0.3 \% 0$ in the upper Eocene, with Oridorsalis consistently yielding the lightest values. The carbon isotopic compositions of $\mathrm{Nu}$ tallides and Cibicidoides overlap upsection and are on average $1.0 \%$ heavier than those of Oridorsalis.

Comparatively heavier $\delta^{13} \mathrm{C}$ values, in the range of -0.25 to $1.5 \%$, have been reported for various species of benthic foraminifers from other Eocene pelagic sequences located in the equatorial Pacific (Keigwin, 1980), south Atlantic, eastern Indian Arabian Sea (Keigwin and Corliss, 1986), and the Bay of Biscay (Miller et al., 1985). In addition, the records at these locations give no indication of a depletion in the deep-water mass carbon isotopic composition during the middle to late Eocene. 


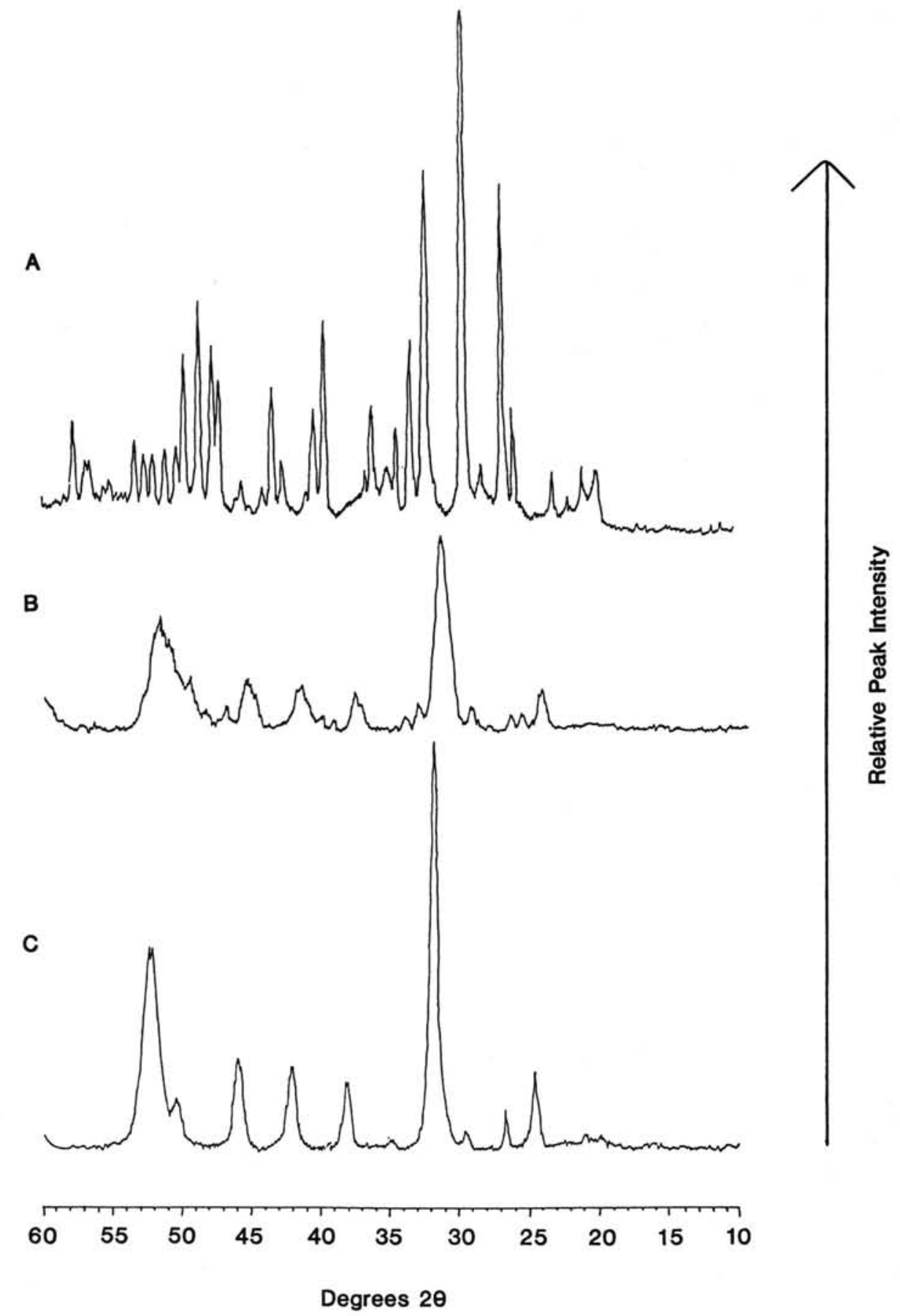

Figure 10. Representative X-ray diffractograms of three different concretionary phases. A. Sample 105-647A-46R-5, $111 \mathrm{~cm}$ : all sharp peaks indicate pure carbonate fluorapatite phase. B. Sample $105-647 \mathrm{~A}-51 \mathrm{R}-4,68 \mathrm{~cm}$ : broadened peaks suggest composition intermediate between rhodochrosite and siderite (i.e., manganosiderite) or a range of Fe-Mn carbonates in bulk samples. C. Sample 105-647A-48R-1, $7 \mathrm{~cm}$ : sharp peaks mainly siderite, with some manganese substitution, but no discrete rhodochrosite.

This discrepancy may arise from one of several factors as outlined below.

The interstitial waters of the upper few centimeters of the substrate at this particular location could have been characterized by substantially lighter carbon isotope compositions. Benthic foraminifers inhibiting shallow levels in the sediment could then have precipitated carbonate in equilibrium with a lighter total dissolved carbon source. This would have occurred if the amount of isotopically light $\mathrm{OC}$ accumulating and decaying within the substrate were higher here than elsewhere (e.g., McCorkle, 1987). Productivity (OC flux), sedimentation rate, and/ or the oxygen levels of the deep-water mass all have a bearing on the preservation of $\mathrm{OC}$ in the sediments. It is difficult to determine which of these parameters exerted the greatest influence of $\mathrm{OC}$ accumulation rates here, but sedimentation rate may have had the most influence. Neither carbonate nor biogenic opal ac- 
Table 6. Visual (microscopic) estimation of preservation of samples picked for benthic and planktonic foraminifers for stable-isotope analyses.

\begin{tabular}{|c|c|c|c|c|}
\hline $\begin{array}{l}\text { Core, section } \\
\text { interval }(\mathrm{cm})\end{array}$ & $\begin{array}{l}\text { Depth } \\
\text { (mbsf) }\end{array}$ & $\begin{array}{c}\text { Benthic } \\
\text { preservation }\end{array}$ & Comments & Planktonics \\
\hline $105-647 \mathrm{~A}-28-1,108-115$ & 261.18 & G & & \\
\hline $28-2,105-108$ & 262.65 & G & & \\
\hline $28-3,105-108$ & 264.15 & G & & \\
\hline $28-4,91-98$ & 265.51 & G & & \\
\hline $28-4,105-108$ & 265.65 & G & & \\
\hline $30-1,107-111$ & 280.47 & G & & Few dwarf \\
\hline $30-2,25-29$ & 281.15 & G & & G \\
\hline $30-3,110-114$ & 283.54 & G & & G \\
\hline $30-4,107-111$ & 284.97 & $\mathrm{M} / \mathrm{G}$ & (Few pits, pyrite) & Few \\
\hline $30-5,10-12$ & 285.50 & $\mathrm{M} / \mathrm{G}$ & (Some etching, Nuttalides abundant) & \\
\hline $30-7,33-38$ & 288.73 & $\mathrm{M} / \mathrm{G}$ & (Some pyrite in-Orid.) & $\mathrm{M} / \mathrm{G}$ \\
\hline $31-1,132-136$ & 299.92 & $\mathrm{M} / \mathrm{G}$ & (Signs of etching) & \\
\hline $31-2,34-37$ & 290.84 & $\mathrm{M} / \mathrm{G}$ & (Some etching) & \\
\hline $32-1,84-93$ & 299.44 & $\mathrm{M}$ & (Stronger etching, Orid./pyrite) & M \\
\hline $32-2,20-24$ & 300.30 & $M / G$ & (Some etching, Gyrodin.) & \\
\hline $33, \mathrm{CC}$ & 310.60 & $\mathrm{M} / \mathrm{G}$ & (No pyrite, some etching) & \\
\hline $34, \mathrm{CC}$ & 319.30 & $\mathrm{M} / \mathrm{G}$ & (No visible pyrite) & \\
\hline $35-1,77-80$ & 328.37 & $\mathrm{M} / \mathrm{G}$ & (Abund. pyrite on all forms) & \\
\hline $35-2,77-80$ & 329.87 & $\mathrm{M}$ & (Pyrite; etching; broken spec.) & $\mathrm{M} / \mathrm{G}$ \\
\hline $35-3,77-80$ & 331.37 & $\mathrm{M} / \mathrm{G}$ & (Better than above, no pyrite) & \\
\hline $36-2,49-52$ & 339.19 & M & (Abund. pyrite, etching) & $\begin{array}{l}\text { M low } \\
\text { abund. }\end{array}$ \\
\hline $36-3,49-52$ & 340.69 & $\mathrm{M} / \mathrm{G}$ & (No pyrite, better than above) & \\
\hline $36-4,49-52$ & 342.19 & $\mathrm{M} / \mathrm{G}$ & (Pyrite present) & \\
\hline $37-2,90-93$ & 349.10 & M & (Abund. pyrite) & \\
\hline $37-3,90-93$ & 350.60 & $\mathrm{P} / \mathrm{M}$ & (Abund. pyrite, etching frags.) & low abund. \\
\hline $37-4,90-93$ & 352.10 & $\mathrm{M} / \mathrm{G}$ & (Pyrite absent) & \\
\hline $38-1,83-86$ & 357.24 & $\mathrm{M}$ & (Orange; some etching) & M/G abund. \\
\hline $38-2,84-87$ & 358.74 & $M / G$ & (Orange) & \\
\hline $38-3,86-89$ & 360.26 & $\mathrm{M} / \mathrm{G}$ & (Clear/White) & \\
\hline $38-4,86-89$ & 361.76 & $\mathrm{M} / \mathrm{G}$ & (No pyrite/clear) & \\
\hline $39-1,80-83$ & 366.90 & M & (Orange/pyrite) & M \\
\hline $39-2,77-80$ & 368.37 & $\mathrm{M} / \mathrm{G}$ & (Low abund., trace pyrite) & (w/ppm) \\
\hline $39, \mathrm{CC}$ & 370.10 & $\mathrm{M}$ & (Orange; pyrite abund.) & M (some pyr.) \\
\hline $41-1,58-61$ & 385.98 & $\mathrm{M} / \mathrm{G}$ & (White, some pyrite) & \\
\hline $41-3,58-61$ & 288.98 & M & (White, no pyrite) & $\mathrm{M} / \mathrm{G}$ \\
\hline $41-5,58-61$ & 391.98 & $\mathrm{M} / \mathrm{G}$ & (White, no pyrite) & \\
\hline $42-3,32-35$ & 398.42 & $\mathrm{M} / \mathrm{G}$ & (White, no pyrite) & \\
\hline $42-5,96-100$ & 402.06 & $\mathrm{M} / \mathrm{G}$ & (White, no pyrite) & \\
\hline $43-1,95-98$ & 405.75 & $\mathrm{M}$ & (White, no pyrite) & \\
\hline $43-3,104-108$ & 408.84 & M & (Some etching, no pyrite, white) & $\mathrm{M} / \mathrm{G}$ \\
\hline $43-5,97-100$ & 411.77 & M & (Dissolved, some pyrite) & \\
\hline $44-1,45-48$ & 414.95 & $\mathrm{M} / \mathrm{G}$ & (Some pyrite) & $\mathrm{M} / \mathrm{G}$ \\
\hline $44-3,42-46$ & 417.92 & $\mathrm{M} / \mathrm{G}$ & (No pyrite) & \\
\hline $44-5,45-48$ & 420.95 & G & (No pyrite, white, clear) & \\
\hline $45-1,14-18$ & 424.24 & $\mathrm{M} / \mathrm{G}$ & (No pyrite, some etching) & \\
\hline $45-2,20-24$ & 425.80 & $\mathrm{M} / \mathrm{G}$ & (No pyrite, some etching) & \\
\hline $46-1,60-63$ & 434.40 & $\mathrm{M}$ & (Orange color, no pyrite) & \\
\hline $46-3,60-63$ & 437.40 & M & (Orange color, no pyrite) & \\
\hline $46-5,60-63$ & 440.40 & M & (Orange color, abund. pyrite) & \\
\hline $47-4,74-74$ & 448.74 & $\mathrm{M} / \mathrm{G}$ & (No pyrite, white) & \\
\hline $47-5,55-58$ & 450.05 & $\mathrm{M} / \mathrm{G}$ & (No pyrite, orange) & \\
\hline $47-6,52-55$ & 451.52 & $P$ & (Nearly barren) & \\
\hline $48-1,100-109$ & 454.20 & $\mathrm{M}$ & (Orange, no pyrite) & \\
\hline $48-3,107-110$ & 457.27 & M & (Orange, some pyrite) & M \\
\hline $48-5,104-107$ & 460.24 & M & (Lt. orange, some pyrite) & \\
\hline $49-1,118-121$ & 463.98 & $\mathrm{M} / \mathrm{G}$ & (White, no pyrite) & \\
\hline $49-5,117-120$ & 469.97 & $\mathrm{M} / \mathrm{G}$ & (Orange tinge, white, no pyrite) & \\
\hline $49-6,117$ & 471.47 & $\mathrm{M} / \mathrm{G}$ & (White, no pyrite) & \\
\hline $50-1,91-94$ & 473.41 & $\mathrm{M} / \mathrm{G}$ & (Some pyrite, orange) & \\
\hline $50-3,91-94$ & 476.41 & M & (White, no pyrite) & M \\
\hline $50-5,44-46$ & 478.94 & $\mathrm{M} / \mathrm{G}$ & (White, no pyrite) & \\
\hline $51-2$ & 493.60 & M & (Orange tinge, trace pyrite) & \\
\hline $51-4,69-71$ & 487.29 & $\mathrm{M} / \mathrm{G}$ & (Orange tinge, trace pyrite) & \\
\hline $51-5,94-97$ & 489.04 & $\mathrm{M} / \mathrm{G}$ & (Orange tinge, low abund., no pyrite) & \\
\hline $52-2,45-48$ & 493.75 & $\mathrm{M} / \mathrm{G}$ & (Orange tinge, low abund., no pyrite) & \\
\hline $52-3,45-48$ & 495.25 & $\mathrm{M}$ & (Trace pyrite, white) & \\
\hline $52-5,52-55$ & 498.32 & $\mathrm{M} / \mathrm{G}$ & (White, no pyrite) & \\
\hline $53-2,40-44$ & 503.30 & M & (Some pyrite, white) & \\
\hline $53-4,84-87$ & 505.24 & $\mathrm{M} / \mathrm{G}$ & (Orange, no pyrite) & \\
\hline $53-5,24-26$ & 507.74 & $\mathrm{M}$ & (Slight orange tinge, no pyrite) & \\
\hline $54-2,32-35$ & 512.82 & M & (Slight orange tinge, trace pyrite) & \\
\hline $54-6,23-27$ & 518.83 & $\mathrm{M} / \mathrm{G}$ & (White, no pyrite) & \\
\hline
\end{tabular}


Table 6 (continued).

\begin{tabular}{|c|c|c|c|c|}
\hline $\begin{array}{l}\text { Core, section } \\
\text { interval }(\mathrm{cm})\end{array}$ & $\begin{array}{l}\text { Depth } \\
\text { (mbsf) }\end{array}$ & $\begin{array}{c}\text { Benthic } \\
\text { preservation }\end{array}$ & Comments & Planktonics \\
\hline $\begin{array}{c}\text { 105-647A-55, CC } \\
56, C C \\
58, C C \\
59, C C \\
60, C C \\
61, C C \\
62-1,112-115 \\
62-3,60-63 \\
62-6,22-2 \\
63, C C \\
66-2, \\
66-3, \\
66, C C \\
67-1 \\
67-3 \\
67, C C \\
68-1 \\
68-3,74-77 \\
70-2\end{array}$ & $\begin{array}{l}521.10 \\
530.40 \\
549.80 \\
559.60 \\
577.20 \\
588.40 \\
599.22 \\
601.70 \\
605.82 \\
610.20 \\
638.30 \\
639.80 \\
640.50 \\
646.50 \\
649.50 \\
651.20 \\
656.20 \\
659.94 \\
686.70\end{array}$ & $\begin{array}{c}\mathrm{M} / \mathrm{G} \\
\mathrm{M} / \mathrm{P} \\
\mathrm{M} \\
\mathrm{M} \\
\mathrm{M} \\
\mathrm{M} / \mathrm{G} \\
\mathrm{M} / \mathrm{G} \\
\mathrm{M} / \mathrm{G} \\
\mathrm{M} / \mathrm{G} \\
\text { Barren } \\
\mathrm{G} \\
\mathrm{M} \\
\mathrm{M} \\
\mathrm{G} \\
\text { Barren } \\
\text { Barren } \\
\mathrm{G} \\
\mathrm{G} \\
\text { Silicified to } \\
\text { base of hole }\end{array}$ & $\begin{array}{l}\text { (White, no pyrite) } \\
\text { (Orange, pyrite) } \\
\text { (Low abund. orange, pyrite) } \\
\text { (Low abund. orange, pyrite) } \\
\text { (Orange tinge, trace pyrite) } \\
\text { (White, trace pyrite) } \\
\text { (White, no pyrite) } \\
\text { (White, no pyrite) } \\
\text { (White, abund. pyrite) } \\
\text { (White) } \\
\text { (White) } \\
\text { (Pyrite, orange) } \\
\text { (Pyrite, orange) } \\
\\
\text { (White, no pyrite) } \\
\text { (White, no pyrite) }\end{array}$ & \\
\hline
\end{tabular}

$\mathrm{G}=$ good; $\mathrm{M}=$ moderate $\mathrm{P}=$ poor.

Lith. Depth

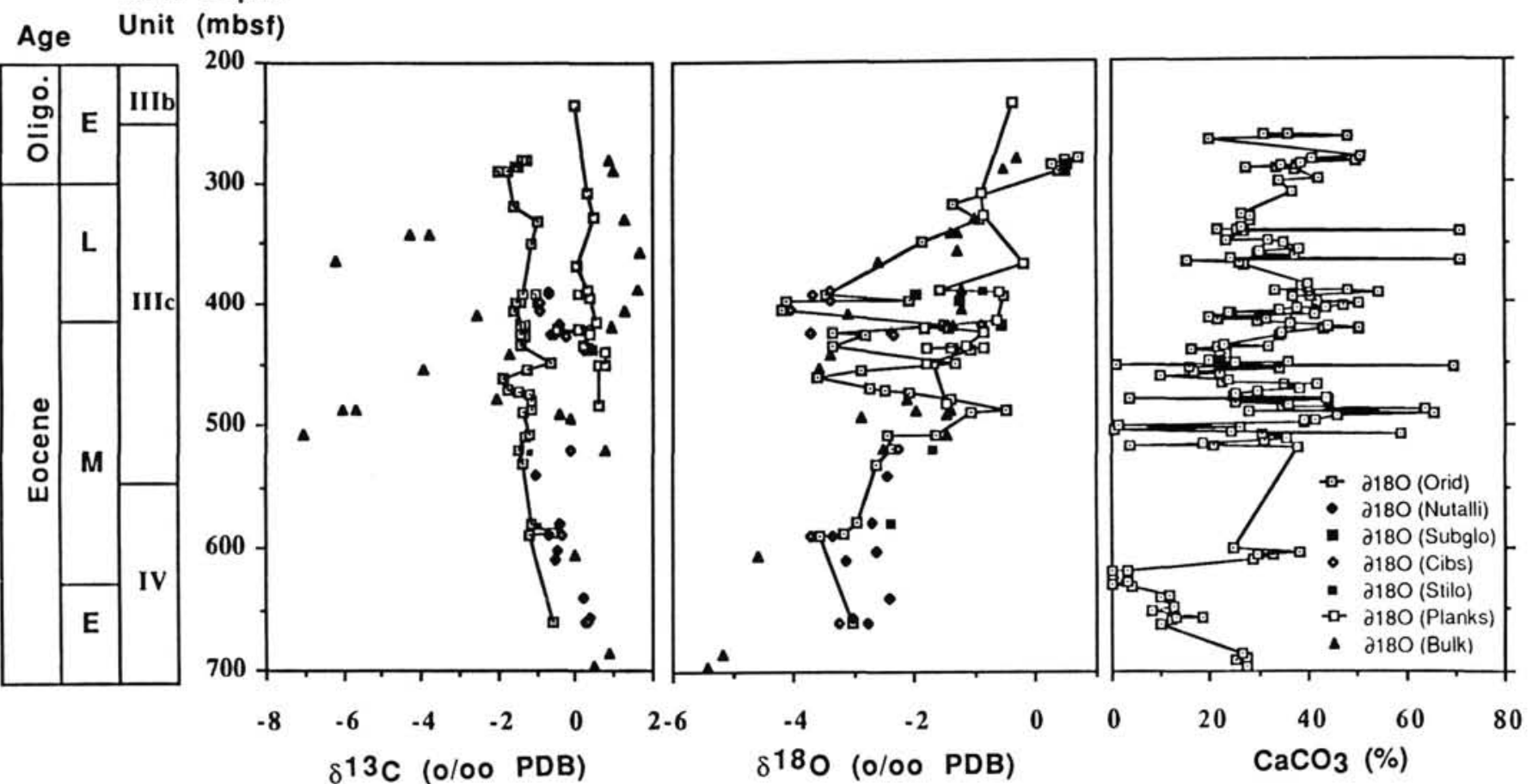

Figure 11. Stable isotope data, discrete planktonic and benthic foraminifer separates, and whole-rock samples (see key and Table 7) from Hole 647A.

cumulation rates are excessively high in the Eocene sequence, indicating that primary production may not have been particularly high. The average sedimentation rates for the Eocene and Oligocene at Site 647 , however, exceed $36 \mathrm{~m} / \mathrm{m} . y$., a relatively high rate. This may have enhanced the initial amounts of OC buried here (e.g., Müller and Suess, 1979), in comparison with other regions having similar OC rain rates but lower rates of sediment accumulation. As for the deep-water mass paleo-oxygen levels, the substantial numbers of agglutinated benthic foraminifers suggest that concentrations were low (see Kaminski et al., this volume). If so, the oxidation of $\mathrm{OC}$ before burial may have been somewhat reduced.

An alternative, or possibly complementary, explanation for the more negative carbon compositions, is that the $\delta^{13} \mathrm{C}$ values of the benthic foraminifers were altered during diagenesis. This would require that during the process of dissolution/recrystallization, a source of isotopically light dissolved carbon (derived from degradation of $\mathrm{OC}$ ) be present. The diagenetic process could have occurred soon after burial when significant quantities of $\mathrm{OC}$ were still available, but the lighter oxygen-isotope 
Table 7. Stable-isotope results from planktonic and benthic foraminifers and bulk samples.

\begin{tabular}{|c|c|c|c|c|}
\hline $\begin{array}{l}\text { Core, section, } \\
\text { interval }(\mathrm{cm})\end{array}$ & Species & $\begin{array}{l}\text { Depth } \\
\text { (m) }\end{array}$ & $\begin{array}{c}\delta^{18} \mathrm{O} \\
(\mathrm{PDB})\end{array}$ & $\begin{array}{c}\delta^{13} \mathrm{C} \\
(\mathrm{PDB})\end{array}$ \\
\hline $105-647 \mathrm{~A}-25-2,28-30$ & Unicavus & 233.80 & -0.38 & -0.01 \\
\hline $33 \mathrm{cc}$ & Mixed planks & 308.30 & -0.90 & 0.30 \\
\hline $35 \mathrm{cc}$ & Mixed planks & 327.60 & -0.84 & 0.46 \\
\hline $39-2,16-18$ & Eoceana & 367.60 & -0.21 & 0.01 \\
\hline $41-3,29-31$ & Unicavus & 388.69 & -1.58 & 0.29 \\
\hline $41-5,29-31$ & Unicavus & 391.69 & -0.59 & 0.09 \\
\hline $42 \mathrm{cc}$ & Mixed planks & 395.10 & -0.54 & 0.39 \\
\hline $44-1,79-81$ & Unicavus & 415.29 & -0.63 & 0.53 \\
\hline $44-4,115-117$ & Eoceana & 420.15 & -1.44 & -0.45 \\
\hline $44-4,115-117$ & Unicavus & 420.15 & -1.84 & 0.07 \\
\hline $45 \mathrm{cc}$ & Mixed planks & 424.10 & -0.85 & 0.37 \\
\hline $46 \mathrm{cc}$ & Mixed planks & 433.80 & -1.15 & 0.23 \\
\hline $46-2,40-42$ & Unicavis & 435.50 & -1.41 & 0.34 \\
\hline $46-2,40-42$ & Eoceana & 435.50 & -1.78 & 0.26 \\
\hline $46 \mathrm{R}, 2$ & Unicavus & 436.80 & -1.37 & 0.30 \\
\hline $46 \mathrm{R}, 2$ & Eoceana & 436.80 & -0.84 & 0.41 \\
\hline $46-3,40-42$ & Unicavus & 437.20 & -1.36 & 0.39 \\
\hline $46-4,40-42$ & Unicavus & 438.70 & -1.06 & 0.74 \\
\hline $47-5,20-22$ & Unicavus & 449.70 & -1.67 & 0.58 \\
\hline $47-5,20-22$ & Eoceana & 449.70 & -1.80 & 0.75 \\
\hline $51 \mathrm{cc}$ & Eoceana & 482.10 & -1.46 & 0.57 \\
\hline $30-1,107-109$ & Oridorsalis & 280.47 & 0.72 & -1.39 \\
\hline $30-2,25-27$ & Oridorsalis & 281.15 & 0.49 & -1.25 \\
\hline $30-4,107-109$ & Oridorsalis & 284.97 & 0.28 & -1.56 \\
\hline $30-4,107-109$ & G. Subglobosa & 284.97 & 0.52 & -1.46 \\
\hline $31-1,132-134$ & Oridorsalis & 290.32 & 0.39 & -1.76 \\
\hline $31-1,132-134$ & G. Subglobosa & 290.32 & 0.49 & -1.98 \\
\hline $33 \mathrm{cc}$ & Oridorsalis & 318.00 & -1.35 & -1.61 \\
\hline $35-3,77-79$ & Oridorsalis & 331.37 & -0.92 & -0.97 \\
\hline $36-2,49-51$ & Oridorsalis & 349.19 & -1.86 & -1.16 \\
\hline $41-3,58-61$ & Cibicidoides & 388.98 & -3.38 & -0.71 \\
\hline $41-3,58-61$ & Stilostomella & 388.98 & -0.84 & -0.62 \\
\hline $41-5,58-60$ & Oridorsalis & 391.98 & -3.47 & -1.39 \\
\hline $41-5,58-60$ & G. Subglobosa & 391.98 & -1.96 & -1.05 \\
\hline $41-5,58-61$ & Cibicidoides & 391.98 & -3.67 & -0.70 \\
\hline $42-3,32-34$ & Oridorsalis & 398.42 & -2.07 & -1.53 \\
\hline $42-3,32-34$ & G. Subglobosa & 398.42 & -1.24 & -0.96 \\
\hline $42-3,33-35$ & Cibicidoides & 398.43 & -3.38 & -0.90 \\
\hline $41-3,58-61$ & Oridorsalis & 398.98 & -4.13 & -1.45 \\
\hline $43-1,95-97$ & Oridorsalis & 405.75 & -4.19 & -1.61 \\
\hline $43-1,95-98$ & Cibicidoides & 405.75 & -4.04 & -0.94 \\
\hline $44-3,24-26$ & Oridorsalis & 417.74 & -1.50 & -1.31 \\
\hline $44-3,24-26$ & G. Subglobosa & 417.74 & -0.56 & -1.42 \\
\hline $44-3,42-46$ & Cibicidoides & 417.92 & -0.88 & -0.40 \\
\hline $45-1,14-16$ & Oridorsalis & 424.24 & -3.34 & -1.43 \\
\hline $45-1,14-16$ & Nuttallides & 424.24 & -2.36 & -0.62 \\
\hline $45-1,14-18$ & Cibicidoides & 424.24 & -3.71 & -0.52 \\
\hline $45-2,20-22$ & Oridorsalis & 425.80 & -2.81 & -1.33 \\
\hline $45-2,20-24$ & Cibicidoides & 425.80 & -2.34 & -0.22 \\
\hline $46-1,60-62$ & Oridorsalis & 434.40 & -3.36 & -1.43 \\
\hline $47-5,55-57$ & Oridorsalis & 448.65 & -1.32 & -0.63 \\
\hline $48-1,106-108$ & Oridorsalis & 454.26 & -2.88 & -1.28 \\
\hline $48-5,104-106$ & Oridorsalis & 460.24 & -3.61 & -1.89 \\
\hline $49-5,117-119$ & Oridorsalis & 469.97 & -2.72 & -1.76 \\
\hline $49-6,117-119$ & Oridorsalis & 471.47 & -2.48 & -1.51 \\
\hline $50-1,91-93$ & Oridorsalis & 473.41 & -2.10 & -1.22 \\
\hline $50-5,44-46$ & Oridorsalis & 478.94 & -1.39 & -1.12 \\
\hline $51-4,69-71$ & Oridorsalis & 487.29 & -0.48 & -1.17 \\
\hline $51-5,49-51$ & Oridorsalis & 488.59 & -1.08 & -1.36 \\
\hline $53-4,84-87$ & Oridorsalis & 506.74 & -1.66 & -1.21 \\
\hline $53-5,24-26$ & Oridorsalis & 507.64 & -2.43 & -1.34 \\
\hline $54-6,23-27$ & Oridorsalis & 518.83 & -2.36 & -1.48 \\
\hline $54-6,23-27$ & Stilostemella & 518.83 & -1.69 & -1.13 \\
\hline $54-6,24-27$ & Cibicidoides & 518.84 & -2.25 & -0.12 \\
\hline $55 \mathrm{cc}$ & Oridorsalis & 530.30 & -2.63 & -1.36 \\
\hline $56 \mathrm{cc}$ & Nuttalides & 540.00 & -2.45 & -1.06 \\
\hline $60 \mathrm{cc}$ & Oridorsalis & 578.80 & -2.95 & -1.16 \\
\hline $60 \mathrm{cc}$ & Nuttalides & 578.80 & -2.70 & -0.40 \\
\hline $60 \mathrm{cc}$ & Stilostemella & 578.80 & -2.37 & -0.93 \\
\hline $61 \mathrm{cc}$ & Oridorsalis & 586.90 & -3.17 & -0.57 \\
\hline $61 \mathrm{cc}$ & Oridorsalis & 588.40 & -3.56 & -1.22 \\
\hline $61 \mathrm{cc}$ & Nuttalides & 588.40 & -3.35 & -0.67 \\
\hline $61 \mathrm{cc}$ & Cibicidoides & 588.40 & -3.72 & -0.34 \\
\hline $62-3,60-63$ & Nuttalides & 601.70 & -2.61 & -0.49 \\
\hline $63-1,139-141$ & N. Trumpeyi & 609.19 & -3.14 & -0.55 \\
\hline $66-3,57-59$ & N. Trumpeyi & 640.37 & -2.40 & 0.18 \\
\hline $68-1,29-31$ & N. Trumpeyi & 656.49 & -3.01 & 0.35 \\
\hline
\end{tabular}

Table 7 (continued).

\begin{tabular}{clrrr}
\hline $\begin{array}{c}\text { Core, section, } \\
\text { interval }(\mathrm{cm})\end{array}$ & \multicolumn{1}{c}{ Species } & $\begin{array}{c}\text { Depth } \\
(\mathrm{m})\end{array}$ & $\begin{array}{c}\delta^{18} \mathrm{O} \\
(\mathrm{PDB})\end{array}$ & $\begin{array}{r}\delta^{13} \mathrm{C} \\
(\mathrm{PDB})\end{array}$ \\
\hline 105-647A-68-3, 74-76 & Oridorsalis & 659.94 & -3.01 & -0.60 \\
$68-3,74-76$ & N. Trumpeyi & 659.94 & -2.78 & 0.25 \\
$68-3,74-77$ & Cibicidoides & 659.94 & -3.26 & 0.29 \\
$30-1,51-53$ & Bulk & 279.91 & -0.32 & 0.87 \\
$36-4,42-44$ & Bulk & 342.12 & -1.39 & -4.32 \\
$36-4,42-44$ & Bulk & 342.12 & -1.29 & -3.78 \\
$38-6,67-69$ & Bulk & 364.57 & -2.60 & -6.23 \\
$43-4,25-27$ & Bulk & 409.55 & -3.09 & -2.54 \\
$46-5,111-113$ & Bulk & 440.91 & -3.40 & -1.69 \\
$48-1,7-9$ & Bulk & 453.27 & -3.57 & -3.95 \\
$50-4,32-34$ & Bulk & 477.32 & -2.11 & -2.06 \\
$51-4,68-70$ & Bulk & 487.28 & -1.38 & -5.68 \\
$51-4,68-70$ & Bulk & 487.28 & -1.99 & -6.03 \\
$51-6,83-83$ & Bulk & 490.43 & -1.45 & -0.43 \\
$52-2,10-12$ & Bulk & 493.40 & -2.89 & -0.16 \\
$53-4,79-81$ & Bulk & 506.45 & -1.47 & -7.04 \\
\hline
\end{tabular}

values suggest somewhat deeper burial diagenesis, as discussed below.

\section{Bulk Sample Analyses}

Stable isotope analyses were also conducted on a limited number of bulk samples. The oxygen isotopic composition of these samples shows considerable variation with depth, similar to that observed in the benthic-foraminiferal isotope record. The heaviest measured values occur in the lower Oligocene part of the sequence, where a single sample at $279.91 \mathrm{mbsf}$ yielded a $\delta 1^{8} \mathrm{O}$ value of $-0.32 \%$. The gradual depletion in bulk-sample oxygen isotope composition occurs from 300 to $400 \mathrm{msbf}$, with a trend similar to that recorded by the benthic foraminifers. Below $400 \mathrm{mbsf}$, bulk-sample $\delta^{18} \mathrm{O}$ values vary between -3.57 and $-1.38 \%$, with the lighter values generally corresponding to the lighter benthic foraminiferal values. The correspondence between bulk-sample and benthic foraminifers records suggest that burial diagenesis affected the isotopic composition of the benthics, as well as the bulk carbonate (mainly fine fraction).

A simple model can be constructed that constrains the timing and conditions under which burial diagenesis here may have occurred. Using a slightly modified equation of Killingley (1983) and assuming a closed system, a set of simple equations can be derived to calculate the "final" composition of the recrystallized carbonate $\left(\delta^{18} \mathrm{O}_{\mathrm{r}-\mathrm{f}}\right)$, as well as the overall effect on the bulk-carbonate composition $\left(\delta^{18} \mathrm{O}_{\mathrm{b}-\mathrm{f}}\right)$, if the initial composition of the carbonate is known or can be estimated.

$$
\begin{gathered}
\delta^{18} \mathrm{O}_{r-f}=M_{C} R\left[\delta^{18} \mathrm{O}_{b-i}+10^{3}(1-\alpha T)\right]+M_{i w} \delta^{18} \mathrm{O}_{i w}(1) \\
\left(M_{i w}+M_{C}\right) \alpha T . \\
\delta^{18} \mathrm{O}_{b-f}=R \delta^{18} \mathrm{O}_{r-f}+(1-R) \delta^{18} \mathrm{O}_{b-i} .
\end{gathered}
$$

In these equations, $\delta^{18} \mathrm{O}_{b-i}$ is the initial composition of the bulk carbonate and $R$ is the percentage of the bulk carbonate that undergoes dissolution and/or recrystallization. The initial composition of the interstitial waters, $\delta^{18} \mathrm{O}_{i w}$ is derived from analyses of Zachos and Cederberg (Fig. 12A and this volume), which show a $-0.88 \%$ (SMOW) $/ 100 \mathrm{~m}$ gradient at this site. The initial bottom-water oxygen isotopic composition is assumed to be $0 \%$ (SMOW). The number of moles of carbonate, $M_{C}$, and interstitial water, $\boldsymbol{M}_{i w}$ at the site of recrystallization, were determined using wet bulk density (WBD) (Srivastava, Arthur, et al., 1987) and percent carbonate $\left(\% \mathrm{CaCO}_{3}\right)$ data (Fig. 1; Table 1) in the following equations: 


$$
\begin{aligned}
& M_{C}=\left[(\mathrm{WBD}-P[1.01 \mathrm{~g}]) \% \text { (fraction) } \mathrm{CO}_{3}\right] / 100 \mathrm{~g}, \\
& M_{i w}=[P(1.01 \mathrm{~g})] / 18 \mathrm{~g} \text { (closed-system formulation), } \\
& P \quad=P_{o} \mathrm{e}^{-c z},
\end{aligned}
$$

where $P$, porosity at the time of diagenesis, was estimated using a compaction equation (5) in which $P_{o}$ is the original porosity at the time of deposition, $c$ is the compaction factor, which is a function of the major sediment components, and $z$ is the depth of burial. Model calculations were performed assuming that the sediment is $40 \%$ carbonate by weight. The fractionation factor $\alpha$, for calcite-water at a given temperature, was obtained from Friedman and O'Neil (1977) and $T$, the ambient temperature of the system at the time of diagenesis, was determined using the thermal gradient for this region of $7.4^{\circ} \mathrm{C} / 100 \mathrm{~m}$ (Srivastava, Arthur, et al., 1987). The initial temperature was assumed to be near that of bottom waters $\left(<10^{\circ} \mathrm{C}\right.$ for late Eocene to present).

The model was run for varying degrees of recrystallization (Fig. 12B) using two different initial $\delta^{18} \mathrm{O}$ compositions for the bulk carbonate. The first model was run assuming a modern $\delta^{18} \mathrm{O}$ composition for bulk carbonate $(+0.5$ in Fig. 12B), with an initial temperature of $5^{\circ} \mathrm{C}$ for bottom water. The second run was conducted assuming (1) that the bulk-carbonate composition is equal to that of nannofossil-carbonate, which makes up more than $95 \%$ of the carbonate, and (2) that the isotopic composition of the nannofossils is equal to that of carbonate secreted in isotopic equilibrium at ambient surface-water temperatures at the time of formation in the Eocene (-1.0 in Fig. 12B). There are few available temperature estimates for Eocene surface waters in this region, but we assumed a temperature of $16^{\circ} \mathrm{C}$, based on temperature estimates for surface waters in other early to late Eocene regions of the North Atlantic (Miller et al., 1987).

This model predicts the expected range of isotopic compositions that could be obtained theoretically for variable amounts of recrystallization and a range in burial depths. If we compare the model curves with data from Site 647, we discover that less than $50 \%$ of the original carbonate in bulk samples at $400 \mathrm{mbsf}$ needed to recrystallize in equilibrium with interstitial waters to produce the observed $\delta^{18} \mathrm{O}$ composition of $-4.0 \%$. The same oxygen isotopic composition at shallower buried depths would require a greater percentage of the carbonate to have undergone recrystallization. If we assume that most of the diagenesis occurred between 300 and 400 mbsf, from $50 \%$ to $75 \%$ of the original carbonate must have dissolved and reprecipitated as cement to obtain $\delta^{18} \mathrm{O}$ values of $-4.0 \%$.

This approach assumes that most of the diagenesis occurred at some threshold depth at which carbonate instability was reached instantaneously. Although this probably is unrealistic, the model does help to constrain the conditions under which diagenesis occurred.

Whereas the $\delta^{18} \mathrm{O}$ trends for bulk samples are easily explained in terms of burial diagenesis, the $\delta^{13} \mathrm{C}$ record requires a more complicated interpretation. Most of the samples have $\delta^{13} \mathrm{C}$ values of -2.0 to $1.0 \%$, but extremely light values in the range of -7.0 to $-6.0 \%$ were recorded for a number of bulk samples from discrete intervals. The more negative $\delta^{13} \mathrm{C}$ values occur in intervals where high concentrations of siderite and rhodochrosite were observed. Both minerals tend to precipitate at the boundaries of reducing zones, where an ample supply of $\mathrm{Fe}$, $\mathrm{Mn}$, and carbonate ions are available. The carbon isotopic composition of pore-water total dissolved carbonate in OC-rich sediments is more negative than that in average seawater, mainly due to the addition of isotopically light $\mathrm{CO}_{2}$ derived from decaying organic matter. As a result, authigenic carbonate minerals formed during early diagenesis would be isotopically light,
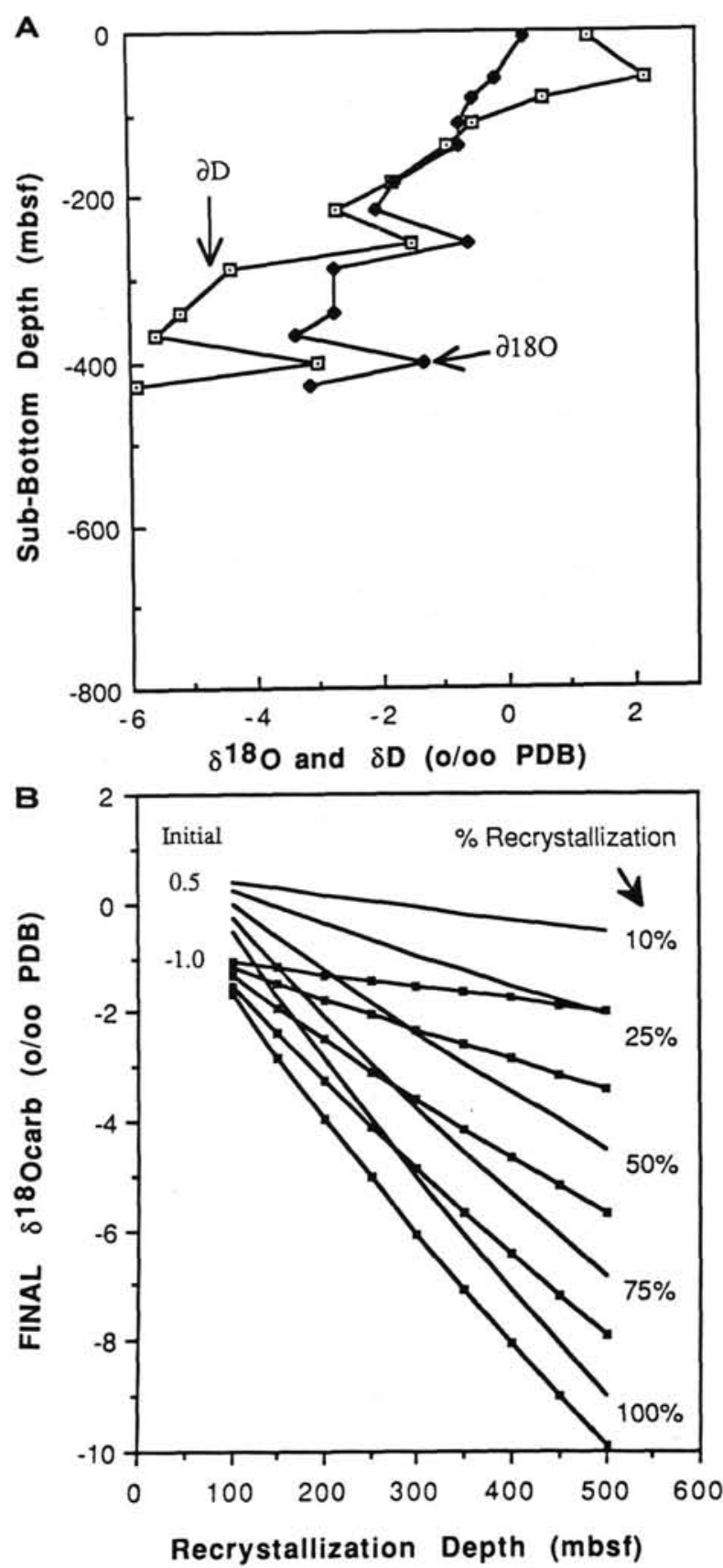

Figure 12. A. Trends in oxygen and deuterium isotopic composition of interstitial waters with depth in Hole 647A (from Zachos and Cederberg, this volume) showing downhole decrease. B. Results of a diagenetic model (see text for details) that predicts the $\delta^{18} \mathrm{O}$ of calcite recrystallized at a given depth with a geothermal gradient of $7.4^{\circ} \mathrm{C} / 100 \mathrm{~m}$. The two families of curves represent alteration of two different starting compositions for bulk carbonate $(+0.5 \%$ and $-1.0 \%$, PDB, respectively) and different proportions of calcite recrystallized at a given subbottom depth.

compared to biogenic carbonate. This conclusion is discussed later in the context of the depositional regime that prevailed at Site 647 during the Eocene/Oligocene.

\section{DISCUSSION}

There are several anomalous aspects to the geochemistry of the hemipelagic sequence at Site 647 . In particular, we noted in- 
tervals of high $\mathrm{Fe}, \mathrm{Mn}, \mathrm{P}_{2} \mathrm{O}_{5}$, and associated elements, which are related to authigenic carbonate and apatite precipitates. The origin of these and other geochemical characteristics of the Eocene-Oligocene sequence is discussed below.

The diagenetic precipitation of $\mathrm{Fe}$ and Mn-carbonate minerals in Tertiary hemipelagic sediments is not that unusual. Disseminated and nodular siderite has been described from the late $\mathrm{Pa}$ leogene and Neogene from a number of DSDP sites in the western North Atlantic (Sites 102, 104-Lancelot et al., 1972; Site 533-Matsumoto, 1983; Site 603-von Rad and Botz, 1986) and Pacific (Middle America Trench: Wada et al., 1982; Japan Trench: Matsumoto and Matsuhisa, 1986). These occurrences are in hemipelagic sequences characterized by high rates of deposition and preservation of sufficient organic matter to promote total consumption of dissolved sulfate by bacterial sulfate reduction, followed by strong methanogenesis (e.g., von Rad and Botz, 1986; Matsumoto, 1983; Claypool and Kaplan, 1984). Dolomite occurs in many of the same sediments that contain siderite. Matsumoto (1983) argued that dolomite precipitation occurs at relatively shallow depths in the Blake-Bahama Outer Ridge (Site 533) during late stages of sulfate reduction and early methanogenesis, followed by authigenic siderite at greater depth within the sediment. Similar results were obtained for Japan Trench sediments using oxygen and carbon isotope constraints (e.g., Irwin et al., 1977; Okada, 1980; Wada and Okada, 1983; Matsumoto and Matsuhisa, 1986).

The growth of $\mathrm{Fe}$ and $\mathrm{Mn}$ authigenic phases clearly requires the elevated alkalinity, $\mathrm{CO}_{3}^{2-}$ and $\mathrm{TCO}_{2}$ concentrations that accompany extensive degradation of organic matter, as well as a source of soluble $\mathrm{Fe}$ and $\mathrm{Mn}$ that are released under low redox conditions. Siderite precipitation also implies available Fe in excess of that necessary to form pyrite from the dissolved sulfide that results from bacterial sulfate reduction.

\section{Timing and Geochemical Conditions of Precipitation}

The carbon and oxygen isotopic compositions of discrete carbonate nodules provide constraints on the timing of precipitation and origin of the authigenic Fe-Mn carbonates (e.g., Irwin et al., 1977; Gautier, 1985). Carbon isotopic compositions of the homogenized nodules (no attempt was made to examine possible zonation of nodules in this study) range between -2 and $-6 \%$ (PDB). These values indicate that carbonate ion was drawn from a somewhat ${ }^{13} \mathrm{C}$-depleted pore-water carbon reservoir that resulted from oxidation of organic matter. Pore-water dissolved sulfate concentrations (Zachos and Cederberg, this volume) reach a minimum at 500 mbsf but do not decrease below about $13 \mathrm{mmol} / \mathrm{L}$. Thus, sufficient reactive organic matter may have been available to promote consumption of initial dissolved oxygen and partial bacterial sulfate reduction, but methanogenesis may never have occurred. This was confirmed when monitoring of head-space gas during drilling at Site 647 detected no methane (Srivastava, Arthur, et al., 1987). Present sedimentary organic carbon contents are generally low, ranging from 0.2 to $0.5 \mathrm{wt} \%$ (Fig. 1). Because there was insufficient pore water with which to determine total dissolved inorganic carbon (TDC) or $\delta^{13} \mathrm{C}_{\mathrm{TDC}}$, we must estimate the $\delta^{13} \mathrm{C}_{\mathrm{TDC}}$ on the basis of a $\Delta \mathrm{SO}_{4}^{2-}$ of about $15 \mathrm{mmol} / \mathrm{L}$, assuming a $\Delta \mathrm{SO}_{4}^{2-}$ : $\mathrm{HCO}_{3}^{-}$ratio of $1: 2$ (TDC increases in proportion to the sulfate decrease) during sulfate reduction and $\mathrm{a}-22 \% \delta^{13} \mathrm{C}$ value for the largely marine organic matter undergoing oxidation. The calculated minimum pore-water $\delta^{13} C_{T D C}$ value is about $-20 \%$. Therefore, it is likely that the authigenic Fe-Mn carbonates precipitated during early diagenesis, before the main phase of bacterial sulfate reduction, or that the Fe-Mn carbonates are a partial replacement of preexisting calcium carbonate having initially heavier $\delta^{13} \mathrm{C}$. The $\delta^{18} \mathrm{O}$ values of the nodular carbonates have a range of -1.30 to $-3.50 \%$ (PBD) (Table 7). Using these data to estimate temperatures of formation is difficult because oxygen-isotopic fractionation factors between water and siderite and phosphoric acid and siderite have only recently been experimentally determined (Carothers et al., 1988). The isotope fractionation effects of bonding of different metals with carbonate also have been estimated theoretically (Tarutani et al., 1969), and Sharma and Clayton (1965) reported the fractionation factor for rhodochrosites. Siderite should be about $0.9 \%$ to $1.5 \%$ heavier than coexisting calcite; whereas, rhodochrosite should be similar in composition to coprecipitated calcite. Using an assumed $\delta^{18} \mathrm{O}$ of Eocene seawater of $-1.2 \%$ (SMOW; pre-glacial), the estimated temperature range for siderite or rhodochrosite precipitation would be $28^{\circ}$ to $30^{\circ} \mathrm{C}$. Estimated bottom-water temperatures for the Atlantic Ocean during the mid-to-late Eocene range from $8^{\circ}$ to $12^{\circ} \mathrm{C}$ (e.g., Shackleton, 1986; Miller et al., 1985), so that the $\delta^{18} \mathrm{O}$ data from the nodules suggest precipitation following some burial. Pore water $\delta^{18} \mathrm{O}$ at Site 647 (below about $300 \mathrm{mbsf}$ ) is presently about $-3 \%$ (SMOW; Zachos and Cederberg, this volume), which at in-situ temperatures of about $35^{\circ} \mathrm{C}$, would produce siderite with a $\delta^{18} \mathrm{O}$ of about -5 to $-6 \%$ (PDB). Therefore, the authigenic carbonate nodules formed at shallow depths of burial, perhaps no more than 100 to $200 \mathrm{~m}$. Separation of pure mineral phases for isotopic studies was not possible, and one must keep in mind that we analyzed only mixtures, which precludes a more detailed interpretation of the $\delta^{18} \mathrm{O}$ values at this time.

Whereas previous research suggests an association of $\mathrm{Fe}$ - and Mn-carbonates with methanogenesis, our data from Site 647 indicate that such geochemical conditions are not a prerequisite (see also Suess, 1979). However, there is little doubt that such mineralization is more extensive in methane-associated settings, which may be true for anoxic dolomites as well (e.g., Kelts and McKenzie, 1982).

One can see by inspection that the highest $\mathrm{Fe} / \mathrm{Al}$ ratios (Fig. 13) occur in the intervals of documented increase in the relative abundance of concretions or nodules and that some, but not all, of the high $\mathrm{Fe} / \mathrm{Al}$ ratios correspond to intervals of higher $\mathrm{Fe} / \mathrm{Mn}$ as well. We would expect redox separation of Fe from $\mathrm{Mn}$, as it has been described from many modern environments (e.g., Froelich et al., 1979; Li, 1982). Because of the effect of dilution of $\mathrm{Al}$ by higher carbonate contents and because both $\mathrm{Fe}$ and $\mathrm{Mn}$ are incorporated in the carbonate phases in varying amounts, we have also plotted the moving correlation coefficient for the relationship between $\mathrm{Fe} / \mathrm{Al}$ and $\mathrm{Fe} / \mathrm{Mn}$ ratios in Figure 13. The correlation coefficients indicate variation mainly between intervals of strong negative $(r=-0.65)$ and relatively, strong positive $(r=0.60)$ correlation. Over much of the sequence (260-415 mbsf; 465-485 mbsf) variations in Fe and Mn concentrations may be positively correlated, even though the $\mathrm{Fe} / \mathrm{Mn}$ ratio varies considerably. A few intervals of generally positive $\mathrm{Fe} / \mathrm{Al}-\mathrm{Fe} / \mathrm{Mn}$ correlation $(415-465 \mathrm{mbsf}$ and $>500$ mbsf) are mainly characterized by lower concentrations of $\mathrm{Fe}$ and Mn overall. These are most likely the "source" intervals from which the necessary "excess" Fe, and particularly excess $\mathrm{Mn}$, migrated for precipitation as authigenic phases. Coarsegrained, initially more carbonate-rich beds may have catalyzed precipitation. We were unable to confirm this by examining the cores, but the need for some sort of coarser-grained nucleation site was postulated by Pederson and Price (1982) for more recent nonmethane-associated manganese-carbonates of the Panama Basin. Bohrmann and Thiede (this volume) examined FeMn carbonates from deeper in the sequence in Hole 647A (Cores $105-647$ A- 62 through -71) and found that they commonly occur as precompaction burrow-filling cements and that $\mathrm{Mn}$ is more important than $\mathrm{Fe}$ in cation substitution into the authigenic carbonate phases. Iron concentrations are higher on average in the lower part of lithologic Unit III that we analyzed, and in nodu- 

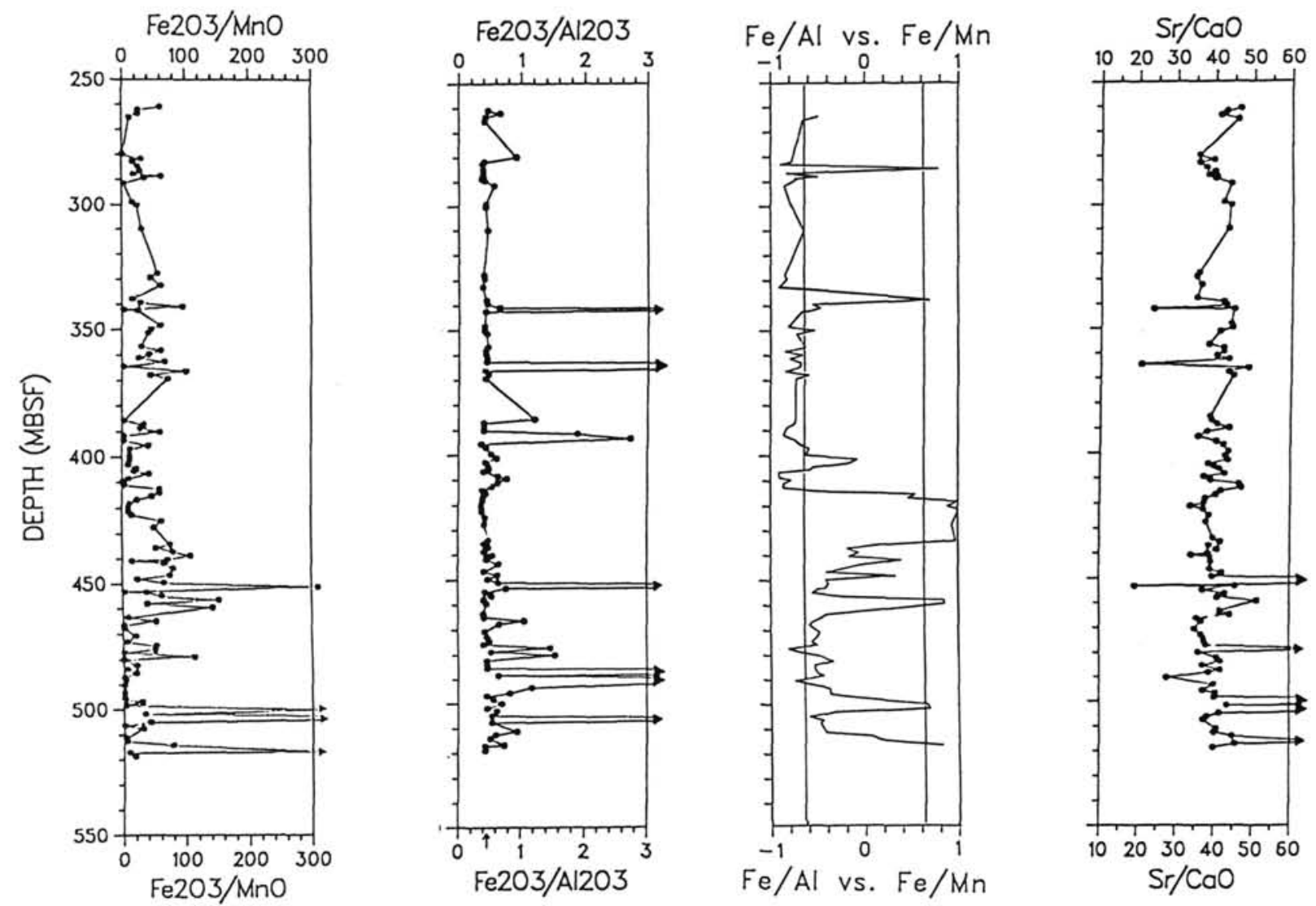

Figure 13. $\mathrm{Fe}_{2} \mathrm{O}_{3} / \mathrm{MnO}, \mathrm{Fe}_{2} \mathrm{O}_{3} / \mathrm{Al}_{2} \mathrm{O}_{3}$, moving correlation coefficients of $\mathrm{Fe} / \mathrm{Al}$ vs. $\mathrm{Fe} / \mathrm{Mn}$ and $\mathrm{Sr} / \mathrm{CaO}\left(\times 10^{-4}\right)$ for lithologic Unit IIIC, Hole $647 \mathrm{~A}$.

lar or concretionary horizons in general, than they are in the Unit IV samples analyzed by Bohrmann and Thiede (this volume).

Although those authors hypothesized that possible hydrothermal oxyhydroxides were a source of excess $\mathrm{Mn}$ and $\mathrm{Fe}$ for lithologic Unit IV, our geochemical data for Unit III do not require a significant hydrothermal flux. The average or baseline $\mathrm{Fe} / \mathrm{Al}$ ratio (Fig. 13-see arrow) is similar to or only slightly $\mathrm{Fe}$ enriched, compared to that in abyssal red clay of the recent North Atlantic (V26-157; Table 3). However, the average Mn concentration in "background" samples at Site 647 (Table 3) is higher by a factor of four on a carbonate-free basis and the $\mathrm{Mn} / \mathrm{Al}$ ratio higher by a factor of five. This might suggest some additional hydrothermal flux to the site, and other indications of this might appear in the geochemical data. For example, Figure 14 is a comparison of the geochemistry (carbonate-free) of 99 background (nonconcretionary, good correspondence between $\mathrm{CC}$ as $\mathrm{CaCO}_{3}$ and $\mathrm{Ca}$ as $\mathrm{CaCO}_{3}$ ) Site 647 samples with that of modern northern Atlantic abyssal red clay. This diagram illustrates that $\mathrm{Mn}, \mathrm{Co}, \mathrm{Ca}$, and $\mathrm{Ba}$ are somewhat enriched at Site 647 , which could also be related to a minor hydrothermal detritus flux (see below). Phosphate is also enriched in some of the concretionary horizons (Figs. 3 and 15) and occurs as a discrete authigenic apatite phase. The origin of the $\mathrm{P}$ enrichments is probably related to desorption of $\mathrm{P}$ from $\mathrm{Fe}-\mathrm{Mn}$ oxyhydroxides during chemical reduction and dissolution of those phases (e.g., Froelich et al., 1979). Sr is relatively enriched in carbonatephosphatic nodules (Figs. 13 and 15), but not in the Fe- and $\mathrm{Mn}$-rich carbonates. Because the $\mathrm{Sr} / \mathrm{Ca}$ ratio in pore waters increases with depth (Zachos and Cederberg, this volume), the in-

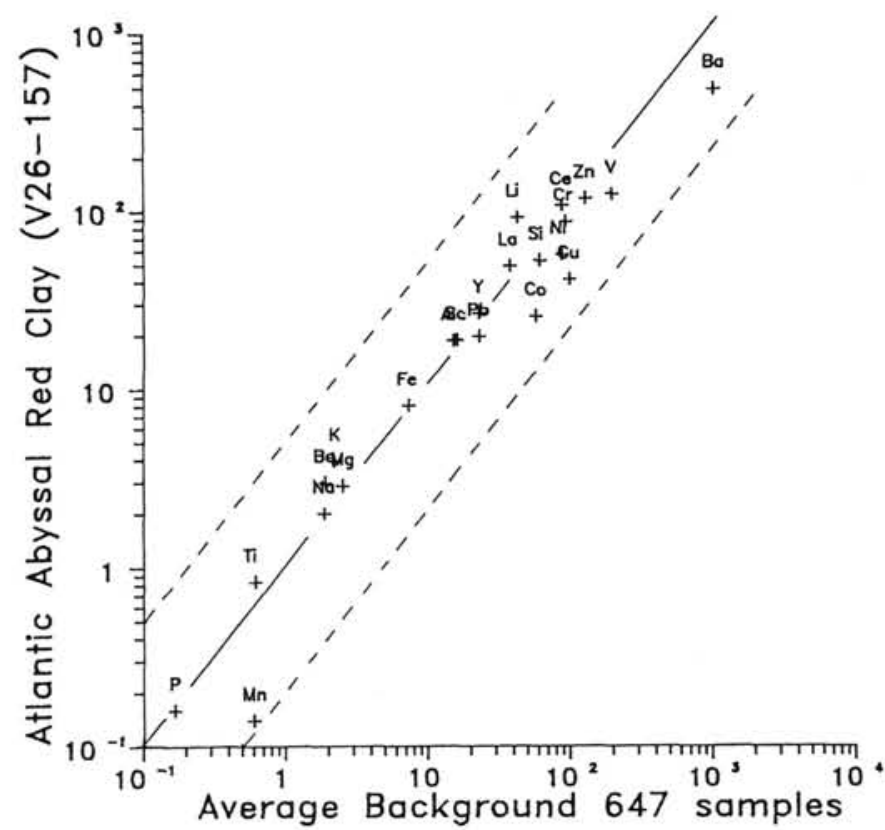

Figure 14. Plot comparing elemental concentrations of average "background" Site 647 samples $(n=99)$ on carbonate-free basis with those for Atlantic abyssal red clay (V26-157). Solid line represents a 1:1 correspondence. 

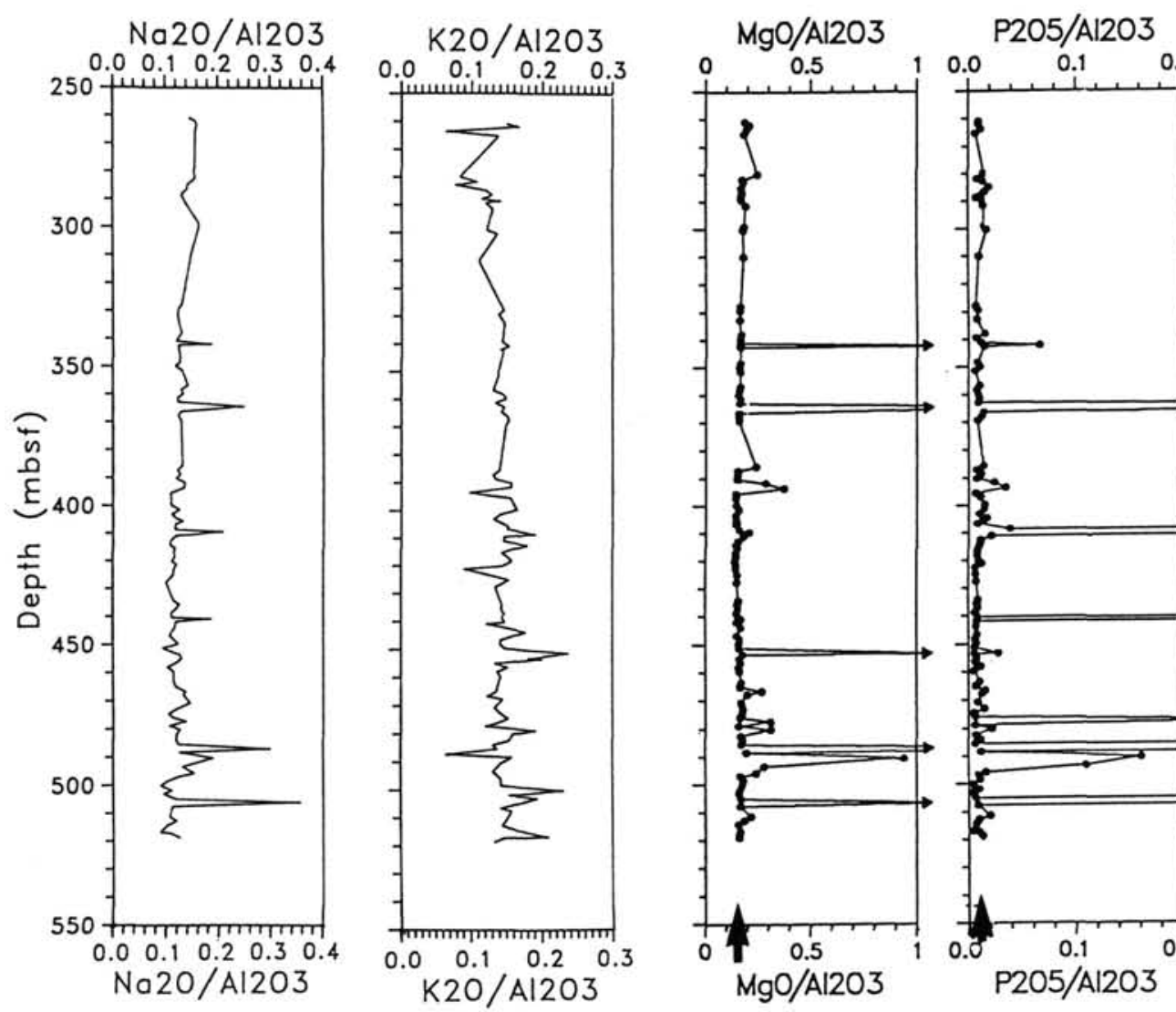

Li/A1203
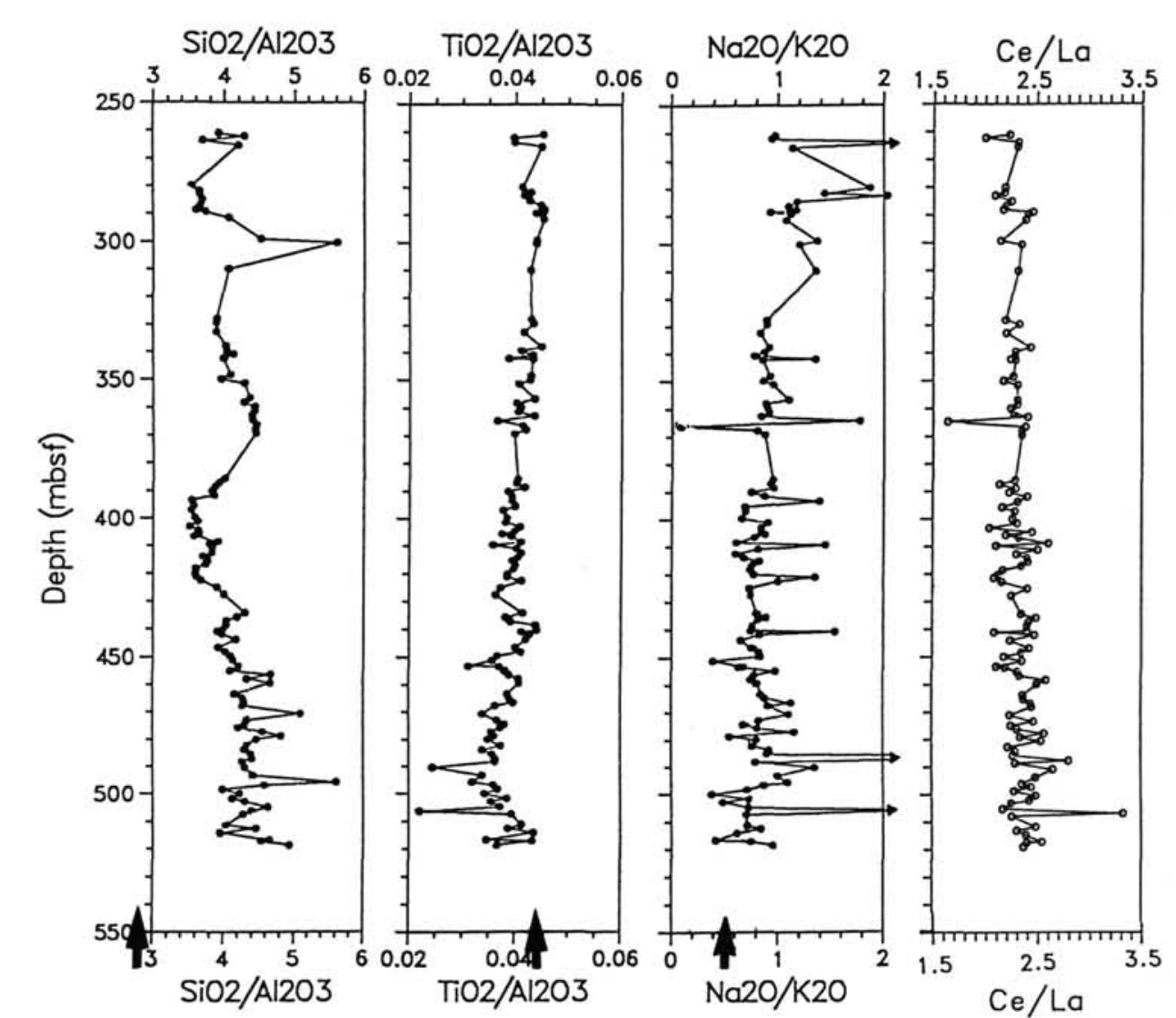

Figure 15. A. $\mathrm{SiO}_{2} / \mathrm{Al}_{2} \mathrm{O}_{3}, \mathrm{TiO}_{2} / \mathrm{Al}_{2} \mathrm{O}_{3}, \mathrm{Na}_{2} \mathrm{O} / \mathrm{K}_{2} \mathrm{O}$, and $\mathrm{Ce} / \mathrm{La}$ ratios plotted downhole for lithologic Unit IIIC of Hole 647; arrows on bottom axis denote average Atlantic red clay (V26-157) values. $\mathrm{B}$. $\mathrm{Na}_{2} \mathrm{O} / \mathrm{Al}_{2} \mathrm{O}_{3}, \mathrm{~K}_{2} \mathrm{O} / \mathrm{Al}_{2} \mathrm{O}_{3}, \mathrm{MgO}_{\mathrm{Al}} \mathrm{Al}_{3}, \mathrm{P}_{2} \mathrm{O}_{5} / \mathrm{Al}_{2} \mathrm{O}_{3}$, and $\mathrm{Li}$ / $\mathrm{Al}_{2} \mathrm{O}_{3}$ values for lithologic Unit IIIC of Hole 647A plotted downcore. 
creased $\mathrm{Sr} / \mathrm{Ca}$ ratio in some nodules may indicate later-stage precipitation.

\section{Other Geochemical Signals}

Major-element aluminum ratios (Fig. 15) overall exhibit little change from the base to the top of the sequence. However, there are several important trends to note. The $\mathrm{Si} / \mathrm{Al}$ ratio varies from above 4 in the middle Eocene (430-525 mbsf) to a low of about 3.5 at the middle/upper Eocene boundary and fluctuates somewhat above that level to a high centered at about $310 \mathrm{mbsf}$. For this study our samples were collected from well below the lower Oligocene biogenic opal-rich sequence (above $240 \mathrm{mbsf}$ ). However, it is likely that the $\mathrm{Si} / \mathrm{Al}$ variations reflect changes in the original amounts of biogenic silica in the Eocene, suggesting that the mid-middle Eocene and latest Eocene were times of higher surface-water opal production. Our data confirm and amplify the conclusions of Bohrmann and Stein (this volume) and Nielsen et al. (this volume), who used visual and geochemical and X-ray mineralogical techniques, respectively. On the basis of these studies, most of the original opal may have dissolved and reprecipitated as authigenic smectite or opal CT. The $\mathrm{Mg}$ / $\mathrm{Al}$ ratio concentration is also relatively constant (Fig. 15), with the exception of high $\mathrm{Mg}$ spikes that are associated with $\mathrm{Fe}-\mathrm{Mn}$ carbonate horizons. $\mathrm{Mg}$ probably was substituted in the lattice of these carbonates. However, no discrete dolomite was detected in our X-ray mineralogical studies.

$\mathrm{Ce} / \mathrm{La}$ and $\mathrm{Li} / \mathrm{Al}$ ratios decrease gradually upsection (Fig. 15). This is interesting in light of the possible decreasing importance of a minor hydrothermal component. However, Li concentrations in pore waters also increase downhole (Zachos and Cederberg, this volume), so that the prevalence of authigenic smectite formation in the lower part of Unit III and in Unit IV (Bohrmann and Stein; Nielsen et al., this volume) might explain the higher sediment $\mathrm{Li} / \mathrm{Al}$ ratios. Concentrations of $\mathrm{Li}$ and REEs, as well as those of $\mathrm{Mn}, \mathrm{V}, \mathrm{Zn}, \mathrm{Ni}$, and $\mathrm{Cu}$ are relatively enriched in comparison to Atlantic red clays (Fig. 14). Higher $\mathrm{Ba}$ and $\mathrm{Co}$ concentrations would result from somewhat higher productivity and organic-associated fluxes in the Eocene at Site 647 in comparison to the central North Atlantic Ocean today. Biogenic detritus, particularly marine particulate organic matter, incorporates or absorbs trace elements (e.g., Bostrøm et al., 1974; Collier and Edmond, 1974) as do fine-grained clay minerals (e.g., Balistrieri et al., 1981). At this point, it is difficult to separate the potential variations in sediment adsorption capacity in this clay-rich sediment (e.g., Balistrieri and Murray, 1984) from potential hydrothermal sources.

Finally, $\mathrm{Ti} / \mathrm{Al}, \mathrm{Na} / \mathrm{Al}$, and $\mathrm{Na} / \mathrm{K}$ ratios increase significantly above 320 mbsf (uppermost Eocene). This may reflect an increase in average sediment grain-size (Nielsen et al., this volume) and increasing feldspar and detrital clay proportions as opposed to smectite, related to climate deterioration and an increase in bottom-current strength near the Eocene/Oligocene boundary.

\section{CONCLUSIONS}

The geochemistry of background sediment of lithologic Unit III at 647 of middle Eocene to lower Oligocene age is remarkably constant and similar to that of modern Atlantic red clay, which has a distinctly terrigenous geochemical composition. Nonetheless, the high sedimentation rates (average $36 \mathrm{~m} / \mathrm{m} . \mathrm{y}$.) over this interval (260-515 mbsf) led to some initial preservation of organic matter and to anoxic early diagenesis and sulfate reduction. Sulfate reduction led to reduction of Fe and Mn oxyhydroxides, to increased pore-water alkalinity and $\mathrm{TCO}_{2}$ and to higher pore-water phosphate concentrations, all of which allowed precipitation of authigenic Fe-Mn carbonates and apatite at a number of horizons in the sequence.
Because of this early diagenesis, there may be no opportunity to unravel paleoenvironment changes from the stable isotopic composition of biogenic carbonate phases. In particular, although benthic foraminiferal preservation visually is moderate-to-good over most of the sequence, both oxygen and carbon isotopic compositions indicate significant burial diagenesis. Such pronounced alteration was unexpected in such shallowly buried, relatively clay-rich and organic carbon-poor sediments.

The geochemical data suggest that there was a possible minor hydrothermal component to the sediment, the proportion of which wanes through time and distance from the potential source. Concentrations of $\mathrm{Mn}, \mathrm{Co}, \mathrm{Cu}$, and $\mathrm{Ba}$ are higher than expected for a purely terrigenous source, and the relatively high $\mathrm{Li} / \mathrm{Al}$ and $\mathrm{Ce} / \mathrm{La}$ ratios may indicate a hydrothermal component as well. $\mathrm{Si} / \mathrm{Al}$ ratios are high in upper middle Eocene and upper Eocene portions of the sequence, confirming biogenic opal flux to the seafloor at those times, which are inferred to have been characterized by higher paleoproductivity. Average $\mathrm{Na} / \mathrm{K}$ ratios are constant through the sequence, except near the top of Unit IIIC, where the ratio increases significantly. This occurs in conjunction with an increase in the relative proportion of silt-sized sediment and in feldspar concentrations, which suggest that the feldspar is a more sodium-rich variety.

Future more-detailed geochemical studies and comparison of units above and below Unit IIIC will help elucidate the question of the importance of a hydrothermal source for some sedimentary components.

\section{ACKNOWLEDGMENTS}

We would like to acknowledge the help of the crew of the JOIDES Resolution, without whose efforts these samples would not have been available. We appreciate the efforts of Rhonda Kenny in preparation of this manuscript. Part of this study was funded by JOI-USSAC Contract 76322 to MAA.

\section{REFERENCES}

Addy, S. K., 1979. Rare earth element patterns in manganese nodues and micronodules from northwest Atlantic. Geochim. Cosmochim. Acta, 43:1105-1115.

Arthur, M. A., Hagerty, S., Dean, W. E., Claypool, G. E., Daws, T. A., McManaman, D., Meyers, P. A., and Dunham, K., 1987. A geochemical note: comparison of techniques for obtaining $\mathrm{CaCO}_{3}$, organic carbon, and total nitrogen in limestones and shales. In van Hinte, J. E., Wise, S. W., Jr., et al., Init. Repts. DSDP, 92: Washington (U.S. Govt. Printing Office), 1263-1268.

Baedecker, P. (Ed.), 1987. Methods for Geochemical Analysis: Washington (U.S. Govt. Printing Office), USGS Bull. 1770.

Balistrieri, L., Brewer, P. G., and Murray, J. W., 1981. Scavenging residence times of trace metals and surface chemistry of sinking particles in the deep ocean. Deep-Sea Res., 28(A):101-121.

Balistrieri, L. S., and Murray, J. W., 1984. Marine scavenging: trace metal adsorption by interfacial sediment from MANOP Site $\mathrm{H}$. Geochim. Cosmochim. Acta, 48:921-929.

Bischoff, J. L., Heath, G. R., and Leinen, M., 1979. Geochemistry of deep-sea sediments from the Pacific manganese nodule province: DOMES Sites A, B, and C. In Bischoff, J. L., and Piper, D. Z. (Eds.), Marine Geology and Oceanography of the Pacific Manganese Nodule Province: New York (Plenum Press), Mar. Sci. Ser., 9: $397-436$.

Boersma, A., Premoli-Silva, I., and Shackleton, N. J., 1987. Atlantic Eocene planktonic foraminiferal paleohydrographic indicators and stable isotope paleoceanography. Paleoceanography, 2:287-331.

Bostrøm, K., Joensuu, O., and Broihm, I., 1974. Plankton: its composition and its significance as a source of pelagic sediments. Chem. Geol., 14:255-271.

Bostrøm, K., and Peterson, M.N.A., 1969. The origin of aluminumpoor ferromanganoan sediments in areas of high heat flow on the East Pacific Rise. Mar. Geol., 7:427-447.

Carothers, W. W., Adami, L. H., and Rosenbauer, R. J., 1988. Experimental oxygen-isotope fractionation between siderite-water and phosphoric acid liberated $\mathrm{CO}_{2}$-siderite. Geochim. Cosmochim. Acta, 52: 2445-2450. 
Chester, R., and Aston, S. R., 1976. The geochemistry of deep-sea sediments. In Riley, J. P., and Chester, R. (Eds.), Chemical Oceanography: New York (Academic Press), 6:281-390.

Claypool, G. E., and Kaplan, I. R., 1974. The origin and distribution of methane in marine sediments. In Kaplan, I. R. (Ed.), Natural Gases in Marine Sediments: New York (Plenum Press), 99-139.

Collier, R., and Edmond, J., 1984. The trace element geochemistry of marine biogenic particulate matter. Prog. Oceanogr., 13:113-199.

Dean, W. E., and Pahrduhn, N. L., 1984. Inorganic geochemistry of sediments and rocks recovered from the southern Angola Basin and adjacent Walvis Ridge, Sites 530 and 532, Deep Sea Drilling Project Leg 75. In Hay, W. W., Sibuet, J.-C., et al., Init. Repts. DSDP, 75 (Pt. 2): Washington (U.S. Govt. Printing Office), 923-958.

Friedman, I., and O'Neil, J. R., 1977. Compilation of stable isotope fractionation factors of geochemical interest. In Fleischer, M. (Ed.), Data of Geochemistry. U.S. Geol. Surv. Prof. Pap., 440KK.

Froelich, P. N., Klinkhammer, G, P., Bender, M. L., Luedtke, N. A., Heath, G. R., Cullen, D., Dauphin, P., Hammond, D., Hartman, B., and Maynard, V., 1979. Early oxidation of organic matter in pelagic sediments of the eastern equatorial Atlantic: suboxic diagenesis. Geochim. Cosmochim. Acta, 43:1075-1090.

Goldschmidt, V. M., 1958. Geochemistry: London (Oxford Univ. Press).

Huffman, E.W.D., 1977. Performance of a new automatic carbon dioxide coulometer. Microchem. J., 22:567-573.

Irwin, H., Curtis, C. D., and Coleman, M., 1977. Isotopic evidence for source of diagenetic carbonates formed during burial of organicrich sediments. Nature, 269:209-213.

Keigwin, L., 1980. Paleoceanographic change in the Pacific at the Eocene/Oligocene boundary. Nature, 277:722-725.

Keigwin, L., and Corliss, B., 1986. Stable isotopes in Eocene/Oligocene foraminifera. Geol. Soc. Am. Bull., 163:197-236.

Kelts, K., and McKenzie, J. A., 1982. Diagenetic dolomite formation in Quaternary anoxic diatomaceous muds of Deep Sea Drilling Project Leg 64, Gulf of California. In Curray, J., Moore, D. G., et al., Init. Repts. DSDP, 64 (Pt. 2): Washington (U.S. Govt. Printing Office), 553-570.

Killingly, J., 1983. Effects of diagenetic recrystallization on ${ }^{18} \mathrm{O} /{ }^{16} \mathrm{O}$ values of deep-sea sediments. Nature, 301:594-597.

Klovan, J. E., and Miesch, A. T., 1976. Extended CABFAC QMODEL computer programs for Q-mode factor analysis of compositional data. Comp. Geosci., 1:161-178.

Lancelot, Y., Hathaway, J., and Hollister, C. D., 1972. Lithology of sediments from the western North Atlantic, Leg 11, Deep Sea Drilling Project. In Hollister, C. D., Ewing, J., et al., Init. Repts. DSDP, 11: Washington (U.S. Govt. Printing Office), 901-949.

Li, Y.-H., 1982. Interelement relationship in abyssal Pacific ferromanganese nodules and associated pelagic sediments. Geochim. Cosmochim. Acta., 46:1053-1060.

McCorkle, D. C., 1987. Stable carbon isotopes in deep-sea pore waters: modern geochemistry and paleoceanographic implications [Ph.D. dissert.]. Univ. of Washington, Seattle.

Miller, K. G., Curry, W. B., Ostermann, D. R., 1985. Late Paleogene (Eocene to Oligocene) benthic foraminiferal oceanography of the Goban Spur region. In de Graciansky, P. C., Poag, C. W., et al., Init. Repts. DSDP, 80: Washington (U.S. Govt,. Printing Office), 1089-1106.

Miller, K. G., Fairbanks, R. G., and Mountain, G. S., 1987. Tertiary oxygen isotope synthesis, sea-level history, and continental margin erosion. Paleoceanography, 1:1-19.

Matsumoto, R., 1983. Mineralogy and geochemistry of carbonate diagenesis of the Pliocene and Pleistocene hemipelagic mud on the
Blake Outer Ridge, Site 533, Leg 76. In Sheridan, R. E., Gradstein, F. M., et al., Init. Repts. DSDP, 76: Washington (U.S. Govt. Printing Office), 411-427.

Matsumoto, R., and Matsuhisa, Y., 1986. Chemistry, carbon oxygen and isotope ratios, and origin of deep-sea carbonate at Sites 438, 439, and 584: inner slope of the Japan Trench. In Kagami, H., Kang, D. E., et al., Init. Repts. DSDP, 87: Washington (U.S. Govt. Printing Office), 669-678.

Müller, P. S., and Suess, E., 1979. Productivity, sedimentation rate, and sedimentary organic carbon content in the oceans. Deep-Sea Res., 26:1347-1362.

Okada, H., 1980. Pebbles and carbonate nodules from Deep Sea Drilling Project Leg 56 cores. In Scientific Party, Init. Repts. DSDP, 56, 57(Pt. 2): Washington (U.S. Govt. Printing Office), 1089-1106.

Pederson, T. F., and Price, N. B., 1982. The geochemistry of manganese carbonate in Panama Basin sediments. Geochim. Cosmochim. Acta, 46:59-68.

Piper, D. Z., and Graef, P. A., 1974. Gold and rare-earth elements in sediments from the East Pacific Rise. Mar. Geol., 17:287-297.

Sayles, F. L., Ju, T. K., and Bowker, P. C., 1975., Chemistry of ferromanganoan sediment of the Bauer Deep. Geol. Soc. Am. Bull., 86: 1423-1431.

Shackleton, N. J., 1986. Paleogene stable isotope events. Palaeogeogr., Palaeoclimatol., Palaeocol., 57:91-102.

Shackleton, N. J., and Kennett, J., 1975. Paleotemperature history of the Cenozoic and the initiation of Antarctic glaciation; oxygen and carbon isotope anaklysis in DSDP Sites 277, 279, 281. In Kennett, J. P., Houtz, R. E., et al., Init. Repts. DSDP, 29: Washington (U.S. Govt. Printing Office), 743-755.

Sharma, T., and Clayton, R. N., 1965. Measurement of ${ }^{18} \mathrm{O} /{ }^{16} \mathrm{O}$ ratios of total oxygen from carbonates. Geochim. Cosmochim. Acta, 29: $1347-1354$.

Suess, E., 1979. Mineral phases in anoxic sediments by microbial decomposition of organic matter. Geochim. Cosmochim. Acta, 43: 339-352.

Tarutani, T., Clayton, R. N., and Mayeda, T. K., 1969. The effect of poilymorphism and magnesium substitution on oxygen isotope fractionation between calcium carbonates and water. Geochim. Cosmochim. Acta, 33:987-996.

von Rad, V., and Botz, R., 1986. Authigenic Fe-Mn carbonates in Cretaceous and Tertiary sediments of the continental rise off eastern North America, DSDP Site 603. In van Hinte, J., Wise, S., et al., Init. Repts. DSDP, 93: Washington (U.S. Govt. Printing Office), 1061-1078.

Wada, H., Niitsuma, N., Nagasawa, K., and Okada, H., 1982. Deepsea carbonate nodules from the Middle America Trench area off Mexico, Deep Sea Drilling Project Leg 66. In Watkins, J. S., Moore, J. C., et al., Init. Repts. DSDP, 66: Washington (U.S. Govt. Printing Office), 453-474.

Wada, H., and Okada, H., 1983. Nature and origin of deep-sea carbonate nodules collected from the Japan Trench. In Watkins, J. S., and Drake, C. L. (Eds.), Studies in Continental Margin Geology. AAPG Mem, 34:661-672.

Wedepohl, K. H., 1971. Geochemistry: New York (Holt, Rienhart, and Winston).

Date of initial receipt: 5 April 1988

Date of acceptance: 3 February 1989

Ms 105B-157 